\title{
Abstracts from the
}

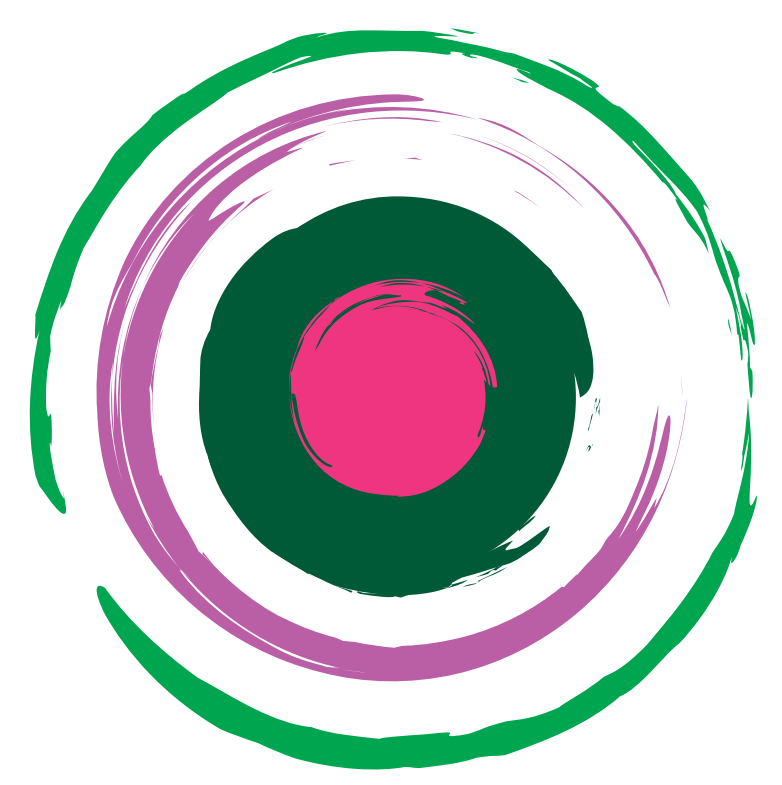

\section{World CONGRESS ON Huntington's Disease}

\author{
Sheraton Rio Hotel \& Resort \\ September I5-I8, 20I3 | Rio de Janeiro | Brazil
}
Francisco Cardoso
Ann Jones
Claudia Perandones
Raymund Roos
Cristina Sampaio

International Organising Committee:

Bernard Landwehrmeyer

\section{Local Organising Committee:}

Taíse Cadore

Francisco Cardoso

Monica Haddad

Denise Nicaretta 



\section{Editorial}

Dear Colleagues and Friends,

We would like to congratulate the organizers of the 2013 World Congress on Huntington's Disease (WCHD13), being held between 15-18 September in Rio de Janeiro, Brazil, on the development of an exciting congress program. This conference, a joint effort of the World Federation of Neurology (WFN) Research Group on Huntington's Disease and the International Huntington Association (IHA), provides an open forum for scientists, physicians, patients, and families to come together and share their experiences, unique understanding, and knowledge of HD. We believe that this congress will help further our collective goal of advancing the treatment of Huntington's Disease.

Such a wealth of promising research deserves an international forum, and as Editors-in-Chief, we are delighted to participate in the WCHD-2013 by publishing the scientific abstracts in the Journal of Huntington's Disease (http:// www.iospress.nl/journal-of-huntingtons-disease/), and by making these abstracts available online in an openaccess format. It is an honor for our new journal to play a critical role in promoting this important international conference.

The Journal of Huntington's Disease is dedicated to serving the HD community, and we hope that our publication of the WCHD-2013 abstracts is a useful and positive contribution to this important congress.

Blair R Leavitt, MDCM

University of British Columbia

Vancouver

\section{Editors-in-Chief}

Leslie M Thompson, PhD

University of California

Irvine 



\title{
Preface
}

\section{World Congress of Huntington's Disease in Rio de Janeiro, Brazil}

\author{
Francisco Cardoso MD $\mathrm{PhD}^{1}$, Monica Haddad $\mathrm{MD}^{2}$ \\ ${ }^{1}$ Chair of the Organizing Committee of the 2013 World Congress of Huntington's Disease; Professor of Neurology, \\ Neurology Service, Department of Internal Medicine, The Federal University of Minas Gerais, Belo Horizonte, \\ MG, Brazil \\ ${ }^{2}$ Vice-Chair of the Local Organizing Committee of the 2013 World Congress of Huntington's Disease; Neurologist, \\ Movement Disorders Unit, Neurology Division, Hospital das Clínicas, Sao Paulo University Medical School, \\ Sao Paulo, SP, Brazil
}

On behalf of the International and Local Organizing Committe of the 2013 World Congress of Huntington's Disease, we would like to salute the Huntington's Disease (HD) community on the occasion the World Congress takes place in Rio de Janeiro, Brazil.

It is true that 30 years after the discovery of its gene, HD remains a dreadful and devastating illness without a treatment that deters its relentless and inexorable progression. There are, however, reasons for hope. Arguably, this has been the most scientifically productive moment of the history of HD. In fact there is an outstanding number of published studies in the field. The average quality of both basic and clinical science publications is outstanding. The future scenario looks bright as well given the numerous ongoing investigations. Of note this is a truly interdisciplinary field where physicians, other health professionals and scientists of different backgrounds work together aiming to better understand and fight HD. One needs also to mention that the patients and their relatives play a meaningful part in driving the progress of care and science in HD.

The spirit of the 2013 World Congress of Huntington's Disease in Rio de Janeiro meeting is to celebrate the achievements of HD. A scientific program that has brought together speakers who are among the best professionals in the field will do this. They will share their experience, expertise and knowledge not only with an audience of professionals but also patients and relatives. In this regard, along the days of the World Congress there will be a sequence of talks specifically designed to bring cutting edge information to the non-professional audience.

The Rio de Janeiro meeting will also be the first time Latin America held a World Congress of Huntington's Disease. It will also mark the launching of the Enroll-HD study in South America. Given the utmost importance of this study to foster better care and research in the field of $\mathrm{HD}$, this is indeed a particularly joyful occasion for clinicians, other-health professionals, scientists and particularly patients living in this part of the world.

We cannot conclude without expressing our gratitude to the sponsors, the International Parkinson and Movement Disorders Society, Academia Brasileira de Neurologia and, in special, CHDI for the support that have made possible holding the 2013 World Congress of Huntington's Disease in Rio de Janeiro. 



\section{Abstracts}

\section{A COMPARISON OF THE IN VIVO NEUROCHEMICAL AND BEHAVIOURAL EFFECTS OF PRIDOPIDINE VS. ANTIDOPAMINERGIC COMPOUNDS USED IN HD}

Susanna Waters, Henrik Ponten, Clas Sonesson, Nicholas Waters

\begin{abstract}
.
Introduction: Drugs affecting monoamine transmission are frequently used in the clinical care of patients with Huntington's disease (HD). Tetrabenazine (TBZ), a monoamine depleting agent, is commonly used to alleviate chorea. Antipsychotic compounds, ie dopamine (DA) D2 antagonists, are also used, mainly to reduce chorea and behavioural disturbances. Both TBZ and D2 antagonists are prone to antidopaminergic side effects such as parkinsonism. Pridopidine is a dopaminergic stabilizer under clinical evaluation for improving motor impairment in patients with HD. Pridopidine modulates DA transmission but has also been proposed to affect synaptic NMDA receptor mediated transmission in the prefrontal cortex. Aims To compare the in vivo neurochemical and behavioural effects of pridopidine vs anti-dopaminergic drugs used in HD.

Methods: Dose-response experiments were performed with pridopidine, haloperidol, olanzapine, risperidone and TBZ dosed sc to male Sprague-Dawley rats. Locomotor activity was assessed for $60^{\prime}$. Post mortem brain tissues were subject to neurochemical analysis and mRNA assessment. In vivo microdialysis, assessing monoamines in the frontal cortex and striatum, was also performed.

Results: All compounds produced increased DOPAC, reflecting reduced $\mathrm{D} 2$ receptor tone, while TBZ but not the other test drugs markedly reduced tissue monoamines. All compounds, except pridopidine, reduced spontaneous locomotor activity. Microdialysis showed increased cortical monoamine release for several agents, including pridopidine, but reductions with TBZ. All compounds increased striatal Arc gene expression. In addition, pridopidine significantly increased Arc gene expression in the frontal cortex. This was not seen with the other compounds.

Conclusions: These DA signalling affecting compounds share neurochemical features reflecting reduced D2 receptor tone, but differ in their downstream effects especially on cortical neurotransmission. Pridopidine displays a unique combination of increased cortical Arc, suggesting enhanced synaptic transmission, and no inhibition of spontaneous locomotor activity. These two features may be key to the improved motor function and lack of psychomotor depressant side effects observed in clinical trials with pridopidine in patients with HD. This differentiates pridopidine from antidopaminergic compounds currently used in HD.
\end{abstract}

\section{A DRUG-DRUG INTERACTION STUDY OF PRIDOPIDINE, A NEW DRUG FOR TREATMENT OF HUNTINGTON'S DISEASE, AND METOPROLOL, A CYTOCHROME P450 2D6 SUBSTRATE}

Laura Rabinovich-Guilatt, Ofer Spiegelstein, Anna Wickenberg, Merav Bassan

\section{Abstract.}

Background: Pridopidine, a new drug for the symptomatic treatment of Huntington's disease (HD), is currently under development by Teva Pharmaceuticals. In vitro data suggests that pridopidine is a substrate of the cytochrome P450 enzyme CYP2D6 (CYP2D6), and that it is also an inhibitor of this enzyme.

Objective: The primary objective of this clinical study was to evaluate the impact of pridopidine on CYP2D6 activity in vivo, by co-administering the CYP2D6 probe substrate metoprolol. Methods This was an open-label, single sequence, crossover study in 22 healthy male and female volunteers with the CYP2D6-extensive metabolizer (EM) genotype. Metoprolol (100 mg single oral dose) was administered before and after 7 days of pridopidine (45 mg twice daily oral dosing).

Results: Pridopidine administration resulted in approximately 3.5-fold higher Cmax and approximately 6.5-fold higher AUC of metoprolol compared to baseline. The $t 1 / 2$ of metoprolol increased from 3.7 hours to 5.6 hours, whereas the time to maximum metoprolol concentration in plasma (tmax) remained unchanged.

Conclusion: These findings indicate that pridopidine is a strong inhibitor of the CYP2D6 enzyme and increased plasma concentrations of co-administered CYP2D6 substrate drugs can be expected. Several medications used for the treatment of HD are known to be metabolized by CYP2D6, therefore adequate clinical monitoring should be exercised. These findings are in agreement with the time-dependent pharmacokinetics seen for pridopidine in CYP2D6 EM subjects, but absent in poor metabolizer (PM) subjects. Due to auto-inhibition of CYP2D6 in EMs the exposure in both populations approach each other after multiple doses.

\section{A MULTIDISCIPLINARY INTENSIVE REHABILITATION PROGRAM FOR PATIENTS WITH HUNTINGTON'S DISEASE - EFFECTS ON COGNITIVE FUNCTION}

Marleen van Walsem, Anu Piira, Geir Mikalsen, Kjell Haavik Nilsen, Synnøve Fønnebø Knutsen, Jan C Frich

Abstract.
Introduction: Studies suggest that patients with Huntington's
disease (HD) benefit from intensive rehabilitation, but there is 
limited evidence as to the effect on cognitive function, and if achieved results are sustained over longer time. Objectives: We assessed the effect of an intensive, one-year multidisciplinary rehabilitation program in patients with HD, using a broad neuropsychological screening battery.

Methods: 37 patients, aged $18+$ years enrolled in a 1-year program consisting of $3 \times 3$ weeks intensive inpatient rehabilitation and $1 \times 5$ days evaluation stay at two rehabilitation centers. Inclusion criteria: known genetic diagnosis of HD, Schoulson \& Fahn stage I-III, no diagnosis of severe psychiatric illness, no apparent severe general cognitive impairment at the time of first admission, and full/ mostly full independence in Activities of Daily Living. Mean $\mathrm{TFC}=8.9$, mean age: 52.4 years; mean symptom duration: 7.2 years; mean UHDRS motor score 36.6, and mean UHDRS behavioral score 9.2. Mean MMSE score: 25.4. The program included 8 hours of daily physical exercise, group discussions, teaching sessions, and social activities. Besides the UHDRS Cognitive assessment, neuropsychological measures assessing verbal function, verbal learning and memory, additional measures for psychomotor speed and executive function were used.

Results: A total of 31 patients (84\%) completed the one year program and were available for neuropsychological testing at the evaluation stay. Eight of 16 measures showed a slight decline, with only one measure showing a significant decline with a medium effect size (Symbol Digit Modality Test: mean change $-2.9 ; p=0.027, r=0.42)$ and one measure showing a medium effect size with a $\mathrm{p}$ value $<0.10$ (CVLT-II Long term memory: mean change $-0.9 ; p=0.07, r=0.34)$. The remaining eight measures showed positive, but non-significant changes. Effects of this program on motor function, ADL function, quality of life, symptoms of depression and anxiety, MMSE and BMI are reported elsewhere.

Conclusion: The results suggest a non-significant positive effect on patients with early and mid stage HD on measures of cognitive function in a one-year long multidisciplinary intensive rehabilitation program and is well-tolerated. However, two medium effect sizes were found for a psychomotor task and for a measure for long-term verbal memory.

\section{A MULTIDISCIPLINARY INTENSIVE REHABILITATION PROGRAM FOR PATIENTS WITH HUNTINGTON'S DISEASE - RESULTS AFTER 2-YEARS FOLLOW-UP}

Anu Piira, Marleen van Walsem, Geir Mikalsen, Lars Øie, Jan C. Frich, Synnøve Fønnebø Knutsen

\footnotetext{
Abstract.

Introduction: Studies suggest that patients with Huntington's disease (HD) benefit from intensive rehabilitation, but there is limited evidence on whether achieved results are sustained over a longer time period.

Objectives: To assess the effects of an intensive, multidisciplinary two year long rehabilitation program in patients with HD.

Methods: Ten patients, aged $18+$ years enrolled in a 2-year long program with $6 \times 3$ weeks intensive rehabilitation and 2 $\times 5$ days evaluation stay. Inclusion criteria: Schoulson $\&$ Fahn
}

stage I-III, no severe psychiatric illness, none/slight reduced cognitive function, and full/mostly full independence in Activities of Daily Living. Mean (SD) $\mathrm{TFC}=8.7$, mean age: 50.0 years; mean symptom duration: 6.6 years; mean UHDRS motor score 47.4, and mean UHDRS behavioral score 7.4. Mean MMSE score: 23.5. The program included 8 hours of daily physical exercise, group discussions, teaching sessions, and social activities.

Results: Six out of ten patients were able to complete the entire rehabilitation program. Slight, but not significant, changes were observed in gait and balance from baseline to 24 months evaluation: 6 min walking test: $-16.3 \mathrm{~m} ; 10$ meter walk test: $+3.1 \mathrm{sec}$; timed-up-and-go test: $+3.6 \mathrm{sec}$; Bergs balance scale: -2.2 points; and Activities-spesific Balance Confidence $(A B C)$ scale -8.7 points. No change in ADLfunction (Barthel index): -0.2 points. No significant changes in MMSE +1.3, or UHDRS cognitive assessments were found: Stroop color: -0.3 , Stroop word: +0.3 , Stoop int:-5.5, FAS: -1.0 , SDMT: -5 , Self-reported health-related quality of life (SF-36): improvement in mental health: +10.3 , and in physical health: +13.0. HADS and BMI both showed non-significant improvement of -4.0 points and +2.4 units, respectively.

Conclusion: Patients with early and mid stage HD showed a slight non-significant decline in motor and cognitive function during, a two-year long multidisciplinary intensive rehabilitation program. Positive effects were sustained in quality of life, BMI and reduced symptoms of anxiety and depression, although changes were not statistically significant. Our findings suggest that participation in an intensive rehabilitation program is well tolerated among HD patients. Interpretation of results should be done carefully due to small sample size.

\section{A PLATFORM FOR STUDIES OF HUNTINGTON'S DISEASE AND OTHER NEURODEGENERATIVE DISORDERS ON THE BASIS OF INDUCED PLURIPOTENT STEM CELLS}

Maria A. Lagarkova, Sergey L. Kiselev, Igor. A. Grivennikov, Olga S. Lebedeva, Sergey A. Klyushnikov, Ilya V. Chestkov, Ekaterina Yu. Fedotova, Maria N. Zakharova, Sergey N. Illarioshkin

\section{Abstract.}

Introduction: A new technology of genetic reprogramming allows obtaining induced pluripotent stem cells (iPSC) from fibroblasts and other somatic cells. These iPSC can grow in culture and differentiate in any cell types, including neurons. Methods: We obtained, with the use of lentivirus transfection, iPSC from primary fibroblasts biopsied from three patients with HD (42-46 copies of the CAG repeats in the mutant allele), three patients with genetic forms of Parkinson's diseases (PD) and two patients with SOD1-associated amyotrophic lateral sclerosis (ALS). In these experiments we used a multielectrode arrays (MEA) for precise noninvasive long-term neural network stimulation and measurement of the electric signals, as well as for structural/functional imaging of metabolic ionic currents using confocal laser scanning microscopy. 
Results: The efficiency of reprogramming was approximately $0.2 \%$. Derivatives of all the three embryo layers were shown to be formed as a result of spontaneous iPSC differentiation. Thereafter, iPSC from HD, PD and ALS were differentiated, respectively, into GABA-ergic, dopaminergic and motor neurons, as confirmed by staining with $\mathrm{TH}$ and other specific reactions.

Conclusion: Our MEA-based system represents a unique cell platform aimed at discoveries of molecular mechanisms of neurodegenerative disorders and high-throughput search for novel neuroprotective drugs. The study was supported by a grant from Russian Foundation for Basic Research (RFBR).

\section{A PROSPECTIVE PILOT TRIAL FOR PALLIDAL DEEP BRAIN STIMULATION (DBS) IN HUNTINGTON'S DISEASE (HD) - 12 MONTH FOLLOW-UP OF CHOREA, DYSTONIA, HYPOKINESIA, COGNITION, MOOD AND QUALITY OF LIFE}

Jan Vesper, Lars Wojtecki, Stefan J Groiss, Saskia Elben, Stefano Ferrea, Alfons Schnitzler

\begin{abstract}
:
Introduction: DBS in HD was evaluated in a prospective pilot trial. Primary endpoint results after a preceding randomised cross-over period each for 6 weeks of either more internal (GPI) or external (GPE) parts of the pallidum (primary endpoint) and after 3 months of best contact stimulation showed significant suppression of chorea. Long-term effects on cognition, mood and quality of will now be reported after 12 months follow-up as secondary study endpoint.

Methods: 6 HD patients (2 westphal variants) were examined. The following outcome parameters were assessed at 12 months follow-up and were compared with preop baseline: UHDRS, UHDRS chorea subscore, Burke-Fahn-MarsdenDystonia-Rating-Scale (BFMDRS), Unified-Parkinson'sDisease-Rating-Scale (UPDRS-III), cognition (Mattis), depression (Becks-Depression-Inventary, BDI), Activity of Daily-Living (HD-ADL) and quality of life SF-36 score.

Results: UDHRS motor score and changed from 55 (SD18) to 53 (SD 25), n.s. Excluding westphal patients from analysis, UHDRS motor score change was significant (47 (SD 15) to 36 (SD 12), $p<0.05, n=4)$. The same comprised for the chorea subscore (9 (SD 8) to 4 (SD 4), $p<0.1, n=6$ and 13 (SD 4) to 6 (SD2), $p=0.05$, for $n=4$ respectively). BFMDRS und UPRDS results were heterogeneous: scores generally worsened over the whole group but improved n.s in the nonwestphal group. BDI, Mattis and HD-ADL were stable. SF-36 vitality and mental health improved significantly $(p<0.05)$. Three serious adverse events occurred: one pneumonia/sepsis, one traumatic subdural haematoma with non-convulsive status epilepticus and one death due to aspiration pneumonia following epistaxis. None of the SAE were judged as treatment related.

Conclusion: This is the first prospective pilot trial reporting DBS in HD. Long-term effects after 12 months follow-up show stable suppression of chorea, no detoriation in mood and cognition and significant improvement in SF-36 vitality and mental health quality of life assessments. Distinct motor effects e.g. on dystonia and functional relevance of DBS on
\end{abstract}

quality of life despite disease related adverse events should be addressed in a larger multi-center and sham-stimulationcontrolled trial.

\section{ABERRANT SPLICING OF HTT GENERATES THE PATHOGENIC EXON 1 PROTEIN IN HUNTINGTON DISEASE}

Kirupa Sathasivam, Andreas Neueder, Theresa A. Gipson, Christian Landles, Agnesska C. Benjamin, Marie K. Bondulich, Donna L. Smith, Richard L. Faull, Raymund A. Roos, David Howland, Peter J. Detloff, David E. Housman, Gillian P. Bates

Abstract. Huntington's disease is caused by a polyglutamine expansion close to the $\mathrm{N}$-terminus of the huntingtin protein and the formation of huntingtin fragments has been implicated as an essential step in the molecular pathogenesis of this disorder. However, the mechanism responsible for generating small highly pathogenic fragments has not been identified despite an intense search over the past ten years. Here we show that the CAG repeat length-dependent aberrant splicing of the huntingtin gene results in a short polyadenylated mRNA that is translated into an exon 1 HTT protein. This discovery provides a novel mechanistic basis for the initiation of the molecular pathogenesis of Huntington's disease. Our findings have considerable implications for the development of therapeutics that try to lower the levels of mutant huntingtin. Our results have also opened up new therapeutic avenues for consideration. Strategies that target mis-splicing are in active development for many other disorders and these approaches can now be applied to Huntington's disease.

\section{ABNORMAL BRAIN STRUCTURE AND FUNCTION IN CHILDREN WITH GENE EXPANSION FOR HUNTINGTON'S DISEASE: EVIDENCE FOR ABNORMAL BRAIN DEVELOPMENT}

Peggy Nopoulos, Jessica Lee, Vincent Magnotta, Eric Epping, Kathy Mathews, Jeffrey Dawson

\section{Abstract.}

Background: The pathophysiology of Huntington's Disease (HD) is classically conceptualized as a neurodegeneration of the striatum. However, growing evidence has supported the notion that abnormal brain development may be a vital part of the pathophysiology of this, and possibly other degenerative brain disorders. The KidsHD program is designed to evaluate children (ages 6-18 years) who are at risk for HD. This provides the opportunity to evaluate brain structure and function in those with expanded genes, but decades from onset of disease.

Methods: Children ages 6-18 years who have a parent with HD are enrolled in the study only if they are currently not manifesting symptoms of the disease. Brain structure is evaluated using Magnetic Resonance Imaging (MRI) and brain function in quantified by cognitive and motor 
assessments. For research purposes, each participant is genotyped. Those that have CAG repeats $<39$ are gene nonexpanded (GNE, $n=44)$ and those with CAG repeats 40 are gene-expanded (GE, $n=39$ ). In addition, healthy children (HC) from families with no HD were enrolled $(n=104)$. MRI was used to obtain structural data (volumes). A battery of cognitive tests was administered and the Physical and Neurological Examination of Subtle Signs quantified motor function.

Results: GE participants had substantially smaller total brain tissue volumes compared to both HC and GNE samples. After controlling for the smaller brain, the striatum was substantially reduced in volume. In addition, the thalamus was somewhat enlarged. The GNE group had no significant differences compared to the controls in any measure. Children with CAG repeats in the range of 40-44 repeats were doing well in terms of motor and cognitive symptoms, despite the abnormal brain structure. Children with CAG repeats 45 and above showed significant deficits in a wide range of cognitive and motor skills.

Conclusion: In this sample of children who are geneexpanded, yet greater than an estimated 30 years from onset, brain structure, is substantially altered compared to controls. For the children in the 'low CAG repeat range' (40-44), they appeared to have adequate functional compensation as this group had normal motor and cognitive skills (with some areas being superior to controls). Those is longer CAG repeats had both structural and functional brain deficits. This supports the notion that aberrant brain development may play an important role in the pathophysiology of the disease.

\section{ABNORMAL IRON CONCENTRATION IN HUNTINGTON DISEASE BRAIN}

Michael Dayan, Ferdinando Squitieri, Umberto Sabatini

\footnotetext{
Abstract.

Introduction: Iron accumulation in basal ganglia is associated with striatal neuron loss in Huntington Disease (HD). T*2weighted imaging is a useful measure to study iron distribution in the brain since magnetic susceptibility maps correlate with chemically-determined iron concentration (Langkammer, 2012). Increased levels of iron were detected in several brain areas including Caudate, Putamen, Globus Pallidus and cortex in both symptomatic and premanifest subjects (Bartzokis, 1999-07; Sanchez-Castaneda, 2012; Dumas, 2012; Rosas 2012). However, whether the iron accumulation is related to the clinical stage or to the gray matter loss, is far to be understood. Objectives: To explore iron abnormalities in HD clinical subgroups and their relationship with gray matter atrophy.

Methods: 77 HD subjects (CAG repeats $>39$ ) and 73 matched controls underwent $\mathrm{T} * 2$ relaxometry (multiecho EPI sequence) and 3D T1-weighted imaging (MDEFT sequence) on a 3T Siemens scanner. Subject groups included: 29 premanifest subjects $(n=20$, far-from-onset individuals (preHD) with UHDRS motor score $<5$ and 9 near-to-onset individuals with soft signs (preHD-SS)) and 48 symptomatic subjects (HD). Family wise error (FWE) rate correction at $p<0.05$.
}

Results: a) Iron starts to accumulate in Caudate since the presymptomatic life stage and gradually and progressively decreases with disease progression; iron concentration, instead, increases in Putamen and Globus Pallidus. b) As expected, basal ganglia volume decreases. Such decrease starts in the Caudate in PreHD, later progressing to the Putamen and Globus Pallidus. c) In cortex we observed a progressive iron decrease starting in Parieto-Occipital areas in PreHD-SS and extending to Temporal, Cingulate and larger Parieto-Temporal areas, in patients. d) There is also a progressive cortical volume loss; starting in posterior Frontal areas in premanifest subjects, with the overall involvement of the cortex, but relative preservation of anterior Frontal regions in patients.

Conclusions: In basal ganglia, we observe a progressive iron increase and volume decrease since the premanifest life stage, starting from Caudate, and then extending to Putamen and Globus Pallidus. There is a significant correlation between iron accumulation, volume loss and disease progression. Conversely, iron content decreases in brain cortex and is associated with the volume loss in posterior areas.

\section{ACTION-HD: A RANDOMIZED, DOUBLE- BLIND, PLACEBO-CONTROLLED PROSPECTIVE CROSSOVER TRIAL INVESTIGATING THE EFFICACY AND SAFETY OF BUPROPION IN HUNTINGTON'S DISEASE (HD)}

Harald Gelderblom, Wilhelm Fischer, Tim McLean, Ralf Reilmann, Carsten Saft, Sigurd Süssmuth, Bernhard Landwehrmeyer, Eike Jakob Spruth, Josef Priller
Abstract.
Background: Apathy is a common behavioural symptom in $\mathrm{HD}$, repeatedly ranked as one of the most pressing problems by HD specialists. It is defined as the primary absence of motivation, lack of initiative and drive, as well as emotional blunting. Apathy has been related to dysfunctions of frontal lobe areas receiving dopaminergic projections thought to be involved in reward and motivation. Effects of dopamine (DA) are regulated by norepinephrine (NE) reuptake in the frontal cortex, which largely lacks DA transporters. Bupropion blocks NE and DA reuptake, hereby potentially increasing DA neurotransmission in areas particularly relevant for the generation of apathy.
Aims: To investigate the efficacy and safety of bupropion in the treatment of apathy in HD.
Methods: In a crossover design, apathetic HD mutation carriers are randomized to receive either bupropion $150 / 300 \mathrm{mg}$ or placebo daily. The primary outcome parameter is a significant change of the the AES (apathy evaluation scale) score as judged by a family member after ten weeks of treatment. Secondary outcome parameters are changes of the AES scores determined by patient or physician, changes of psychiatric, cognitive and motor symptoms, in activities of daily living and caregiver distress. Changes of DA-mediated ventral striatal and ventromedial prefrontal activation will be investigated in response to a reward paradigm as quantified by functional magnetic resonance imaging. 
Results: Action-HD has been actively recruiting patients since May 2012. Recruitment will be finalized in August 2013. Depression and co-medication with antipsychotics were rigorously considered as exclusion criteria. A gambling paradigm was established, which reproducibly induces DAmediated activation during reward anticipation. It was successfully tested in 50 healthy individuals, adapted to chorea, and corrected for changes in vasoreactivity due to age and medication.

Conclusion: Action-HD is the first multi-centre investigatorinitiated trial dealing with the symptomatic treatment of apathy in HD. Results of this trial may help to develop an evidence-based therapy for one of the most debilitating neuropsychiatric symptoms of HD. In addition, this trial may help to define standards for conducting future trials for neuropsychiatric symptoms of HD. Challenges associated with the development and conduct of Action-HD will be discussed. Funding: This trial is supported by a grant from HSG/HSC, NeuroCure (DFG) and EHDN.

\section{ACUTE-PHASE RESPONSE IN HUNTINGTON'S DISEASE}

Anna A.M. Hubers, Jos A. Bouwens, Erik van Duijn, Christa M. Cobbaert, Raymund A.C. Roos, Rose C. van der Mast, Erik J. Giltay

\footnotetext{
Abstract.

Background: There is increasing evidence that the immune system may be involved in the pathogenesis of Huntington's disease (HD). Increased activation of components of the innate and adaptive immune systems has been found in the central nervous system and peripheral tissues of HD patients. Objective: This study aims to investigate serum levels of C-reactive protein (CRP) and albumin, as positive and negative markers of the acute-phase response respectively, in relation to clinical, neuropsychiatric and cognitive characteristics, as well as the use of psychotropics, in HD mutation carriers.

Method: At baseline, the study cohort consisted of $122 \mathrm{HD}$ mutation carriers and 42 controls. After two years follow-up, the high sensitivity CRP and albumin levels and clinical, neuropsychiatric, and cognitive characteristics were reassessed in $85 \mathrm{HD}$ mutation carriers and 32 controls. Associations between acute-phase proteins and clinical, neuropsychiatric, and cognitive characteristics at both time points were analyzed using multivariate linear and logistic regression analysis.

Results: At baseline, $44 \%$ of the mutation carriers were male with a mean $( \pm \mathrm{SD})$ age of $49.2( \pm 11.5)$ years. Significant associations were found between acute-phase proteins and Total Functioning Capacity (TFC) score, apathy, cognitive impairment and the use of antipsychotics. However, all significant associations disappeared after additionally adjusting for antipsychotic use. High sensitivity CRP levels were highest and albumin levels were lowest in mutation carriers who used antipsychotics at both time points, compared with mutation carriers who did not use antipsychotics.

Conclusion: The associations found between acute-phase proteins and TFC score, apathy, and cognitive impairment were influenced by the use of antipsychotics. HD mutation carriers who receive antipsychotics may be susceptible to having an elevated acute-phase response.
}

\section{ALTERATIONS IN THE MORPHOLOGY OF STERNOMASTOID AND SOLEOUS MUSCLES IN BACHD MICE}

Priscila Aparecida Costa Valadão, Bárbara Campos de Aragão, Rubens Garcias, Carla Oliveira Veriano, Matheus Proença Simão Magalhães Gomes, Fabíola M. Ribeiro, Cristina Guatimosim

Abstract. Huntington's disease (HD) is a progressive, debilitating, fatal neurological disease caused by a CAG trinucleotide repetition that leads to a polyglutamine expansion in the huntingtin (htt) protein. Its symptoms include chorea, cognitive disturbances and progressive motor decline starting with motor impairment of the muscles of the face and then the muscles of the trunk and limbs. The aim of this study is to evaluate the morphological changes of different muscular groups such as sternomastoid (ETM), located on the neck and Soleus (SOL), a lower limb muscle, from BACHD mice, a transgenic model for HD. Briefly, ETM and SOL skeletal muscles from at least three pairs of three-month-old-male BACHD and WT animals were dissected, fixed in glutaraldehyde 4\%, dehydrated in an alcohol ascendant series, embedded and included in Glycol methacrylate. Cross sections $(5 \mu \mathrm{m})$ were cut and stained with toluidine blue. We analyzed the distribution of muscle fibers in fixed intervals of area and perimeter. We applied the Kolmogorov-Smirnov statistical test in a graph of cumulative frequency where we evaluated the area and perimeter of muscle fibers. We analyzed 7475 muscle fibers in ETM and 1585 muscle fibers in SOL for each genotype. Our results shows that in ETM muscle, more muscle fibers were located at higher intervals of area and perimeter in BACHD compared to WT. The cumulative frequency analysis showed that the area in ETM from BACHD mice presented about $80 \%$ of the fibers with more than $4000 \mu \mathrm{m} 2$ whereas in WT mice, the same percentage of the fibers measured $2000 \mu \mathrm{m} 2(p<0,0001)$. Regarding the perimeter, we observed that $80 \%$ of the muscle fibers from WT measured $160 \mu \mathrm{m}$ whereas in BACHD the perimeter measured 250 $\mu \mathrm{m}(p<0,0001)$. By contrast, we did not observe any difference in the distribution of muscle fibers in the SOL, a slow twitching muscle, when comparing the genotypes. Our results so far suggest that distinct muscular groups are affected differently in BACHD mice model at the age of three months. We are currently analyzing other muscle groups in order to investigate if slow twitching muscle fibers are more susceptible then fast twitching in BACHD mice.This work was supported by CAPES, CNPq and FAPEMIG.

\section{AN EXPLORATION OF THE ABNORMALITIES OF HIPPOCAMPAL NEUROGENESIS AND PROLIFERATION IN THE R6/1 MOUSE MODEL OF HD}

\section{Jina Pakpoor, Roger Barker, Faye Begeti}

\section{Abstract.}

Introduction: Huntington's disease (HD) is an autosomal dominant neurodegenerative condition characterized by movement disorders, psychiatric disturbance, and cognitive decline. Previous work has demonstrated a reduction in adult 
hippocampal neurogenesis in transgenic mouse models, known to be important in hippocampal-dependent cognition. It is widely accepted that neurogenesis occurs in two distinct areas of the adult mammalian brain; the subventricular zone (SVZ) and the subgranular zone (SGZ) of the dentate gyrus (DG) in the hippocampus Objectives To examine the abnormalities of hippocampal neurogensis and proliferation in the R6/1 mouse model of HD and explore the role of stem/ neural precursor cells in abnormalities of adult hippocampal neurogenesis.

Methods: In 16 week old R6/1 mouse four commonly used markers were used to stain the dentate gyrus. The stained cells were subsequently quantified. Mouse anti-rat Nestin and rabbit anti-sox 2 were used to identify stem/neural precursor cells; rabbit anti-Ki67 was used to identify proliferating cells; and goat anti-DCX to identify neurogenesis.

Results: Proliferating cells and immature neurons were significantly reduced in the dentate gyrus DG of R6/1 mice, compared to WT littermates [Ki67: $t=6.23, P<0.001$, DCX: $t=7.46, P<0.001]$. The number of nestin + processes transversing the DG was signifcantly increased in R6/1 mice $[t=-4.182, p=0.002]$, compared to WT. There were no differences between the number of Sox $2+$ cells in HD and WT mice.

Conclusions: This study shows that in addition to the welldescribed reduction in proliferation and immature neurons in the R6/1 HD model, there is a robust increase in nestin+ cells indicating that the deficit in adult hippocampal neurogenesis occurs at the neural stem/progenitor cell. Despite a large increase in nestin + processes, the number of sox $2+$ cells was unchanged between genotypes, which could be explained by an increase in astrogliosis in the DG. It is unclear whether these quiescent cells could be somehow manipulated in order to restore the neurogenesis impairment in HD mouse models. However, given that the increase in nestin+ cells was consistently observed in both cohorts of R6/1 mice examined in this study as well as in the more progressive R6/2 mouse model (Clelland et al. unpublished data), changes in this marker may be useful as a new outcome parameter to assess the efficacy of treatments aiming to restore the neurogenesis deficit in HD mice.

\section{AN INTEGRATED ANALYSIS OF THE HDHQ150/150 KNOCK-IN MOUSE MODEL OF HUNTINGTON'S DISEASE BY BEHAVIOR, IN VIVO MRI AND HISTOLOGY}

Ivan Rattray, Edward Smith, Thomas Walker, Richard Gale, Gillian Bates, Michel Modo

Abstract. The HdhQ150/Q150 knock-in mouse model of Huntington's disease (HD) carries an expanded polyglutamine sequence in the mouse huntingtin homologue. This results in the eventual development of HD-like pathology at a comparably slower rate than the more commonly used transgenic mouse models. Using a combination of behavioral assessments, MRI and histology, this study provides a longitudinal, interdisciplinary analysis of the progressive pathology exhibited by this mouse line with a particular focus on the early stages of disease progression. Both male and female, wild-type and HdhQ150/Q150 mice were used. To probe functional abnormalities, a detailed battery of behavioral measures was applied including rotarod, grip strength, open field, olfactory discrimination, social interaction, cued and contextual fear conditioning, as well as learning in a swimming T-maze. These tests were coupled with longitudinal in vivo MRI to monitor regional brain atrophy and $\mathrm{T} 2$ relaxivity (reflective of tissue composition) at $2,4,6,9,12,18$ and 22 months of age, whereupon the mice were culled. Preliminary analyses indicate progressive deficits in behavioral measures can be detected as early as 4 months of age. Moreover, age-related loss of volume in several key brain regions was a robust finding in this mouse line. High resolution ex vivo MRI was conducted prior to post-mortem histological analysis of neuropathology to further support these in vivo measures. Correlative analyses will ultimately provide links between behavioral phenomena and regional brain abnormalities determined through MRI and histology. This continuing description of the HdhQ150/Q150 knock-in mouse model will provide crucial information on the progression of HD-like pathology, as well as to what extent functional deficits are associated with neuropathological changes in the these mice. Support: Medical Research Council; CHDI Foundation.

\section{AN INTEGRATED ANALYSIS OF THE R6/1 TRANSGENIC MOUSE MODEL OF HUNTINGTON'S DISEASE BY BEHAVIOR, IN VIVO MRI AND HISTOLOGY}

Ivan Rattray, Edward Smith, William R. Crum, Thomas A. Walker, Richard Gale, Gillian Bates, Michel Modo

Abstract. The R6/1 transgenic mouse model expresses a delayed phenotypic onset compared to the more commonly used R6/2 mouse model of Huntington's disease (HD). Using a combination of serial behavioral assessments, in vivo MRI and post-mortem histology, this study provides a longitudinal, interdisciplinary analysis of the progressive pathology exhibited by this mouse line. Both male and female, wild-type and R6/1 mice were used. To probe functional abnormalities, a detailed battery of behavioral measures was applied, including rotarod, grip strength, open field, olfactory discrimination, social interaction, cued and contextual fear conditioning and learning in a swimming T-maze. These tests were coupled with longitudinal in vivo MRI to monitor regional brain atrophy and $\mathrm{T} 2$ relaxivity (reflective of tissue composition) at both 9 and 17 week of age. Deficits in behavioral measures developed with age, alongside a reduction of regional brain volumes in the R6/1s. Highresolution ex vivo MRI was conducted prior to post-mortem histological analysis to provide a more detailed description of neuroanatomical changes. Application of an automated image analysis method, Tensor Based Morphometry, on both the in vivo and ex vivo images provided a greater degree of accuracy and sensitivity to brain regional volume studies. We detected the accumulation of mutant huntingtin protein, as well as neuronal cell loss, in the brains of R6/1 mice at 19 weeks of age. This description of the R6/1 mouse line provides crucial information on the progression of HD-like pathology, as well as to what extent functional deficits are associated with 
neuropathological changes in this model. Support: Medical Research Council; CHDI Foundation.

\section{ANALYSIS OF HUNTINGTIN PROTEIN FRAGMENTS IN POST MORTEM HUMAN HUNTINGTON'S DISEASE BRAIN TISSUE}

Menno Schut, Jocelyn Bullock, Stefano Patassini, Eric Kim, Henry Waldvogel, Barry Pepers, Johan den Dunnen, Gert-Jan B. van Ommen, Richard Faull, Willeke van Roon-Mom

\begin{abstract}
.
Introduction: The exact mechanism of HD pathogenesis remains elusive, but it is thought that creation of an $\mathrm{N}$-terminal fragment by proteolytic cleavage of the mutant huntingtin protein is an important step. For this reason, presence of $\mathrm{N}$-terminal (m)Htt fragments in human brain tissue is a topic of interest. However, studies involving htt cleavage fragments in human brain tissue could be complicated by non-disease specific degradation of the htt protein during post-mortem delay.

Methods: To elucidate effects of post-mortem delay, we have used human HD caudate nucleus tissue and non-HD human temporal lobe tissue with low initial post-mortem delays (3 and 1 hours resp). To mimic post-mortem delay, specimens were brought to room-temperature and every 2 hours samples were taken for a minimum of eight hours. We also analyzed $\mathrm{N}$-terminal $(\mathrm{m}) \mathrm{htt}$ fragments in sensory/motor cortex and caudate nucleus from nine different HD and control subjects. Analysis was performed by Western-blotting using an antibody that recognizes the first 17 amino acids.

Results and Conclusion: Effects of post-mortem delay on the appearance of $\mathrm{N}$-terminal $(\mathrm{m}) \mathrm{Htt}$ fragments are only moderate. For the non-HD temporal lobe tissue, we found that the majority of fragments did not change between time points, apart from fragments at $52 \mathrm{kD}$ and $70 \mathrm{kD}$ which increased over time. For the HD caudate nucleus specimen, we found that several htt fragments between 80 to $100 \mathrm{kD}$ decreased over time. Within our 9vs9 cohort, we did not observe striking differences between HD and controls for the sensory/motor cortex. For the HD caudate nucleus however, we observed an increase in $(\mathrm{m}) \mathrm{htt}$ protein fragments compared to the control samples. This suggests that there is a regional variation in $(\mathrm{m})$ htt protein fragmentation in the human brain which may be related to pathogenesis.
\end{abstract}

\section{APATHY AND INFORMATION-PROCESSING IN HUNTINGTON'S DISEASE: INSIGHTS FROM EYE MOVEMENT}

\section{Judith Bek, David Craufurd}

\section{Abstract}

Background: Apathy is common in Huntington's disease (HD) and impairs quality of life for patients and families. Apathy predicts cognitive decline in other neurological conditions and may have distinct cognitive correlates in HD; clarifying the relationship between apathy and cognition could thus aid earlier detection of change and prediction of functional decline. In studies of older adults and schizophrenia, apathy has been associated with abnormal eye movement in exploratory information-processing. Also, while healthy adults show increased eye movements to emotive stimuli, this pattern is not found in Parkinson's disease, in which apathy is common. HD gene carriers show eye movement abnormalities which correlate with disease progression and brain volume changes. Based on these findings, eye movements might also reveal affective and cognitive changes, but previous research on these symptoms in relation to oculomotor change in HD is limited. We examined eye movement in relation to apathy and cognition, initially through analysis of EHDN Registry data and secondly through experimental eye-tracking work.

Objectives: We aimed to (1) identify relationships among apathy, eye movement and cognition using Registry data, and (2) examine the effects of apathy on eye movement during motivationally-relevant information-processing.

Methods: Oculomotor scores, apathy ratings and cognitive test scores from 2873 Registry participants were analysed. Partial correlations were calculated between oculomotor and apathy measures, oculomotor and cognitive measures, and apathy and cognitive measures, controlling for disease burden, UHDRS motor score and age. Results. Apathy ratings correlated significantly with saccade initiation and velocity, and with all cognitive measures (all $p<0.001$ ). A number of correlations were also found between cognitive and oculomotor scores, but few remained when controlling for apathy.

Conclusions: Analysis of the Registry data indicates that apathy is associated with both oculomotor and cognitive performance, and may mediate the relationship between cognitive and oculomotor scores. These relationships require further investigation, with more objective measurement of eye movement in the context of specific cognitive tasks. We are currently examining eye movements in HD patients and gene carriers during emotional and social informationprocessing tasks, analysing oculomotor parameters in relation to apathy and compared with healthy controls.

\section{ASSESSING LONGITUDINAL CHANGE IN IMAGING BIOMARKERS}

\author{
Elizabeth H. Aylward, Rachael I. Scahill, \\ Nicola Z. Hobbs, Sarah J. Tabrizi
}

\begin{abstract}
.
Introduction: Many studies support using structural MRI measures, particularly caudate volume, as outcome measures in HD clinical trials. Based on longitudinal imaging studies, different sample sizes have been estimated, and this may be due in part to different definitions of longitudinal change.

Objectives: The current study was designed to determine how effect sizes (1) are affected by differences in calculation of longitudinal change; (2) differ across the course of disease, based on different calcuation methods; and (3) differ based on the inclusion of various covariates.

Methods: Subjects were from TRACK-HD and included 56 preHD-A (>10.8 years from predicted diagnoses), 43 PreHD-B $(<10.8$ years from predicted diagnosis $), 51 \mathrm{HD} 1$ (early HD with $\mathrm{TFC}=11-13$ ), and $26 \mathrm{HD} 2$ (TFC $=7-10$ ). Controls $(\mathrm{N}=96)$ were age- and gender-matched. Data were
\end{abstract}


caudate volumes from baseline (Caudate1) and 24-month visits (Caudate3). Three methods were used to calculate longitudinal change: (a) Raw volume change: Caudate1 Caudate3; (b) Percent volume change: (Caudate1 - Caudate3)/ Caudate1; and (c) Corrected raw volume change: (Caudate1Caudate3)/Intracranial volume. For each HD group and each method of calculation, an effect size was derived showing the strength of the difference between the HD subgroup and controls.

Results: Regardless of the calculation used (raw, percent, corrected), the controls and PreHD-A group showed significantly less change than the other HD groups. For percent volume change, HD1 also showed more change than PreHD-B. Results were basically the same when ICV was used as a covariate, with or without including age and gender as covariates. For both corrected and uncorrected baseline volume, all HD groups showed significant correlations (or at least a trend) between baseline volume and percent volume change, but generally not between baseline volume and either raw or corrected volume change.

Conclusion: Effect sizes do not consistently change based on the method used for calculating longitudinal change. Percent difference (caudate volume change divided by baseline caudate volume) results in significant group differences in amount of longitudinal change, with higher percentages for groups further along in the course of the disease. When baseline caudate volume is not included in the formula, the rate of change stays relatively steady once atrophy begins (in the pre-HD group that is closer to onset).

\section{ASYMPTOMATIC ELDERLY GENE CARRIERS FOR HD}

\section{Sven Asger Sorensen}

Abstract. Recently we were informed about a 63 year old man who was clinically diagnosed with mild symptoms of Huntingtons disease, verified by molecular analysis. This man is registered in the Danish Huntington Register (DHR) that now contains more than 12.000 individuals with 1599 affected in 359 families. The man belongs through his father to a family in which we know of 43 verified cases of HD. The man had never heard about HD before and had no knowledge to the occurrence of this disease in his family. Neither the man nor his father are classified in the DHR as having HD. He told that his father who died at the age of 91 years did not have any symptoms of HD. It is not uncommon that persons with symptoms of HD claim to have no relatives with HD. In order to look for similar cases we have collected all registered individuals from the DHR of persons that have children with $\mathrm{HD}$, and more cases of the disease in their families, but have not been diagnosed themselves as having HD. A total of 37 persons - 20 females and 17 males - were encountered. Information from their relatives, death certificates, and where possible hospital records were negative about the occurrence of HD in these persons. Concerning the males, it is unlikely that their children with HD were illegitimate because the males were only selected for the study if they had relatives with HD. None of the 37 individuals were alive. The mean age at death was 68.9 years for females with a range of $26-$ 95 years, and 63.1 years for men with a range of $42-84$ years.
Two of the females, but none of the males, died at ages lower than 40 years which can be the reason why they did not have symptoms of HD. Eliminating these two persons, the mean age at death for females was 81.2 years, range 63 - 95 years. Huntington disease can have its onset at old age, particularly in persons with short CAG repeats in the low range and so, we can not disclose that the persons here reported would have presented symptoms if they have lived longer. We have no data on their CAG lengths, and it cannot be ruled out that the persons had alleles with short CAG repeats. But even then, our findings show that the occurrence of a HD allele not necessarily results in manifest disease. Thus it is likely that more individuals than hitherto suggested are gene carriers in the populations. Our results also shows the importance of a comprehensive family study when more cases of HD in the family are denied.

\section{AXONAL FIBRE CONNECTIVITY IN PREMANIFEST AND SYMPTOMATIC HUNTINGTON'S DISEASE: CROSS- SECTIONAL DATA FROM THE IMAGE-HD STUDY}

Govinda R Poudel, Juan F Domínguez D, Louisa Salmon, Andrew Churchyard, Phyllis Chua, Julie C Stout, Gary F Egan,

Nellie Georgiou-Karistianis

\section{Abstract.}

Introduction: The underlying mechanisms of cognitive deficits associated with Huntington's disease (HD), which are often present several years prior to clinical diagnosis; remain to a great extent unknown.

Objective: To investigate axonal fibre connectivity and the relationship between axonal microstructure and clinical, cognitive, and motor function in premanifest (pre-HD) and symptomatic (symp-HD) HD individuals. Method: Diffusion tensor imaging (DTI) data were acquired from 35 pre-HD, 36 symp-HD, and 35 controls as part of the Australian based IMAGE-HD study. Structural connectivity was mapped between 40 brain regions of interest using tractography. A network based statistical method was used to isolate brain networks disrupted in pre-HD and symp-HD, compared to controls.

Results: Pre-HD showed axonal disruption in a network connecting striatum with frontal and motor cortices. SympHD individuals showed more wide-spread disruption in cortico-cortical and cortico-striatal networks, compared with pre-HD. Radial diffusivity in the cortico-striatal tracts correlated with neurocognitive (i.e., SDMT \& STROOP) performances in both pre-HD and symp-HD.

Conclusion: We provide direct evidence of aberrant connectivity in structural networks related to neuropathology in $\mathrm{HD}$, thus suggesting that axonal integrity provides an early marker of clinical severity in HD. 


\section{BEHAVIOURAL AND PATHOLOGICAL PHENOTYPING OF THE C57BL/6J BACHD TRANSGENIC MOUSE MODEL OF HUNTINGTON'S DISEASE}

Susanna Mantovani, Rui Li, Richard Gordon, Vinod Kumar, Steven M. Taylor, Trent M. Woodruff

\begin{abstract}
.
Introduction: Huntington's disease (HD) is an incurable neurodegenerative condition characterised by progressive motor and cognitive dysfunction, and depletion of neurons predominantly in the striatum. Recently, a new transgenic mouse model of HD, called the BACHD mouse, has been generated, expressing the full-length human huntingtin gene (Gray et al., 2008). It is suggested to be particularly suitable for preclinical studies. Objectives: The majority of studies published using the BACHD model, have used this transgenic model on a FVB/N or a mixed genetic background. In this study, we carried out a series of behavioural and neuropathological tests on BACHD mice on a pure C57BL/6 J genetic background, since this is the most common mouse background used in preclinical research. Our work provides a solid starting point for the usage of these mice for drug testing, or for generating crosses with other transgenic models on the
\end{abstract} same background.

Methods: BACHD and age-matched WT control mice were examined at regular intervals up to 12 months of age. Mice were assessed for their motor and cognitive functions. We performed the CatWalk test for gait analysis; Grip strength analysis; Rotarod test to assess motor performance, Balance Beam Test to assess motor balance and coordination; Open Field test for locomotor and exploratory activity; Forced Swim test to measure depression levels and Elevated Plus Maze test to assesses anxiety levels. Brains from these mice were also analysed for signs of gliosis. Finally striatal volume size in BACHD and age-matched WT mice at 12 months of age were compared using anatomic $16.4 \mathrm{~T}$ MRI brain scans.

Results: C57BL/6J BACHD mice showed motor impairments on rotarod and balance beam starting from 3 months of age. At this age, no signs of depression were observed in the forced swim test. Open field tests showed the BACHD to be hypoactive compared to WT mice starting from 6 months of age. Interestingly, no alterations in the analysis of gait and grip strength were seen at the ages studied. The striatal volume was not different between BACHD and WT mice at 12 months of age as determined by MRI brain scans. Preliminary data also indicate apparent gliosis in the BACHD mice.

Conclusions: BACHD mice on the $\mathrm{C} 57 \mathrm{BL} / 6 \mathrm{~J}$ background show some features reminiscent of human HD, however they do not have clear changes in striatal volume up to 12 months. These results may be useful as a reference baseline in future experiments using C57BL/6J BACHD animals.

\section{BEING HUNTINGTONIAN: ABOUT ANOUCK}

\section{Valérie Pihet, Emilie Hermant}

Abstract. The aim of Dingdingdong - Institute for the Coproduction of Knowledge about HD is to create knowledge about HD, taking into consideration the expertise produced by sick persons, carriers at risk, families, as well as by physicians. Among other things, our Institute conceives portraits of persons affected by HD. These portraits are elaborated from different disciplines such as arts, social sciences and medicine. This method is an heuristically way to report the experience of the disease as a multidisciplinary issue. In this poster, we are presenting the portrait of Anouck, a woman in her late sixties affected by HD, who is still leaving in her apartment in Paris. Around Anouck, we show all the personal and technical attachments and links composing her care, as a map. Using actor-network theory and cartography methodology, this work reveals the multiple and heterogeneous links which composes the reality of her care.

\section{BIOMARKERS FOR HUNTINGTON'S DISEASE: A SYSTEMATIC REVIEW}

Tiago Mestre, Thilo Van Eimeren, Lina Winter, Loh Han Chern, Cristina Sampaio

\section{Abstract.}

Introduction: There is a need of new therapies for Huntington's disease (HD), namely, disease modifying treatments. A biomarker is an indicator of normal biological processes, pathogenic processes, or pharmacologic responses to a therapeutic intervention that can be objectively measured and evaluated. Resource to biomarkers in drug development can assist in capturing clinical relevant changes. The systematic assessment of biomarker studies in HD will help to identify the best candidates and assess the quality of biomarker development in HD.

Objective: Identify all studies conducted for biomarker development in HD and conduct a methodological appraisal of the identified studies.

Design: We conducted a systematic review of all studies conducted with the goal of biomarker development in participants with genetically confirmed HD. All longitudinal studies were included. Cross-sectional studies were included if a comparison between different stages of disease was made or a correlation with valid outcomes of severity within the same disease stage was considered. All types of biomarkers were included. Data abstracted from the studies included aspects of study design, methodology of data analysis, characteristics of participants and results.

Results: 282 studies were identified. 101 (35.8\%) studies were included. Of these, 76 were observational, including 38 (49.4\%) with a longitudinal study design. 61/92 (66.3\%) biomarkers studies were conducted with the goal of measuring disease progression, while 26/92 (28.3\%) studies were conducted as state biomarkers. Type of biomarkers studied: imaging $(n=48,47.5 \%)$, clinical $(n=27,26.7 \%)$, genetic $(n=11,10.9 \%)$, and biochemical $(n=13,12.9 \%)$. A minority of studies $(n=2,2.0 \%)$ selected a combination of biomarkers. Four studies included measures of reliability $(n=2)$ or accuracy $(n=2)$ of the candidate biomarker.

Conclusions: There are no established biomarkers for HD. The majority of the studies included in the current systematic review show methodological flaws in study design and data analysis. There is a need to implement reliability and accuracy 
measures for biomarker studies in HD. Standards for biomarker development in HD are warranted.

\section{BODY COMPOSITION OF PATIENTS WITH HUNTINGTON'S DISEASE. SPANISH STUDY OF THE EUROPEAN GROUP FOR HUNTINGTON'S DISEASE}

Jéssica Jannett Rivadeneyra Posadas, Esther Cubo Delgado, Cecilia Gil Polo, Natividad Mariscal Pérez, Diana Armesto Formoso, Ana Mateos Cachorro, Rafael J. A. Cámara, Asunción Martínez Descalls, Spanish European HD Registry

\begin{abstract}
.
Background: Bioelectrical Impedance Assessment (BIA) is a method which estimates body compartments. The imbalance between food intake and requirements, either by excess or deficit, has consequences for their development. Moreover, no data exists on body composition in patients with HD.

Objectives: To compare the body mass composition in terms of body mass index (BMI), body fat mass, fat free mass and total body water, and resting metabolic rate (RMR) between a representative sample of patients with HD and age-, gender-, and BMI-matched control subjects.

Methods: Cross-sectional case-control study of the European HD Registry. Patients with genetically confirmed HD and healthy matched controls were included. Body composition and BMI were measured using the single-frecuency (BIA) Tanita ${ }^{\circledR}$ BC418MA.

Results: Twenty four HD patients with $62.5 \%$ women and a mean age of $51 \pm 15$ years. According to the WHO classification of BMI, $4.2 \%$ were underweight, $70.8 \%$ were normal, and $25 \%$ were overweight. Twenty one controls were included and matched in terms of age and gender. Compared to controls, HD patients were underweight $(p=0.005)$, and with lower $\operatorname{RMR}(p=0.005)$, lower body fat mass $(p=0.039)$, fat free mass $(p=0.013)$, and total body water $(p=0.013)$. BMI was highly correlated with body fat mass ( $\mathrm{rs}=0.87$, $p<0.0001$ ), fat free mass ( $\mathrm{rs}=0.76, p<0.0001$ ), and RMR ( $\mathrm{rs}=0.81, p<0.0001)$ in controls, but in the HD group BMI was not correlated with fat free mass and RMR.

Conclusions: In this ongoing study, patients with HD had lower BMI, RMR, fat free mass, body fat mass, and total body water compared to controls. BMI as an estimator of nutritional state might be of limited use in HD because it overestimates the nutritional state. The BIA should be used to assess the nutritional state for further nutritional interventions because it provides a better estimation of the compartments of the body.
\end{abstract}

\section{BRAZILIAN VERSION OF THE SYMBOL DIGIT MODALITIES TEST (SDMT): PRELIMINARY ANALYSES}

Carina Tellaroli Spedo, Rodrigo Melo Conde, Gérson Silva Santos Neto, Isadora Chaves de Freitas, Millena Vaz da Costa Valadares, Mariângela Santos de Jesus, Leandro Ribeiro Azevedo, Silvana Batista Gaino, Amilton Antunes Barreira, Vitor Tumas, Vanessa Daccach Marques, Maria Paula Foss

\section{Abstract.}

Introduction: The SDMT is one of most commonly used tests in the standardized evaluations of patients with Multiple Sclerosis, Huntington Disease and Traumatic Brain Injury. This test usually access divided attention, visual scanning, tracking and motor and perceptual speed. In fact decrease information in processing speed is frequently cited as the earliest and most pervasive cognitive deficit associated with multiple sclerosis. In Brazil a formal standardization of administration of SDMT with the study of demographic data and comparisons between the applications forms (written or oral) were not showed previously.

Objectives: in the present study we aimed to provide standardization on procedures of administration to a Brazilian sample as provided in original SDMT, to investigate demographic influences and correlation among the forms of applications and finally, provide evidence of the validity of this Brazilian version to MS patients.

Method: the sample was constituted by young adults, with 20-59 years old from Southeast, Northeast and North region of Brazil. Fifteen patients with MS (mean 34.8 years old, 10 years of schooling and mean MMSE of 29.9 points) were compared with 56 healthy controls (mean 45.6 years old, 13 years of schooling and mean MMSE of 28.0 points). They completed the SDMT on both written and oral format. In the standard procedure of test the examinee make a training of the procedure followed by the written version and after, without training, the oral version of SDMT must be applied. The examinee has 90 seconds to complete the trial. The MMSE were also recorded.

Results: according to the demographic characteristics of the sample, only the schooling showed that better educated examinees have higher scores than less-educated examinees. The correlations between written and oral forms were 0.90 . There was a significant difference among $(p<0,001)$ in MS group (27.7 and 35.5) when compared with controls (41.0 and 46.0) with ANCOVA controlling for schooling.

Conclusions: schooling was the only demographic variable that interfered in SDMT performance. The written and oral administration demonstrated high correlation four Brazilian populations. There was some evidence of the validity of this version of the SDMT. Further studies about the reliability and validity with other clinical groups, including Hungtinton Disease, should be conducted to ensure the application of SDMT to Brazilian population.

\section{BURDEN OF HEALTHCARE RESOURCE UTILIZATION AMONG PATIENTS DIAGNOSED WITH HUNTINGTON'S DISEASE}

\section{ElizaBeth Grubb, Maureen Lage, Michael Treglia}

\footnotetext{
Abstract.

Objectives: The purpose of this retrospective, database analysis is to examine characteristics of patients diagnosed with Huntington's Disease (HD) in the United States and to compare healthcare resource utilization for patients pre and post initial diagnosis.

Methods: Data were obtained from the i3 Invision Datamart over the period January 1, 2006 through September 30, 2012. Study subjects had at least two (2) diagnoses of HD (ICD-9
} 
333.4) included in their administrative medical records, with the first date identified as the index date. All subjects were also required to have continuous insurance coverage from one (1)-year prior to the index date (pre-period) through 1-year after the index date (post-period.) Descriptive statistics were used to summarize the subjects' socio-demographic characteristics. The main results compared pre- and postperiod healthcare resource utilization. Paired $t$-tests were utilized for continuous variables and McNemer's test for categorical variables. The pre-specified level of statistical significance was $5 \%$.

Results: There were 94 subjects who met the study's criteria. The mean (SD) age was 49 (15) years, with females $(n=54)$ comprising $57.45 \%$ of the sample. The geographic distribution of the sample was: $12.77 \%$ Northeast, $34.04 \%$ Midwest, $31.91 \%$ South, and $21.28 \%$ West. All but one of the subjects were covered by a commercial insurance plan. Charlson Comorbidity Scores did not statistically significantly differ between the pre- and post-periods $(0.52$ [1.21] vs. 0.64 [1.11]; $p=0.3604)$. Compared to the pre-period, statistically significantly greater percentages of HD subjects had postperiod neurologist visits $(74.47 \%$ vs. $34.05 \%, p<0.0001)$ or were hospitalized $(19.15 \%$ vs. $8.51 \%, \quad p=0.0184$. Furthermore, post HD diagnosis, compared to pre HD diagnosis, patients had a significantly higher number of outpatient visits (19.69 [26.34] vs. 11.72 [21.95]; $p=0.0003)$, neurologist visits $(2.54$ [3.05] vs. 0.97 [1.70]; $p<0.0001)$, and hospitalizations $(0.28[0.66]$ vs. $0.11[0.40] ; p=0.0201)$.

Conclusions: In the 1-year period following receipt of a HD diagnosis, the burden of healthcare resource utilization statistically significantly increased compared to the year prior to the diagnosis. These results complement previously reported findings on the functional impairments associated with HD.

\section{CAN BEHAVIOURAL DECLINE IN HUNTINGTON DISEASE BE PREDICTED FROM NEUROPSYCHOLOGICAL MEASURES?}

Jillian McMillan, Robyn Langdon, Andrew Hayen, David Gunn, Elizabeth McCusker, Clement Loy, The Huntington Study Group COHORT Investigators

\section{Abstract.}

Introduction: Behavioural symptoms of Huntington disease (HD) are increasingly recognised for their significant impact on patients, their families and caregivers. Such symptoms are associated with frontal-striatal dysfunction, affecting an area of the brain also associated with executive functioning. Previous research suggests that performance on the Stroop test, a test of executive functioning, may be associated with these behavioural symptoms.

Objectives: Using clinical data from the COoperative Huntington Observational Research Trial (COHORT) we investigated whether the composite Stroop score, a specific measure of executive function, is associated with behavioural symptoms at baseline, after adjusting for clinical variables and general cognitive function (the Mini-Mental State Examination, MMSE). We performed the same analysis for prediction of behavioural decline 12 months post Stroop test. Methods: Data from 865 HD gene-positive participants with complete cognitive/behavioral data and a clinical diagnosis of HD (UHDRS motor rating of 4) was extracted from the COHORT database. We analysed the data using MannWhitney U tests, correlation analyses, linear regression and logistic regression. Regression analyses were adjusted for age, gender, education, cytosine-adenine-guanine (CAG) repeat length, and depression.

Results: No significant differences were found between participants with and without behavioural symptoms at baseline in terms of either Stroop or MMSE performance. For participants with behavioural symptoms at baseline, only the MMSE, CAG and depression were significantly associated with behavioural symptom severity. For participants without behavioural symptoms at baseline, depression and MMSE significantly predicted presence or absence of behavioural symptoms 12 months later.

Conclusions: The Stroop composite score was not associated with behavioural symptoms in HD, but the MMSE was. The impact of depression on neuropsychological functioning, limitations of the Stroop test, and the disunity of behavioural symptoms in HD are discussed in terms of specific neuroanatomical correlates within the fronto-striatal system. Further research into the relationship between executive functioning and behavioural symptoms in HD is warranted, particularly given the potential for neuropsychologists to detect early changes in cognitive and behavioural functioning, which could facilitate appropriately timed pharmacological and behavioural interventions.

\section{CAN WE PREDICT WHEN DRIVING IS NO LONGER SAFE FOR PEOPLE WHO HAVE HUNTINGTON'S DISEASE USING STANDARD NEUROPSYCHOLOGICAL MEASURES?}

Bonnie L. Hennig, Jessica Berenguer,

Richard F. Kaplan

\section{Abstract.}

Introduction: Early cognitive dysfunction in HD is typically of a subcortical frontal executive type, with bradyphrenia, poor spatial \& working memory, poor planning and organization, a lack of judgment, \& poor mental flexibility. Although there is a literature suggesting a correlation between deficits in speed of processing, working memory and executive function on driving competency, there is little direct evidence comparing these declines on tests to actual driving skills. The current case series examines the utility of specific neuropsychological measures in predicting actual driving competency.

Participants and Methods: Fifty-six patients at the UConn HD Program underwent yearly neuropsychological evaluations. From a battery of 12 tests, four scales were chosen a priori to predict driving impairment. These evaluations included the RBANS Coding Test, Trail Making Test Part B, Stroop Color-Word \& California Computerized Assessment Package (CalCAP). Patients were pre-screened on the basis of scores on the Total Function Capacity (TFC) test. Those scoring below a 7 were judged to be clinically impaired \& required no further neuropsychiatric testing. Those with higher scores, whether asymptomatic, mildly or moderately symptomatic, were tested as above. 
Results: Out of the total of 56.27 patients scored in the normal range \& no further action was taken. The remaining 29 patients scored below threshold suggestive of neuropsychological impairment. These 29 patients with documented HD were found to have results on the evaluation battery within the impaired range (1.5. SD below age corrected normative data). A referral to the Connecticut Department of Motor Vehicles (DMV) for a driving evaluation was subsequently made for these 29 patients. Twenty-one of the 29 patients had either their driving license suspended or voluntarily stopped driving. Five patients referred to DMV are pending a driving evaluation \& the remaining 3 patients passed their driving evaluation. The 3 patients who passed the driving evaluation have been required to return to the DMV every 6 months for ongoing driving test surveillance.

Conclusion: In this non-random case series, there is a strong relationship between scores on a battery of neuropsychiatric testing \& driving competency. The relationship in this preliminary data may provide information to guide a more formal assessment of measures to reliably determine driving competence \& the decision to refer to the DMV.

\section{CARDIAC SAFETY OF PRIDOPIDINE, A TREATMENT FOR HUNTINGTON'S DISEASE, AS EVALUATED IN HV AND PATIENTS}

Borje Darpo, Esther Lukasiewicz-Hagai,

Ofer Spiegelstein, Anna Wickenberg, Merav Bassan, Laura Rabinovich-Guilatt

\footnotetext{
Abstract.

Background: Pridopidine is currently under clinical development by Teva Pharmaceuticals for the treatment of motor symptoms in patients with Huntington's disease. In a multiple-dose study (Study ACR16C018), higher doses of pridopidine than previously investigated were administered to healthy volunteers, and extensive ECG assessments were conducted. A significant QTcF prolongation was observed to a level that has not previously been noted.

Objectives: (1) To evaluate the concentration-response of the QTc effect of pridopidine (2) To overview the effect of pridopidine on QTc prolongation based on previous clinical studies. (3) To evaluate adverse events, which may be related to delayed cardiac repolarization in patients with $\mathrm{HD}$.

Methods: The relationship between QTcF changes and pridopidine plasma concentrations in study ACR16C018 was quantified using a linear mixed-effects modeling approach. Cardiac safety data from 3 studies in HD patients including 490 patients receiving pridopidine in doses ranging from 20 $\mathrm{mg}$ daily (10 mg BID) to $90 \mathrm{mg}$ daily (45 $\mathrm{mg}$ BID) were reviewed.

Results: The PK-QTcF effect analysis indicated a concentration-dependent effect on the QTc interval, which could be of clinical significance. Pridopidine has been studied in 3 randomized, placebo-controlled trials in patients with HD with almost 500 patients on active treatment (up to $45 \mathrm{mg}$ BID). Notably, in these trials there were no patients, at any time point, with a QTcF value exceeding $500 \mathrm{~ms}$. Only one patient was observed with $\mathrm{QTcF}>480 \mathrm{~ms}$; one patient demonstrated $\Delta \mathrm{QTcF}>60 \mathrm{~ms}$ and the proportion of patients with $\Delta \mathrm{QTcF}>30 \mathrm{~ms}$ was either comparable across treatment
}

groups (MermaiHD study) or higher in the placebo group (HART study). Overall, the incidence of cardiac adverse events was low and data do not suggest that pridopidine confers a proarrhythmic risk. There were no events of cardiac arrest, sudden death or ventricular arrhythmias.

Conclusions: The QTc effect should be further characterized in the targeted patient population. Based on the described effect by pridopidine on QTc interval, we recommend criteria for ECG monitoring in patients. Available clinical data suggest that pridopidine can be safely administered to patients with HD providing that adequate safety measures are implemented, and further studies are planned to demonstrate a favorable benefit risk profile.

\section{CEREBROSPINAL FLUID AS A SOURCE OF BIOMARKERS FOR NEURODEGENERATION IN HUNTINGTON'S DISEASE: COMPARISON OF ELEVATED PROTEIN TAU AND NEUROFILAMENT H}

Sigurd D. Süssmuth, Helen Belz, Vera Lehmensiek, Patrick Weydt, G. Bernhard Landwehrmeyer

\section{Abstract:}

Introduction: Cerebrospinal fluid (CSF) biomarkers are important diagnostic tools in the work-up of patients with cognitive impairment, but there is still very limited knowledge about CSF biomarkers in HD. Objective: Comparison of neurdegenerative CSF biomarkers protein tau and neurofilament protein $\mathrm{H}(\mathrm{NfH})$ in relation to clinical parameters and different disease stages of $\mathrm{HD}$.

Methods: CSF samples of 30 well characterized HD gene mutation carriers from the REGISTRY cohort (age: 26-70 y; premanifest: $n=6$; stage I+II: $n=17$; stage III+IV: $n=7$ ) and of 33 age-matched controls suffering from Bell's palsy (idiopathic facial nerve paralysis) were analysed. Parameters of basic CSF analysis (cell count, lactate, total protein, concentrations of albumin and immunoglobulins as well as oligoclonal IgG bands) were normal. Commercially available ELISAs were used for the measurements of CSF tau and NfH. Results: There was a significant but only moderate increase in CSF tau concentrations of HD patients compared to the controls (mean $224.7 \mathrm{ng} / \mathrm{L}$ [80.0-424.1] vs. $183.4 \mathrm{ng} / \mathrm{L}$ [85.2313.5], $p=0.023)$. CSF concentrations of $\mathrm{NfH}$ in the HD cohort were almost twice the NfH levels of the controls (342.7 $\mathrm{ng} / \mathrm{mL}$ [136.4-688.7] vs. $187.6 \mathrm{ng} / \mathrm{mL}$ [26-352], $p<0.001)$. There was a correlation of both markers with age, but not with disease duration, the UHDRS total motor score or the sum score of a cognitive assessment battery. Both the values of $\mathrm{NfH}$ and tau did not correlate with CSF total protein content. Conclusion: Elevated CSF tau as well as NfH levels indicate neuroaxonal damage in $\mathrm{HD}$, but $\mathrm{CSF} \mathrm{NfH}$ was superior to tau in this respect. Longitudinal CSF data are needed to evaluate the utility of these proteins as state markers. 


\section{CHANGES IN INTERNEURON POPULATIONS IN THE CEREBRAL CORTEX IN HUNTINGTON'S DISEASE}

\author{
Nasim Mehrabi, Henry Waldvogel, Lynette Tippett,
} Virginia Hogg, Richard Faull

Abstract.
Introduction: Huntington's disease (HD) is a Introduction: Huntington's disease (HD) is a
neurodegenerative disorder with variable symptoms including chorea, cognitive, mood and neuropsychological changes. According to our recent studies, variable symptomatology of $\mathrm{HD}$ is associated with variable pyramidal cell loss in the cortex. GABAergic interneurons are inhibitory neurons that modulate the activity of pyramidal neurons in the cortex, thereby determining cortical output. To further extend our previous results, we are investigating the correlation between the pattern of GABAergic interneuron loss in the cerebral cortex and the phenotypic variability in HD.

Objectives: To investigate changes in interneuron populations in the primary sensory cortex and the superior parietal cortex, and to determine whether interneuron loss also correlates with symptom profile. Methods: A double blinded study was carried out using unbiased stereological cell counting to quantify two major types of GABAergic interneurons in the primary sensory cortex and superior parietal cortex of $10 \mathrm{HD}$ and 12 neurologically normal post-mortem human brains. Two different calcium binding proteins, calbindin-D28k (Calb) and parvalbumin (Parv), were used to label interneurons. The HD cases are categorized into three dominant symptom groups ("mood", "motor" and "mixed") based on detailed data of their symptomatology.

Results: According to our preliminary data, there is a heterogeneous loss of $\mathrm{Calb}+$ interneurons in the sensory cortex of the HD cases compared to control cases. We have observed a significant loss of Calb+ interneurons (67\% loss) in HD cases with major "motor" disorder, but not in cases with mainly "mood" or "mixed" symptoms in the primary sensory cortex. Parv+ interneurons were preserved in all HD cases. In superior parietal cortex, however, no significant changes were observed in the total number of $\mathrm{Calb}+$ and Parv+ interneurons in HD cases compared to normal Similarly, with reference to symptomatology (mood, motor and mixed), both Calb + and Parv + interneurons are spared in the superior parietal cortex.

Conclusion: These results suggest an important association between the variable pattern of interneuronal loss in the cerebral cortex and the variable symptomatology in HD. Also, these findings show a differential pattern of interneuron loss in the primary functional area of the parietal lobe (the primary sensory cortex) compared to an association area of the parietal lobe (the superior parietal cortex).

\section{CHARACTERISATION OF A LARGE GROUP OF INDIVIDUALS WITH HUNTINGTON'S DISEASE AND THEIR RELATIVES ENROLLED IN THE COHORT STUDY - A LONGITUDINAL ANALYSIS OF DATA}

Anita Goh, Samantha Loi, Stephanie Antonopoulos, Edmond Chiu, HSG COHORT investigators and coordinators

\section{Abstract.}

Annual data was collected from HD families in the USA, Canada and Australia from 2006 - 2011. Analysis was undertaken on all randomised participants $(n=2262)$ over four time points (baseline to year 3 ) in four groups of participants - premanifest/manifest HD [ $n=1301]$, adults/ older adolescentsat-risk $[n=423]$, adults at secondary risk $[n=12]$, controls $[n=501]$, and gene negative adults $[n=25]$. Results: Univariate general linear model procedures analysed longitudinal data, using Greenhouse-Geisser corrections where appropriate. There were statistically significant main effects for MMSE, TFC, Independence, and Motor scores for the Manifest/pre-HD group, indicating that these participants progressively worsened on these scales over the four time points. For all measures, pairwise comparisons revealed significant deterioration between each time point. MMSE. $\mathrm{F}=37.72$, $p=0.000$, partial eta squared $=0.09$. TFC. $\mathrm{F}=98.57, p=0.000$, partial eta squared $=0.21$ INDEP. $\mathrm{F}=121.91, p=0.000$, partial eta squared $=0.25$ MOTOR. $\mathrm{F}=87.22, p=0.000$, partial eta squared $=0.19$. There was insufficient sample size to analyse the adults-at-secondary-risk group. For all other groups, there was no statistically significant change in MMSE, Independence, and most TFC and Motor scores over all time points. Exceptions: Adult/ Older adolescent-at-risk group showed a significant decrease in TFC score from Baseline to Year 1 $(p=0.04)$ but no other significant changes at other time points; and the Adult/ Older adolescent-at-risk group revealed a change in motor score over time $(\mathrm{F}=6.96, p=0.001$, partial eta squared $=0.08)$ - post-hoc analyses revealed significant change between baseline and Year 3 and between Year 1 and Year 3.

Conclusions: Participants with manifest or premanifest HD are deteriorating in motor function, cognitive function, level of independence, and total functional capacity over time, whereas their family members are not. There was no deterioration in behavioural scores over time in any group. There were significant limitations on the scores on clinical measures for all groups except for the Premanifest/ manifest HD group, indicating a lack of sensitivity in these scales for non-clinical populations - who all scored highly (no impairment) on all tasks. All values were heavily skewed. There were floor effects on the UHDRS Motor and Behavioural scale, and ceiling effects on the UHDRS Independence, TFC, and MMSE scales. More sensitive measures are needed in non-clinical groups. 


\section{CLINICAL PROFILE OF HUNTINGTON'S DISEASE PATIENTS FROM TWO TERTIARY CENTERS IN RIO DE JANEIRO, BRAZIL}

\author{
Mariana Spitz, José Marcelo Ferreira Bezerra, João \\ Santos Pereira
}

\begin{abstract}
.
Introduction: Huntington's disease is a genetic neurodegenerative condition characterized by chorea, dementia and psychiatric abnormalities. The clinical diagnosis can be supported by brain MRI and confirmed by genetic analysis.

Methods: Retrospective analysis of files from 27 patients from two tertiary health centers in Rio: Hospital dos Servidores do Estado e Hospital Universitário Pedro Ernesto. Of these, 12 patients were excluded because of unavailability of the genetic test. The remaining 15 were divided in three groups according to the number of CAG repetitions on chromosome 4: Group I, 37-39; Group II, 40-41 and Group III, 44-50. The parameters analyzed were: age of disease onset, initial symptoms, present symptoms, disease duration, familial history and correlation with number of repetitions.
\end{abstract}

Results: In Group I (5 patients), the average age of disease onset was 50,6 years, with an average disease duration of 7 years. In Group II (6 patients), the average age of onset was 50 years, with an average disease duration of 12,3 years. In Group III (4 patients), the average age of onset was 35, 7 years, with an average disease duration of 9,2 years. The rate male:female in Groups I, II and III was, respectively, 1:4, 6:0 and $2: 2$. A positive family history was observed in $80 \%$ of the total amount of patients - those with a negative family history belonged to Group II. In all the groups the predominant initial symptom was chorea, which was present in all the patients at some point of the disease. Neuroimaging studies were available to a minority of patients, so they were not evaluated in the present study. Main current symptoms were: in group I, sleep disorders, irritability and dysphagia; in group II, dysphagia, followed by depression, dementia and irritability and in group III, irritability, dysarthria and chorea.

Conclusion: A higher number of CAG repetitions was associated with an earlier age of disease onset, but this difference was very little comparing $37-39$ and $40-41$ repetitions. There was a male predominance $(1.5: 1)$ in the sample evaluated. The main symptom throughout the course of the disease was chorea. A positive family history was not present in $20 \%$ of the patients, reaffirming the need of a diagnostic suspicion when dealing with a typical clinical picture. In spite of the advances of diagnostic tools for HD, we still face difficulties in obtaining genetic test and brain MRI in our public practice in Brazil.

\section{COGNITION IN HUNTINGTON'S DISEASE IN MANIFEST, PREMANIFEST AND CONVERTING GENE CARRIERS OVER TEN YEARS}

Ellen Hart, Eve Dumas, Erik Giltay, Huub Middelkoop, Raymund Roos

\section{Abstract.}

Background: Cognitive decline in Huntington's disease (HD) remains an area of inconsistencies, especially far from disease onset.
Objective: To clarify the course of cognition in premanifest HD.

Methods: Twenty-six premanifest HD, 19 manifest HD, and 87 control subjects were followed for ten years, using an extensive cognitive battery. Differences in baseline levels and change over time, on four factors (motor speed, global cognition, executive functioning $(\mathrm{EF})$, and memory) were examined, using multilevel regression analyses. Converters were additionally analysed as a separate group. Also, the influence of motor speed and predicted years to disease onset on the cognitive factors was studied.

Results: Manifest HD subjects showed lower baseline scores compared to controls on the motor speed $(p=0.002)$, memory $(p<0.001)$ and EF $(p<0.001)$. They additionally deteriorated over the ten-year follow-up on memory $(p=0.01)$. Converters deteriorated on $\mathrm{EF}(p=0.04)$. Further analyses of premanifest subjects 'far from and close to predicted onset' revealed lower baseline scores for the 'close' group on $\mathrm{EF}$, as compared to controls $(p=0.001)$. They also deteriorated on memory $(p=0.01)$. Motor speed substantially mediated the results of the three cognitive factors; when added as covariate to the model several baseline and slope differences for the cognitive factors ceased to be significant.

Conclusions: Memory and EF are highly sensitive for ascertaining deterioration in premanifest HD gene carriers, especially in subjects close to onset. Lack of deterioration for the subjects further away from onset suggests that both domains are largely unaffected in those far from onset. Also, motor influence on cognition is substantial and should be taken into account in cognitive HD research.

\section{COGNITIVE FLEXIBILITY DURING A SET RESPONSE SHIFT TASK IN HUNTINGTON'S DISEASE: 30 MONTH DATA FROM THE IMAGE-HD STUDY}

Juan F Domínguez D, Julie C Stout, Marcus A Gray, Louisa Salmon, Andrew Churchyard, Phyllis Chua, Beth Borowsky, Gary F Egan, Nellie GeorgiouKaristianis

\section{Abstract.}

Introduction: Cognitive flexibility, the executive capacity to adaptively shift attention among stimuli or responses according to context, is impaired in Huntington's disease (HD). Task-switching paradigms assessing cognitive flexibility have previously been shown to activate corticostriatal networks and to deactivate areas of the default-mode network, including medial prefrontal cortex and posterior cingulate cortex/precuneus.

Objectives: We aimed to investigate the progression over 30 months of neural responses evoked by a set response shift (SRS) task in cortico-striatal and default-mode networks in premanifest (pre-HD) and symptomatic (symp-HD), as well as healthy controls.

Methods: Twenty-six pre-HD, 11 symp-HD and 23 agematched (to pre-HD) healthy control participants underwent fMRI scanning at three time points (baseline, 18 months and 30 months) as part of the Australian based IMAGE-HD study. Participant inclusion was based on their ability to perform at $>70 \%$ on the SRS (alternate condition). Voxel-wise analyses 
modelled linear change across time within each group and longitudinal differences between the groups.

Results: Despite similar performance accuracies across groups, medial prefrontal cortex and posterior cingulate cortex/precuneus became progressively more deactivated across time in symp-HD participants during task switching, while no change was evident in pre-HD and control participants.

Conclusions: The progressive change in functional brain response in symp-HD participants represents the first evidence of a longitudinal compensatory response in the default-mode network. These results shed light on how compensatory mechanisms may unfold throughout the course of the neurodegenerative process, and provide evidence that change in default-mode network function may be a sensitive target region for future therapeutic intervention studies.

\section{CORRELATION OF CAG REPEAT LENGTH BETWEEN THE MATERNAL AND PATERNAL ALLELE OF THE HUNTINGTIN GENE: EVIDENCE FOR ASSORTATIVE MATING}

Peggy Nopoulos, Michael McHugh, Jessica Lee, Eric Epping

\footnotetext{
Abstract.

Background: While Huntington's Disease (HD) is known to be caused by a CAG triplet repeat in the gene Huntingtin (HTT), the effect of CAG repeats on brain function below disease threshold has not been studied thoroughly. Although HTT has not directly been associated with variance in behavior, it is critical for brain development and therefore may associated with variance in brain structure and function. We sought to evaluate the possibility of assortative mating in a group of subjects recruited from the community and with no family history of Huntington's.

Methods: As a control group for a study on children at risk for HD, healthy children ages 6-18 years of age were recruited from the surrounding community of our hospital via advertising. Several children who participated were siblings and to avoid the confound of genetic relatedness, in the cases in which there were 1 or more siblings, the participants were randomly deleted and only 1 child from each family was included. The total sample consisted of 85 children. The first 47 consecutive subjects were collected from 2009-2010 and a second set of subjects, $n=38$ were recruited in 2011-2012. These were treated as mutually exclusive, independent samples, in order to have higher confidence in the findings. Each child provided either blood or saliva for genetic analysis For research purposes, CAG repeats are quantified in the Huntingtin gene (HTT). The longest allele is designated as Allele1 and the shorter allele is Allele2. A simple correlation was run, within subjects, between Allele1 and Allele2. As the allele distribution is not normally distributed, a non-parametric Spearman's correlation was used. Results: The first sample of 47 children showed a significant correlation of $r=0.511$, $p=0.0002$. This observation confirms that longer Allele1 lengths are positively associated with longer Allele2 lengths. The correlation for the 2nd, independent sample, was similar at $r=0.528, p=0.0007$. Conclusion: This simple analysis of a unique data set shows evidence that there is assortative mating
}

in regard to CAG length of HTT. That is, the length of CAG repeat in the maternal allele of HTT strongly correlates with the length of the CAG repeat of the paternal allele of HTT, suggesting that the male and female pair mated based on the common feature of having a similar genotype (length of CAG repeat).

\section{CORTICAL THICKNESS AND BASAL GANGLIA VOLUME ALTERATIONS IN HUNTINGTON'S DISEASE: RELATION TO OCULOMOTOR FUNCTION}

Gina Caetano, Filipa Júlio, Gil Cunha,

Cristina Januário

\section{Abstract.}

Inroduction: This study aims at a three-years follow-up of Huntington's Disease (HD) participants, matched for healthy ageing. Here we present the first results from Year 1 on morphometry and its relation to oculomotor function.

Methods: These results correspond to a sample of twentyeight participants: 12 controls, 8 premanifest HD (preHD), and 7 early-HD (Stage1). Participants underwent two structural MRI scans (3T Siemens scanner) and data were processed with FreeSurfer5.1. Estimates of volumes of interest were extracted (bilateral Caudate and Putamen) and normalized to account for estimated total intracranial volume. Surface-based cortical thickness analysis was performed fitting a general linear model at each surface vertex (clusterwise correction for multiple comparisons). Oculomotor function was studied through horizontal saccadic tasks: prosaccades (PS), antisaccades (AS), 1-or-2 back memory prosaccade (MPS), and 1-or-2 back memory antisaccade (MAS). Correlation of saccadic measures (percentage of express errors and direction errors) with morphometric data was performed.

Results: Early-HD participants have significantly smaller putamen and caudate volumes than the preHD/control groups. Cortical thinning in early-HD is observed bilaterally throughout an extensive region of the cortex, compound primarily of superior frontal/parietal/temporal, inferior parietal and lateral occipital regions. In comparison with the preHD group, thinning is mostly observed on the left hemisphere parietal and temporal regions. Finally, differences between preHD and controls were not identified at the cortical level. In preHD, direction errors (AS) correlated significantly with striatal volumes $(p<0.05, r<-0.65)$. In early-HD, express errors (PS,AS, and MPS/MAS) correlated significantly with striatal volumes $(p<0.05, r<-0.8)$.

Remarks: These results enable to pinpoint neurodegeneration at different stages of HD, in agreement with known disease neuropathology and its relation to oculomotor function. Moreover, the hypotheses that cortical neuronal death in HD spreads from sensory areas (visual, motor) to parietal, frontal and temporal regions may best be in line with our findings. 


\section{CURRENTLY PREVALENCE OF HUNTINGTON'S DISEASE IN FEIRA GRANDE-ALAGOAS/NORTHEASTERN BRAZIL}

\author{
Maria Aparecida Santos de Souza Alencar
}

\section{Abstract.}

Background and Aims: Huntington's disease (HD) is a congenital neurodegenerative disorder that affects a lot of people around the world. In Maracaibo (Venezuela), the incidence is high, and although there is not an official statistic of the cases in Brazil, according to the HD Brazilian Association $(\mathrm{ABH})$, ca. 1,000 families suffers with this disease. However, after clinical and molecular analysis of patients with symptoms in Feira Grande, a small city (about 22,000 inhabitants) of the State of Alagoas (Brazil), 22 patients were identified with HD in 2005. It means that the prevalence was one for each 1,000 births. Some couples affected for HD have an expressive number of children and there were not public politics to work this question, especially genetic counseling. The purpose of the present work was to analyze the prevalence of the population currently affected by HD in this city.

Methods: All the families with HD reported cases were interviewed with the use of questionnaires and the diagnosis referred. The familiar heredrograms of the families, constructed in September 2005, were used to analyze the changes in the number of patients with $\mathrm{HD}$, especially if new cases arose. Results As from the dada, some changes could be observed: 07 patients died and a new case arose, totalizing 16 patients. So that, in Feira Grande city there is one affected person per each 1,375 inhabitant.

Conclusion: The prevalence of HD in Feira Grande, where there are a lot of couples affected for HD, is one of the highest in Brazil.

\section{DAILY CARE REHABILITATION CENTER WITH A COMMUNITY APPROACH FOR PEOPLE WITH HUNTINGTON'S DISEASE.}

Valeria Rey, Paulina Lorca, Daniela Alburquerque, Olga Benavides, Pedro Chana-Cuevas

Abstract. This program aims to maintain and improve levels of social and occupational participation commensurate with their abilities and interests

Method: In an open study is controlled 7 patient with Huntington disease 8.6 years of evolution, posing a severe compromise and require assistance, according to the Clinical Global Impression scale and with a important functional compromise of 20 according to the UHDRS, on the independence scale. They applied the Volitional Questionnaire and Huntington Quality of Life scale. The users (patients) participate daily on Clubhouse model, which are scheduled routine daily activities, workshop and other areas associated with the interests of users, focusing on therapeutic and functional objectives, set individually and participatory. Results are expressed as mean and range.

Results: Evaluating the quality of life in item (cognition and physical condition) 108.7 (73 to 138) at the end 89.1 (68 to
124), the emotional condition baseline 79.0 (48 to 97) at the end of 70,3 (53 to 63), 16.0 Services ( 4 to 24 ) and at the end 7.1 (4 to 10 ). The questionnaire basal volitional 31.2 (14 to 48 ) and at the end 41.2 (22 to 55).

Conclusion: The rehabilitation process impacts improving the quality of life and improved volitional aspects especially in patients with Huntington's disease in advanced stage.

\section{DESIGN OF THE DOSE-RANGE FINDING (DRF), RANDOMIZED, DOUBLE-BLIND, PLACEBO-CONTROLLED STUDY, EVALUATING THE SAFETY AND EFFICACY OF PRIDOPIDINE FOR SYMPTOMATIC TREATMENT IN PATIENTS WITH HUNTINGTON'S DISEASE}

Bernhard Landwehrmeyer, Ralf Reilmann, Karl Kieburtz, Esther Lukasiewicz-Hagai, Eli Eyal, Anna Wickenberg, Merav Bassan

\section{Abstract.}

Introduction: Huntington's disease (HD) is a neurodegenerative disorder with an autosomal dominant mode of inheritance, associated with a triad of motor, behavioral, and cognitive symptoms. A number of medications are prescribed to ameliorate the motor and emotional problems associated with HD however; the scientific evidence for the usefulness of various drugs is poor. Pridopidine belongs to a new class of pharmaceutical agents, the dopidines, which have dopaminergic stabilizing properties. Dopaminergic stabilizers can both enhance and counteract dopamine dependent functions in the central nervous system (CNS), depending on the initial level of dopaminergic activity. As was previously indicated in two clinical studies in HD patients, pridopidine could have an effect on voluntary motor impairment, with potentially increased effect in higher doses. Objectives: The primary objective of this study is to assess the efficacy and dose response of pridopidine 45 to $112.5 \mathrm{mg}$ twice daily (bid) on motor impairment in patients with HD after 12 weeks of treatment using the Unified Huntington's Disease Rating Scale (UHDRS) Total Motor Score (TMS). Methods: This is a multicenter, multinational, randomized, parallel-group, double-blind, placebo-controlled study to compare the efficacy and safety of pridopidine in the treatment of motor impairment in HD. It is planned to enroll a total of 250 patients (50 patients within each treatment arm). Patients will be equally randomized $(1: 1: 1: 1: 1)$ to receive pridopidine $45,67.5,90$, or $112.5 \mathrm{mg}$ or placebo bid for 12 weeks, including a 4 -week progressive titration period. The primary endpoint for this study is change from baseline in the UHDRS-TMS at week 12. Additional scales to evaluate global and functional impact of pridopidine treatment are being analyzed as secondary endpoints (including CIBIC-Plus, PDS, and the UHDRS functional scales). Complementary motor assessments will also be done, including PPT, TUG, and Q-motor. Safety and PK of the treatment will be evaluated, including extensive centralized ECG assessments throughout the study period.

Conclusion: There is a significant unmet medical need to ameliorate symptoms of HD. This study will provide essential data towards possible effective and safe use of pridopidine for HD patients. Enrollment for this study will start in Q3 2013. 


\section{DESIGN-BASED STEREOLOGICAL ASSESSMENT OF THE HEART ATROPHY CAUSED BY HUNTINGTON'S DISEASE IN TRANSGENIC MICE: PRELIMINARY RESULTS}

Fernando Vagner Lobo Ladd, Amanda Lopes Moreira, Aliny Antunes Barbosa Lobo Ladd, Antonio Augusto Coppi

\section{Abstract.}

Huntington's disease (HD) is an autosomal dominant hereditary and fatal neurodegenerative disorder caused by mutation in the HD gene, characterized by the progressive degeneration of the striatum and the corticostriatal pathway. However, recently, in addition to changes in the central nervous system have also been described changes in peripheral organs, especially and potentially dangerous, disorders in cardiovascular system, with autonomic imbalance and structural alterations. Epidemiological studies have shown that cardiac insufficiency is an important cause of death among HD-patients (30\%) and alterations in the autonomic nervous system (ANS) have been described, which are characterized by a decrease in cardiac modulation, increasing the risk of cardiac syncope. Since 1996 when the transgenic mouse strains where developed, several transgenic strains are widely used to study the effects of mutant huntingtin ( $\mathrm{mhtt}$ ) in several tissues, and this models are suitable for studying some aspects of the Huntington's disease. In this study, was used left ventricles from 12-week old male transgenic R6/2 mice (G2) $(n=5)$ and the respective wildtype littermates (G1) $(n=5)$. The myocardium and lumen of the left heart ventricle volume was estimated by Cavalieri's principle and the total volume of the left ventricle is a sum of both. This method is the most accurate and trustworthy stereological procedure for cavity organs. Preliminary results are shown as mean (Coefficient of variation): The myocardium volume of left heart ventricle was: $\mathrm{G} 1=64.1 \mathrm{~mm}^{3}(0.20)$ and $\mathrm{G} 2=48.04 \mathrm{~mm}^{3}$ (0.20); The lumen volume was: $\mathrm{G} 1=4.96 \mathrm{~mm}^{3}(0.57)$ and $\mathrm{G} 2=2.45 \mathrm{~mm} 3(0.44)$; The total left ventricle volume was: $\mathrm{G} 1: 69 \mathrm{~mm}^{3}(0.20)$ and $\mathrm{G} 2=50.49 \mathrm{~mm}^{3}(0.18)$. The volumetric differences between mean groups, were significant for myocardium $(p=0.046)$ and total volume of left ventricle $(p=0.032)$ and were not significant for lumen $(p=0.058)$, here we can observe a border line effect. So, the volume of myocardium and volume of left ventricle in transgenic mice decrease $25 \%$ and $26 \%$ respectively. Therefore, this preliminary results provides morphoquantitative data of the cardiac structure in this mouse model of Huntington's disease, showing a marked atrophy in mice heart, what could to imply in function disorders, which will studied in the next step of this study.

\section{DESTINATION MEMORY IN PATIENTS WITH HUNTINGTON'S DISEASE: "WHERE DID I PUT THE KEYS?"}

Philippe Allain, Christophe Verny, Marie Caillaud, AdrIana Prundean, Clarisse Scherer, Mohamad el Haj

\begin{abstract}
.
Introduction: Destination memory, a component of episodic memory, refers to the ability to remember the destination of produced events (e.g., "Where did I put the keys?"). This memory component is found to be deteriorated in several neuropsychological populations.
\end{abstract}

Objectives: Episodic memory has been found to be impaired in patients with Huntington's disease (HD), but destination memory has never been tested in these patients. In this study, we investigated destination recall in a group of HD patients recruited from the Huntington's patient population receiving annual medical and neuropsychological monitoring in the Department of Neurology of the University Hospital of Angers.

Methods: Fourteen French speaking HD patients ( 7 women and 7 men; mean age $=47.71, \mathrm{SD}=9.57$; mean years of schooling $=12.41, \mathrm{SD}=1.41$ ) with clinically diagnosed and genetically confirmed Huntington's disease [mean CAGlength $=44.6, \mathrm{SD}=2.8)]$ and 16 age, gender, and education matched healthy control subjects ( 8 women and 8 men, mean age $=49.32, \mathrm{SD}=12.44$; mean years of schooling $=13.72$, $\mathrm{SD}=2.87$ ) voluntarily took part in the study. HD patients were early in the course of the disease (mean duration of symptoms $=5.4, \mathrm{SD}=3.2$ years; mean score on the Unified Huntington Disease Rating Scale motor scale $=23.62$, $\mathrm{SD}=17.87$; mean Mattis Dementia Rating Scale score $=136.5$, $\mathrm{SD}=5.2$ ). In order to assess their destination recall, participants were asked to put 6 familiar objects (e.g. eyeglasses) in a $20 \times 20 \mathrm{~cm}$ black square box and 6 other familiar objects in a $20 \times 20$ white square box. On a 15 -min delayed recognition task, participants were exposed to the 12 objects and were required to remember the destination to which each object was originally designated (i.e., the black or the white square box). Results Huntington's Disease patients showed poorer $(p=0.01)$ destination recall (mean score $=8.71$, $\mathrm{SD}=0.71$ ) than control participants (mean score $=10.63$, $\mathrm{SD}=0.49$ ). The destination memory deterioration as seen in Huntington's Disease patients was found to be significantly correlated $(\mathrm{Rho}=0.57, \quad p<0.05)$ with their episodic performance, as assessed with the Hopkins Verbal Learning Test.

Conclusion: Our results confirm that destination memory could be early impaired in HD patients. These findings highlight new tool for assessing episodic memory impairments in patients with HD.

\section{DIETARY INTAKE OF PATIENTS WITH HUNTINGTON'S DISEASE. SPANISH MULTICENTER STUDY OF THE EUROPEAN GROUP FOR HUNTINGTON'S DISEASE}

Jéssica Jannett Rivadeneyra Posadas, Esther Cubo Delgado, Cecilia Gil Polo, Natividad Mariscal Pérez, Diana Armesto Formoso, Ana Mateos Cachorro, Rafael J. A. Cámara, Asunción Martínez Descalls, Spanish European HD Registry

\section{Abstract.}

Background: The energy and nutrient needs of a healthy person are determined by the basal metabolism, the physical 
activity level, and the diet-induced thermogenesis. The dietary intake in HD is not well known at the moment.

Objectives: To describe the dietary intake in patients with HD.

Methods: Spanish multicenter, cross-sectional study of the European Register of HD. Dietary intake was assessed by a 24-hours recall questionnaire. Macro and micronutrients and energy intake information were obtained using the software Alimentación and Salud, version 2.0.

Results: 128 patients were included, of which $53.9 \%$ were women, with a mean age of $49 \pm 14$ years. The mean energy intake (kcal/day) was higher in men (2497) than women (2051), $p<0.001$. The mean daily caloric distribution was fat $(36.8 \%)$, proteins $(18.3 \%)$ and carbohydrates $(44.9 \%)$. Mean consumption of cholesterol is $313.2 \mathrm{mg} /$ day. According to Spanish Dietary Recommended Intakes (SDRI), in terms of quality fatty acids, this sample has a normal median ratio (MUFA+PUFA)/SFA (2.1) and by contrast has a low median ratio PUFA/SFA (0.4). As compared to SDRI, the intakes of patients assessed had deficit of (in $\%$ of patients): energy (55\%), fiber (76\%), folic acid (57\%), vitamin E (74\%), iodine $(83 \%)$, biotin $(88 \%)$, copper $(58 \%)$ and chlorine $(80 \%)$. In contrast, there is an excessive intake of: protein (93\%), vitamin C (82\%), vitamin A (54\%), vitamin B1 (67\%), vitamin B12 $(90 \%)$, iron $(68 \%)$, zinc $(61 \%)$, selenium $(61 \%)$, vitamin B2 $(71 \%)$, niacin $(87 \%)$, pantothenate $(52 \%)$, pyridoxine $(83 \%)$, phosphorus (92\%) and sodium (54\%). Our sample shows 5\%, $6 \%, 9 \%, 12 \%$ and $58 \%$ of patients only covered a quarter of the SDRI for energy, fiber, iodine, chlorine and biotin respectively. Conclusions: Energy and nutrient intakes of patients with HD deviate far from the recommendations. Inadequate diet consuming is a risk factor that may place patients with $\mathrm{HD}$ for developing malnutrition later. Identifying population groups of HD at risk of a nutritionally unbalanced diet will facilitate targeted intervention, necessary as part of integrated nutrition surveillance. Acknowledgements: We gratefully acknowledge to European Huntington's Disease Network for financial support to this work.

\section{DIFFUSIVITY OF THE CORTICAL SPINAL TRACT IN HUNTINGTON'S DISEASE}

Margherita Di Paola, Owen Robert Phillips, Cristina Sanchez-Castaneda, Francesca Elifani, Vittorio Maglione, Alba Di Pardo, Carlo Caltagirone, Umberto Sabatini, Ferdinando Squitieri

\section{Abstract.}

Introduction: Motor impairments are a critical feature of Huntington's disease (HD) and have been demonstrated in Presymptomatic HD (PreHD) subjects. Furthermore, white matter abnormalities have been shown in presymtomatic and symptomatic HD subjects using Magnetic Resonance Imaging (MRI), and Diffusion Tensor Imaging (DTI).

Objectives: To examine the crucial white matter motor tract that connects the primary motor cortex to the spinal cord, the cortical spinal tract (CST), using DTI tractography in HD.

Methods Subjects: (25 HD, 25 PreHD, 50 healthy controls). DTI data was acquired (3T Allegra for 3 repeats), using SE echo-planar imaging (TE/TR: 89/8500 ms, bandwidth: 2126 Hz/voxel, matrix: $128 \times 128,80$ axial slices, voxel size:
$1.8 \times 1.8 \times 1.8 \mathrm{~mm}) \quad$ with 30 isotropically distributed orientations for the diffusion sensitizing gradients at $a b$ value of $1000 \mathrm{~s} / \mathrm{mm}^{2}$ and $6 \mathrm{~b}=0$ images. DTI images were processed with FMRIB's Software Library. Tractography was done in TrackVis. Tractography was performed by manually drawing two regions of interest on each individuals fractional anisotropy color map. A general linear model was used to test for differences between groups with sex and age included as covariates. Correlations analysis was done between CAG repeat length, Disease Burden and UHDRS1 (motor assessment). Results: Tractography results showed decreased fractional anisotropy and increased axial and radial diffusivity in the CST of HD patients. PreHD and HD, CST FA, AD, and RD were correlated with CAG repeat length and Disease burden, as well as motor (UHDRS1I) assessment.

Conclusion: We have shown using DTI tractography, that the CST is impaired in HD patients. Furthermore, the tract is correlated with motor scores, underscoring the important functional role it plays in motor impairment in HD. CAG repeat length negatively affects the connectivity of the CST, suggesting a strong genetic component for structural connectivity.

\section{DNAJB6: A PEPTIDE CHAPERONE THAT DELAYS DISEASE IN THE HUNTINGTON R6/2 MOUSE MODEL}

\section{Harm H Kampinga}

Abstract. DNAJB6 is a member of the DNAJ family of chaperones that was found to have superior anti aggregation activity in cell, Drosophila and Xenopus models of Huntington's disease (1). We found that DNAJB6 prevents aggregation initiated by toxic fragments as generated during proteolysis of the huntingtin protein or as may arise due to alternative splicing of the huntington mRNA $(2,3)$. Transgenic mice expressing DNAJB6 in the brain were viable and showed no abnormalities up to 1 year of age. Crossing the DNAJB6 mice with R6/2 Huntington mice (200 CAG repeats) revealed that DNAJB6 strongly delayed aggregate formation in the brain. In addition, DNAJB6 $\times$ R6/2 mice showed slower decline in functional parameters (clasping, rotarod) and lived $23 \%$ longer than their R6/2 litter mates. Thus, DNAJB6 is the chaperone showing the largest protection against Huntington's disease reported to date. (1) Hageman et al., Mol. Cell. 37 (2010) 355-369. (2) Gillis et al., J. Biol. Chem (2013), in press (3) Mansson et al., submitted.

\section{DOES PRESYMPTOMATIC TESTING CHANGES AGE AT ONSET: A PROSPECTIVE STUDY IN HUNTINGTON DISEASE (PAON STUDY)}

Marcela Gargiulo, Marie-France Jutras, Sophie Tezenas du Montcel, Celine Jauffret, Sandra Benaich, Ariane Herson, Josué Feingold, Alexandra Durr

Abstract. In PAON, we studied prospectively the impact of presymptomatic testing (PT) on the onset of symptoms in 
Huntington disease (HD). The goal of our study was to estimate a possible test-effect on age at onset in carriers of the mutation who choose to know their genetic status. Does knowledge about being a carrier of the pathological mutation changes age at onset or onset modalities? In Paris, at the Salpêtriere University Hospital - Genetic Department, 1634 at-risk persons for HD requested genetic testing since 1992 and entered multistep and multidisciplinary care and counseling. We went back to those who received a test result before 2009, in order that their age at examination was reaching the mean expected onset of 40 years. Among them, 463 were non carriers and 302 carriers of an abnormal expanded CAG repeat. Detailed follow up examination was available for 208 individuals $(69 \%)$. We interviewed and examined additional 62 persons (PAON cohort), using a selfadministered questionnaires (to explore their carrier condition) and the UHDRS evaluation score. We estimated age at onset with the Langbehn formula (personal communication of Doug Langbehn and Clin Genet. 2004 Apr;65(4):267-77). Among the PAON cohort, there were 35 affected with UHDRS $>5$ (mean 18.3 +/- 11.9) and 27 unaffected (mean $2.9+/-1.8$ ). Surprisingly, age at onset observed was $6.0+/-8.7$ years earlier than onset calculated. Among those followed more regularly, 60 were affected (mean $26.1+/-18.3$ ) and had ages at onset $5.2+/-7.2$ years earlier than calculated. This did not differ significantly from a retrospective cohort of patients who did not have PT $(n=522)$ where age at onset was $3.6+/-9.6$ years earlier $(p=0.13$ and $p=0.12$, respectively). In conclusion, onset observed in a prospective cohort was earlier than that estimated based on CAG repeat length and age. This could be due to 1) a greater self-observance and a rising burden after PT, but the comparison with onset in patients without PT was not significant; 2) the fact, that the Langbehn formula underestimates onset. Importantly, for carriers of the mutation, knowledge about the genetic status after PT does not allow better awareness of the onset. Affected carriers did not always acknowledge symptoms related to the disease (44\%) and unaffected carriers did feel affected $(18.5 \%)$. A subjective anticipation (self-observation) of the onset was observed for both, affected (96\%) and unaffected carriers (70\%).

\section{DYSPHAGIA IN HUNINGTON'S DISEASE}

Natividad Mariscal, Esther Cubo Delgado, Jessica Rivadeneyra, Diana Armeso, Asunción Martínez Descalls, Spanish European HD Registry

\footnotetext{
Abstract.

Background: Dysphagia is a common complication of neurogenerative disorders, and a leading cause of death due to aspiration pneumonia. Little is known about the frequency and the characteristics of dysphagia in HD. Well-balanced strategies for treatment and prevention of dysphagia in HD are lacking.

Objetives: To determine the prevalence of dysphagia in HD. Methods: National, multicenter, observational, crosssectional study. Dysphagia was assessed using the questionnaire EAT-10 (eating assessment tool), caregiver burder using the SumaCare, functional capacity using the TFC score, disease severity using the Unified HD Rating Scale (UHDRS), and quality of life using the SF36.
}

Results: we present data from the first 128 patients included with $53,9 \%$ women, and mean age $48,89+14,16$, median TFC score of 6.5 (IQR 10.7), and median UHDRS motor score of 42 (IQR 53). One hundred and twenty three patients $(96 \%)$ the EAT-10. Dsyphagia was found in $45 . \%$ of the sample and equally present in men compared to women. Patients with dysphagia had higher UHDRS motor scores $(p<0.0001)$, cognitive scores $(p<0.0001)$, and lower TFC scores $(p<0.0001)$. There were not statiscal differences between patients with/without dysphagia in terms of age, caregive burden, and quality of life scores.

Conclusions: Dysphagia is very frequent in HD. Patients with higher motor and cognitive disturbances and lower functinoal capacity are at risk for dysphagia. Further strategies to identify dysphagia and to prevent aspiration pneumonia should be developped in HD clinics.

\section{DYSPHAGIA IN HUNTINGTON'S DISEASE: EVALUATION BY VIDEOFLUOROSCOPY}

\section{Anne-Wil Heemskerk, Berit Verbist, Raymund Roos}

\section{Abstract.}

Background: Huntington's disease (HD) patients suffer from dysphagia. Dysphagia bears a great risk for aspiration pneumonia, which is even the most frequently encountered primary cause of death. Little is known about the specific dysphagia features, such as the transit times of the four phases of ingestion, and the progression of symptoms during the three clinical stages of the disease. We investigated the specific features of dysphagia in HD with the aim to identify the swallowing problems in order to get a better insight into supportive treatments possibilities.

Methods: Forty-five HD patients (22 females, 23 male, mean age $53,7 \pm 11,8$ years), in the different stages of HD underwent a videofluoroscopy (VFS). Most patients $(81,6 \%)$ complained of swallowing problems. The video's were analysed and the clinical evaluation revealed problems in the oral, oropharyngeal and oesophageal phase. Further, duration measures (in sec) were calculated: oral transit time (OTT), pharyngeal transit time (PTT), oropharyngeal transit time (OPTT), pharyngeal delay time (PDT), pharyngeal passage time (PPT), and velopharyngeal closure time (VPCT).

Results: During the clinical evaluation, we found disturbances in the oral and oropharyngeal phase in $80 \%$ of the patients. Disturbances consisted of involuntary movements of the tongue and soft palate, residue in the valleculae and piriform sinus, and disturbed timing of the onset of swallowing. Swallowing abnormalities were seen in all three stages of the disease (stage I: 9/14, stage II: 10/14, stage III: $16 / 17$ patients). Aspiration was seen in $36,4 \%$ of the patients, but mostly in stage III patients (stage I: $3 / 14$, stage II: 4:14, stage III: 9/16). Conclusions: Swallowing abnormalities in HD are mostly due to oral- and oropharyngeal phase problems. Aspiration is seen in more than $1 / 3$ of the patients. Dysphagia features are present in all three clinical stages of the disease. Given the high rate of dysphagia and the early onset in the course of HD timely dysphagia management should be considered to prevent secondary complications. 


\section{EFFECTIVENESS OF A PHYSIOTHERAPY PROGRAMME, INCLUDING EXTERNAL CUES AND EXERCISES TO IMPROVE GAIT, BALANCE AND FUNCTIONAL INDEPENDENCE IN HUNTINGTON'S DISEASE}

Tamine Capato, Monica Haddad, Maria Elisa

Piemonte, Egberto Reis Barbosa

Abstract.

Objective: The aim of the study was to check the effectiveness of a physiotherapy programme including external cues and exercises to improve gait, balance and functional independence in Huntington's Disease(HD).

Background: HD progression causes movement cognition and behavior injuries. Although chorea is predominant the bradykinesia contributes to the loss of functional independence as it causes gait and balance disorders. Few studies investigate the effectiveness of rehabilitation strategies. Nevertheless, there are evidences that a physiotherapy treatment with attention strategies and external cues improve functional disturbances of the basal ganglia motor circuit. But, the mechanisms by which improvement occur remains inexplicable.

Methods: 30 HD patients divided on 3 groups, aged 45.91, were assessed by a blind examiner before and after 10 training sessions (once in a week during $45 \mathrm{~min}$ ) and after 60 days of the end of the training. Balance was assessed by Berg Balance Scale (BBS) and gait by Time UP and GO (TUG). Mobility was evaluated by Functional Independence Measurement Scale (FIM). Through interviews independence to AVDs was assessed; motor performance and cognition through UHDRS. The subjects should have HD diagnosis genetically confirmed and they were expected to understand tests and exercises sequences according to inclusion criteria. During the study period there wasn't medication changing. The first Group (I) done treatment programme comprised generalized exercises with external cues and was divided in three parts: 1 . Warming (10 minutes/stretching, trunk mobility and motor control); 2. Main Part (30 minutes/gait training with cues, balance and functional activities/attention strategies; 3. Relaxation (5 minutes/breathing exercises and trunk control). The second one (Group II) receives a book for to do exercises at home with their family. And the last one group (Group III) receives orientations and no exercises. Results showed a significant improvement after treatment, according to media score for the Group I of BBS $(p<0.01)$ TUG $(P<0.01)$ FMI $(P<0.01)$ and presents gait balance and mobility improvement. After 60 days there are scores maintenance for BBS and MIF. There weren't significant changes for groups II and III.

Conclusions: The physiotherapy programme proposed in this study is effective and connected to medicine seems to be effectiveness to minimize gait and balance disorders in HD and provides improvement in functional independence.

\section{EFFECTS AND MECHANISMS OF IMPAIRED LACTATE CLEARANCE IN HUNTINGTON'S DISEASE}

Signe Marie Borch Nielsen, Lisa Blanc Folmann, Anne Nørremølle, Maria Petersen, Bjørn Quistorff, Knud Josefsen, Lis Hasholt

\begin{abstract}
.
Introduction: Peripheral tissue changes are well documented in Huntington's disease (HD). We have previously shown reduced lactate clearance indicating impaired liver gluconeogenesis in HD patients and in R6/2 transgenic HD mice, correlating with reduced mRNA levels and enzyme activity of phosphoenolpyruvate carboxykinase (PEPCK), a rate-limiting enzyme in gluconeogenesis. The observed $19 \%$ reduction in PEPCK activity, however, may not be the sole cause of the changes in lactate clearance. As mitochondrial dysfunction has been proposed in $\mathrm{HD}$, and the initial processes of gluconeogenesis take place in the mitochondria, we investigated if reduced mitochondrial metabolism is an alternative explanation for the impaired gluconeogenesis.

Objectives: We set out to explore the time course of gluconeogenic impairment, the mechanisms causing it, and the physiological consequences.

Methods: Studies were performed on R6/2 and wt littermates, in vivo, and in isolated primary hepatocytes, liver homogenates and isolated liver mitochondria. We measured lactate clearance, blood glucose, cellular ATP level, mitochondrial respiratory chain activity, citrate synthase activity and transcription factor mRNA levels. Promoter assay was performed by co-transfection with HD exon 1 constructs and a PEPCK expression reporter system.

Results: Significantly impaired lactate clearance in vivo in 4 weeks old R6/2 mice indicate that these are early changes preceding the onset of HD symptoms. A direct effect of mutant huntingtin on the PEPCK promoter was not evident. An indirect effect on PEPCK expression via changes in mRNA level of the transcription factor genes Crebh, Mpk, Gcgr, Src-1, Sirt1 and Foxol in liver tissue could also be ruled out. Mitochondrial function was assessed in isolated liver mitochondria; no significant difference was observed, although we noted a general trend towards lower activities of respiratory chain complexes and citrate synthase activity in R6/2. Physiological effects of a reduced gluconeogenesis in the R6/2 mice was indicated by lower ATP levels in isolated primary hepatocytes as well as significantly lower blood glucose levels in vivo after prolonged fasting.

Conclusion: Our results do not give definite answers to the mechanism of HD-induced impairment of gluconeogenesis, but the physiological measurements indicate that the R6/2 mice, and maybe also the HD patients, have a reduced capacity for maintaining normal blood glucose level during fasting.
\end{abstract}

\section{ENROLL-HD: A GLOBAL OBSERVATIONAL STUDY ENABLING CLINICAL RESEARCH IN HUNTINGTON'S DISEASE}

Tiago Alexandre Mestre, Joe Giuliano,

Olivia J. Handley, Bernhard Landwehrmeyer, Cristina Sampaio

\footnotetext{
Abstract.

Introduction: Huntington's disease (HD) is a familial progressive neurodegenerative disease without cure. There is a need to find better treatments, namely, interventions that can modify the course of the disease. An observational study in HD will increase the knowledge of its natural history and
} 
provide a dynamic platform of HD clinical centers for future clinical studies.

Objective: The primary aim of Enroll-HD is to enable clinical research to accelerate the development of new therapies for HD. The secondary aims are to understand the natural history of HD and to inform clinical care and improve health outcomes.

Methods: Enroll-HD is an observational study collecting clinical data and bio-specimens, with evaluations at baseline and at annual follow-up visits. Enroll-HD is an open-end study. Eligible participants are HD gene expansion mutation carriers (symptomatic or asymptomatic), "at-risk" family members with unknown mutation status. Controls include family members who are HD gene expansion negative and non-blood related healthy individuals. The study is multinational multicenter with four recruitment regions (North America, Europe, Latin America and Australasia) and a total of 265 participating centers. The aim is to recruit one third of the HD affected population in each study region. A data monitoring process ensures data is complete, accurate, consistent and compliant with protocol. Preliminary results: (June 2013) Enroll-HD has recruited 754 participants in 29 centers $(28$ centres in North America; 1 centre in Latin America). $442(58 \%)$ are HD gene expansion mutation carriers, of which $328(43 \%)$ are symptomatic. $179(24 \%)$ participants are family controls. By the end of 2013, the remaining world regions will have started recruitment. The current European-based Registry Study (10-year observational study for HD; 10,000 participants) will initiate data migration into Enroll-HD for all consenting participants. The transition period is scheduled from then end of 2013 until 2014.

\section{EUROPEAN HUNTINGTON'S DISEASE NETWORK - REGISTRY: CURRENT STATUS}

Olivia Handley, Ruth Fullam, Katrin Barth, Tim McLean, Jean-Marc Burgunder, Bernhard Landwehrmeyer, the Registry Steering Committee and the EHDN Registry Investigators

\footnotetext{
Abstract.

Introduction: The European Huntington's Disease Network's (EHDN) REGISTRY is an observational, prospective, longitudinal, multi-national, multi-centre cohort study of Huntington's disease (HD). It is the largest study of HD to date, and collects valuable phenotypical data and biospecimens on at risk, premanifest or manifest HD individuals, nonexpansion mutation carriers and control participants.

Objectives: R. was conceived to establish a defined Europeanbased HD population to expedite high-quality research for the improvement of knowledge of this disease and the development of HD specific tools, to support the work towards discovering and developing therapies to delay or slow HD.

Methods: R. follows a prospective study design with participants attending annual visits. Pseudonymised clinical data are captured online in a webportal (www.euro-hd.net) and stored in a central database in Ulm, Germany; biospecimens are processed and stored centrally at BioRep, Italy. All data are subject to regular quality control checks for plausibility and accuracy. To date five sub-studies have been activated.
}

Results: R. data have been collected in 17 European countries. Over 11000 participants contributed phenotypical data for the evaluation of the natural history and progression of $\mathrm{HD}$, of these more than 8000 donated biosamples for genetic modifier and biomarker studies. On average each month 150 new participants are enrolled into R., 350 follow-up visits are conducted and 400 biospecimens are submitted. A summary of the key study metrics including demographics and disease stage of the REGISTRY cohort and an overview of sub-study enrolment figures are presented. The Physiotherapy sub-study met its recruitment target $(n=80)$, the Juvenile Huntington's Disease sub-study exceeded its recruitment target and is ongoing ( $n=72$ to date), the Quality of Life $(n=192)$ and Lifestyle $(n=178)$ sub-studies are ongoing and the Behavioural Phenotype sub-study recruitment has just started. Conclusions: REGISTRY has successfully established a well-characterised European-based HD cohort. The study has proven to expedite the implementation of clinical trials in HD, substudies and regular data collection were facilitated. In 2013 REGISTRY will begin its transition into Enroll-HD, the first global observational study on HD (www.enroll-hd.org), and will subsequently formally end. The current database will be retained as a legacy database and made accessible for further studies.

\section{FUNCTIONAL CHANGES DURING A WORKING MEMORY TASK IN HUNTINGTON'S DISEASE: 30 MONTH LONGITUDINAL DATA FROM THE IMAGE- HD STUDY}

Govinda R Poudel, Marcus A Gray, Juan F Domínguez D, Louisa Salmon, Andrew Churchyard, Phyllis Chua, Beth Borowsky, Julie C Stout, Gary F Egan, Nellie Georgiou-Karistianis

\section{Abstract.}

Introduction: Cross-sectional functional MRI (fMRI) studies have shown that change in functional activation patterns in Huntington's disease (HD) may be dependent on stage of disease. There have, however, been no longitudinal fMRI studies investigating change in activation patterns over more than two time points. Here we report a 30 month fMRI investigation of working memory (WM) performance in premanifest HD (pre-HD) and symptomatic HD (symp-HD) using data from the Australian based IMAGE-HD study.

Objectives: To document 30 month longitudinal change in fMRI activity during a WM task in pre-HD and symp-HD individuals as part of an investigation of mechanisms of functional compensation.

Methods: The current study involved 22 pre-HD, 11 symp$\mathrm{HD}$ and 20 age-matched (to pre-HD) healthy controls. Participants underwent fMRI testing at three time points over 30 months (baseline, 18 and 30 months). We used an N-BACK paradigm consisting of three conditions (0-BACK, 1-BACK and 2-BACK). We analysed fMRI activity over time during 1-BACK WM as well as functional connectivity over time between the dorsolateral prefrontal cortex (DLPFC) and caudate.

Results: We found significant group differences in longitudinal change in fMRI activity during 1-BACK and 2-BACK 
performance. Compared with controls, the pre-HD group showed increased activity in the left DLPFC and medial frontal cortex during 1-BACK, and in bilateral caudate, putamen, and temporal cortex during 2-BACK. Only the pre-HD group showed longitudinal decrease in functional connectivity between left DLPFC and caudate during both WM loads.

Conclusion: We provide the first evidence for a differential pattern of longitudinal change during premanifest and symptomatic stages of HD. The ability to maintain and enhance activation in the DLPFC may represent an early compensatory response during the premanifest stage of the disease.

\section{FUNCTIONAL HYPERCOMPENSATION IN PRODROMAL HUNTINGTON'S DISEASE}

Patrick Weydt, Kathrin Malejko, Sigurd Süssmuth, Georg Grön, G. Bernhard Landwehrmeyer, Birgi Abler

\begin{abstract}
.
Objective: Functional hypercompensation demonstrated as rescue mechanism to offset neuronal loss in early Alzheimer's disease may also occur in other adult-onset neurodegenerative diseases. With its genetic determination, Huntington's disease (HD) provides the opportunity to investigate this mechanism before full symptom manifestation. In HD neurodegeneration typically initiates in the dorsal striatum, successively affecting ventral striatal areas. Investigating carriers of the HD mutation with evident dorsal, but only minimal or no ventral striatal atrophy, we expected to find evidence for hypercompensation of ventral striatal functioning.

Methods: We investigated 14 mutation carriers (MC) at an average of 19.6 years (SD 11.6) before onset of the illness and 18 matched control subjects. Participants underwent structural T1 magnetic resonance imaging (MRI) and functional MRI during a reward task that probes ventral striatal functioning. Motor functioning and attention were assessed with reaction time (RT) tasks.

Results: Structural images confirmed a specific decrease of dorsal striatal but only marginal ventral striatal volume in $\mathrm{MC}$ as compared to control subjects paralleling prolonged $\mathrm{RT}$ in the motor response tasks. RT in the reward task during fMRI scanning were unimpaired. Comparing reward-related fMRI signaling in the bilateral ventral striatum and in bilateral orbitofrontal cortex/anterior insula, as another region sensitive to reward processing, we found evidence for increased signaling in $\mathrm{MC}$ in all regions of interest.

Conclusions: We provide first evidence for the concept of functional hypercompensation in premanifest HD. This suggests a general defense mechanism in neurodegeneration and may also offer a rationale for dopaminergic pharmacotherapy in early stages of HD.
\end{abstract}

\section{GASTRIC BIOPSIES REVEAL POSSIBLE GASTRIC DYSFUNCTION IN HUNTINGTON'S DISEASE}

Andrew C. McCourt, Gill Bates, Maria Björkqvist, Oliver W Quarrell

\begin{abstract}
.
Introduction: Weight loss is an important complication of Huntington's disease (HD). It correlates with disease progression and affects the quality of life of HD patients, suggesting that it could be a valuable target for therapeutic intervention. The mechanism for weight loss in HD is not entirely known. Mutant huntingtin is expressed along the gastrointestinal (GI) tract and the GI tract of HD mice has been shown to be affected. HD mice exhibit reduction of neuropeptides, decreased mucosal thickness and villus length, alongside gut motility impairment, resulting in malabsorption of nutrients.
\end{abstract}

Objectives: Therefore, we investigated gastric mucosal biopsies of patients with HD, looking for abnormalities of mucosal cells.

Method: Gastric biopsies were obtained upon a clinical decision to insert a feeding tube; control samples were obtained from patients who do not have neurodegenerative disease and have had normal gastric biopsies. Cell types were investigated using immunohistochemistry.

Results: In order to investigate possible histological differences related to gastric acid production, we evaluated the number of acid producing parietal cells, as well as gastrin producing cells (the endocrine cell controlling parietal cell function). Here, we describe reduction of gastrin, and no change in parietal cell number. This is in line with previous HD mouse studies showing reduction of GI tract neuropeptides. This study was supported by a seed fund from EHDN.

\section{HDAC4 REDUCTION: A NOVEL THERAPEUTIC STRATEGY TO TARGET CYTOPLASMIC HUNTINGTIN AND AMELIORATE NEURODEGENERATION}

Michal Mielcarek, Christian Landles, Andreas Weiss, Amyaouch Bradaia, Tamara Seredenina,

Linda Inuabasi, Georgina F Osborne, Kristian Wadel, Chrystelle Touller, Rachel Butler, Janette Robertson, Sophie A Franklin, Donna L Smith, Larry Park, Paul A Marks

Abstract. Histone deacetylase (HDAC) 4 is a transcriptional repressor that contains a glutamine rich domain. We hypothesised that it may be involved in the molecular pathogenesis of Huntington's disease (HD), a protein folding neurodegenerative disorder caused by an aggregation-prone polyglutamine expansion in the huntingtin protein. We found that HDAC4 associates with huntingtin in a polyglutaminelength dependent manner and co-localises with cytoplasmic inclusions. We show that HDAC4 reduction delayed cytoplasmic aggregate formation, restored Bdnf transcript levels and rescued neuronal and cortico-striatal synaptic function in HD mouse models. This was accompanied by an improvement in motor co-ordination, neurological phenotypes and increased lifespan. Surprisingly, HDAC4 reduction had no effect on global transcriptional dysfunction and did not modulate nuclear huntingtin aggregation. Our results define a crucial role for the cytoplasmic aggregation process in the molecular pathology of HD. HDAC4 reduction presents a novel strategy for targeting huntingtin aggregation which may be amenable to small molecule therapeutics. 


\section{HELP OR HINDRANCE: YOUNG ADULTS' EXPERIENCES OF PREDICTIVE TESTING FOR HUNTINGTON'S DISEASE IN THE TRANSITION FROM ADOLESCENCE TO ADULTHOOD}

Karen Forrest Keenan, Lorna McKee,
Zosia Miedzybrodzka

\begin{abstract}
.
Background: Clinical observations indicate that growing numbers of young adults are requesting predictive testing for $\mathrm{HD}$, yet there is little research in this area, particularly outcomes of relevance to adolescent psychosocial development. The aim of this study was to explore young adult's experiences of predictive testing for $\mathrm{HD}$, the impact of their result, their opinions of the testing process and to identify gaps in information or support.

Methods: In-depth qualitative interviews were conducted with young adults who sought predictive testing for HD from nationally funded Scottish Genetics Services. Participants were recruited through the Grampian Genetics Service or Scottish Huntington's Association. 11 participants aged 17 26 were recruited (seven under age 20), and two partners. All were female. Pre- and post-test interviews were conducted where possible ( $n=8$ pre-test).

Results: A preliminary thematic analysis suggests three main testing experiences, regardless of test result. Testing may be: (1) a journey of empowerment, (2) an ambivalent process or (3) a poor experience. Some participants reported unanticipated changes in family communication which they had difficulty in coming to terms with. Gaps in emotional support were also highlighted, particularly for those living with an affected parent.

Conclusions: Predictive testing for HD may assist some young adults in their transition from adolescence to adulthood. However the test results and/or the test process may also have a detrimental impact particular to this stage of development, for example, establishing personal identity \& peer relations, developing intimate relationships and separation from parents. Strategies to improve the testing experience for young adults will be discussed.
\end{abstract}

\section{HUMAN STRIATUM: NITRERGIC NEURONAL DENSITY OF CAUDATE, PUTAMEN AND NUCLEUS ACUMBENS IN NORMAL AGING}

Bruno Lopes dos Santos, Elaine Aparecida Del Bel, Vitor Tumas

\footnotetext{
Abstract.

Introduction: The Huntington's disease (HD) is an autossomic dominant neurodegenerative disorder, and the most striking neuropathological feature is the progressive striatal atrophy, with marked neuronal loss and astroglial proliferation. For unknown reasons, the neuron loss spares a specific aspiny interneuron type which contains nitric oxide (NO), detected by the histochemical reaction for nicotinamide adenine dinucleotide phosphate diaphorase (NADPHd). The growing importance of the nitrergic system over motor control
}

has been highlighted in the last years, with possible therapeutic implications for movement disorders. It is not known the role of normal aging over this striatal interneuron type in humans. Objectives: To investigate the role of normal aging over the nitrergic neuronal density in striatum.

Methods: The striatum (caudate, putamen and nucleus acumbens) of 20 subjects without neurologic or psychiatric diseases was evaluated by histological analysis of sections stained with NADPHd histochemistry, and the nitrergic neuronal density was compared between young adults and old individuals, and between topographic and functional subdivisions.

Results: The NADPHd-positive neurons represented $2 \%$ of total neuronal density in striatum. There were no differences on nitrergic neuronal density between young adults and old subjects in caudate, putamen and nucleus acumbens. The NADPHd-positive neuronal density was higher in the posterior levels of caudate and putamen, and the striatal area associated with the limbic regions (ventral putamen) had the highest NO-related cell density.

Conclusions: The normal human aging do not alter the striatal nitrergic neuronal density, the limbic striatum has the highest NADPH-positive neuronal population, and there is a physiologic anterior-posterior distribution gradient of these aspiny interneurons in striatum.

\section{HUNTINGTON - THE STORY OF A GENE IN SEARCH OF A BETTER FUTURE}

\section{Elena Cattaneo}

Abstract. Over the past twenty years, a number of studies have revealed important aspects of the function, origin and evolution of the normal Huntington's Disease gene. We now know that the protein product of this gene named huntingtin (HTT) is critical during several phases of life and growing evidence shows that impairment of its normal functions by an abnormally long polyglutamine (polyQ) tract can contribute to the pathogenesis of HD. We also know that HTT is a very ancient gene that can be traced through evolution to 800 million years ago. Here I will review the story of this gene all throughout the millennia. Telling the story of this ancient gene will not change, for the moment, the course of this tragic disease. But the need to understand more bring us together as we all have that ancient gene.

\section{HUNTINGTON AND DANCE 10 YEARS JUBILEE OF CONTEMPORARY DANCE WORKSHOPS FOR PEOPLE AFFECTED BY HUNTINGTON'S DISEASE}

\section{Philippe Chehere}

Abstract. This year is the 10th anniversary of a project that we called "H like Huntington, D like Disease, H like Hospital, D like Dance", an artistic project for and with people affected by Huntington's Disease (patients, family, nurses, caregivers). The dance workshops take place in turns in a hospital amphitheatre, in a contemporary dance studio and, at participants' requests, at their home,in Paris. Methods/Approach 
The content provides participant with a space for relaxation, a space to develop consciousness of their own body and the body of others, and, through creative work, to experiment with different ways to move one's body, by using an approach that works with the imaginary, with poetry, inventiveness and explorations of our multiple sensory modalities. Importantly however, our approach is artistic and not therapeutic; We do not intervene as clinical specialists or therapists but as dancers. The experience of the dance workshops at the hospital reveals to what point dance practice can stimulate new ways of relating to oneself and relating to others. We found that releasing their body evoked the release of participants' communication. Contemporary dance creates bodily states that favour new perceptions and room for poetry, through improvisation and contact dance. Further, we observe how the body posture can be modulated by means of our danse expertise and how in modifying body tonus we change our empathic stance, even in the face of unvoluntary movements that mark HD. Finally, our work investigates the core role of movements in HD: Through our work we create new ways of being together with the other, dansomania becomes a motor of creativity instead of a burden. Outcome In 2013-2014, we are going to establish a protocol of scientific research in collaboration with specialized doctors in order to evaluate the influence of the artistic pratice of contemporary dance for thouse patientes suffering from Huntington Disease. Alltogether, for 10 years now, we have gathered enormous evidence of the positive and supportive effect of the "Hospital Danse" project on patients and their relatives and caregivers. These experiences are captured in a 15 minutes documentary that we compiled and would like to present at this years WCHD network conference. Support by doctors : A.Dûrr, M.Gargiulo, France.

\section{HUNTINGTON DISEASE AND HUNTINGTON DISEASE-LIKE IN A CASE SERIES FROM BRAZIL}

Laura Bannach Jardim, Aline FD Souza, Gabriel V Furtado, Tailise C Gheno, Raphael M Castilhos, Alisson L. da Silva, Fernando R Vargas, MariaAngelica FD Lima, Karina C Donis, Orlando Barsottini, José L Pedroso, Clecio Godeiro-Junior, Diego Salarini, Elian

\begin{abstract}
.
Although the most important genetic cause of adult-onset chorea and cognitive decline is Huntington's disease (HD), other genetic diseases cause HD-like phenotypes, with different frequencies among populations. Aims: to identify the relative frequency of HD and HDL disorders (HDL1, HDL2, SCA2, SCA17, DRPLA, benign hereditary chorea, neuroferritinopathy and choreoacanthocytosis), in a series of Brazilian families. Methods: patients were recruited in 7 neurogenetic centers by the presence of 2 of the following items (at least one major); major: chorea, cognitive decline; others: Parkinsonism, dystonia, and AD inheritance. Clinical data was obtained, and molecular studies of HTT, ATXN2, TBP, ATN1, JPH3, PRNP, FTL, NKX2-1/TITF1 and VPS13A genes were performed. Results: 104 families were ascertained from 2001 to 2012: 71 families lived in the South, 25 in the
\end{abstract}

Southeast and 8 in the Northeast region. There were $93 \mathrm{HD}, 4$ HDL2 and 1 SCA2 families. Six families remained without a molecular diagnosis. Twelve of the 104 index cases did not have a family history: 10 with HD and two without a molecular diagnosis (ns). Clinical characteristics were similar between $\mathrm{HD}$ and non-HD cases. In HD, the median of expanded (CAG) n (range) was 44 (40-81) units; R2 between expanded HTT and AO was 0.55 ( $p=0.0001$, Pearson). HDL2 was found in Rio de Janeiro (2/9 families) and Rio Grande do Sul states (2/68 families). Discussion: a systematic diagnostic strategy detected HD in $89.4 \%$, HDL2 in $3.8 \%$ and SCA2 in $0.9 \%$ of 104 Brazilian families. There were no cases of HDL1, SCA17, DRPLA, neuroferritinopathy, choreoacanthocytosis or benign hereditary chorea. Only six families $(5.8 \%)$ remained without molecular diagnosis, much less than in other series.

\section{HUNTINGTON DISEASE: CLINICAL AND MOLECULAR FEATURES OF A CASE SERIES FROM BRAZIL AND ARGENTINA}

Raphael Machado de Castilhos, Alisson L da SIlva, Patrícia S Bopsin, Aline FD Souza, Gabriel V Furtado, Tailise C Gheno, Fernando R Vargas, Maria-Angélica FD LIma, Karina C Donis, Orlando Barsottini, José L Pedroso, Clécio Godeiro-Junior, Diego Salarini

\section{Abstract.}

Aims: to describe the molecular and clinical features of a case series of Brazilian and Argentinian individuals with Huntington's disease (HD), with focus on geographical and on CAGexp transmission characteristics.

Methods: patients were recruited in 8 neurogenetic centers by the presence of 2 of the following items (at least one major); major: chorea, cognitive decline; others: parkinsonism, dystonia, and AD inheritance. Molecular and clinical data were obtained. Pedigrees with more than one individual examined were analyzed for intergenerational instability.

Results: 137 patients $(71$ men) from families were diagnosed. Eight patients lived in Argentina, whereas 129 were Brazilian individuals living in South (S; 80 patients), Southeast (Se; 36) and Northeast regions (Ne; 13). Among Brazilians, the median (range) ages of onset (AO) were of 39.5 (6 to 65), 40 (15 to 64) and 29.5 (7 to 55) years of age, whereas the median (range) CAGexp were of 44 (39-81), 44 (40-60) and 45 (4059 ) in $\mathrm{S}, \mathrm{Se}$ and Ne regions (ns, Kruskal-Wallis). There was a strong correlation between AO and CAGexp $\left(r^{2}=0.549\right.$, $p<0.0001$, Pearson) in the overall sample. The mean \pm sd of normal CAG alleles in trans were $17.9 \pm 2.9,17.6 \pm 3.2$ and $20.8 \pm 5$ in $\mathrm{S}$, Se and Ne regions $(p<0.007$, ANOVA). Five unrelated HD patients had intermediate alleles in trans (range: 27 to 30); three of them lived in Ne region. The mean \pm sd AO according to the genotypes Normal/Fully Expanded and Intermediate/Fully Expanded were $38 \pm 11$ and $30 \pm 18$ $(p=0.11, \mathrm{~T}$ test). The mean (range) of intergenerational gradient in the AO $(n=16)$ and CAGexp $(n=4)$ were $10(-8$ to 30) years and 7.25 ( -1 to 30$)$ CAGn. In the 20 sibships studied, the mean (range) of $\mathrm{AO}$ and CAGexp gradient between older to younger sib were 3 ( -9 to 19 ) years and 1.7 ( -5 to 7 ) CAGexp ( $p=0.082$, ANOVA). Chorea, cognitive decline, ataxia and ocular movement abnormalities were seen, 
respectively, in $95.1 \%, 80.5 \%, 80.2 \%$ and in $33.3 \%$ of patients. Parkinsonian rigidity was associated with earlier AO ( $p=0.019$,Spearman). Discussion: we confirm several finding widely described in literature. We found some trends pointing to the presence of longer normal alleles in Ne region of Brazil, what can be related to relatively large number of HD patients from that region carrying intermediate alleles in trans. Even though the number of patients studied was too small to draw any conclusion, there is a possibility of a diverse epidemiology of Huntington's disease within Brazil.

\section{HUNTINGTON ZULIA PROGRAM (HZP) IN VENEZUELA: ACHIEVEMENTS AND CHALLENGES.}

Ernesto Solís Añez, Wilmer Delgado, Gilda Pérez, Lilian González, Lennie Pineda, Olmedo Ferrer, Carlos Chavez, William Zabala, José Miguel Quintero, Zulay Finol

\begin{abstract}
.
Introduction: Zulia State in Venezuela has the communities with the highest prevalence of Huntington disease (HD) reported around the world. HZP have been developed since 2007 by the Genetic Research Institute (GRI) of the University of Zulia with the aim of decrease the prevalence of the disease and improve the quality of life of the affected families through the study of epidemiologic and socio-economical features, standardization of the genetic test, multidisciplinary medical attention, scientific collaborative research, support to patient organizations, educational speaks and Govern and public sensibilization.
\end{abstract}

Objective: to summarize the data of the HZP.

Methods: medical records, census, family tab of health of the Venezuelan Health Ministry, Graffar modified scale.

Results: Our data from Zulia State have been divided in: 1. GRI (patients from the whole Zulia state since 2007): 62 patients, $52.7 \%$ were male, mean age (MA) of 46.9 years, mean CAG expansion of $46.33 \pm 4.9$ and mean of age of onset (MAO) of $39.14 \pm 11.97$ years with significant negative correlation $(r=-0.783 ; P<0.0001)$. Most of the patient $(67 \%)$ were born in Maracaibo (capital city of Zulia). $54.4 \%$ of the patient were in the middle stage of the disease. 2. San Luis Community (SLC): total of families: 490; with history of chorea $(33.03 \%)$ ) 45 patients, prevalence of $2.540 / 100.000$. $57.7 \%$ were female, MA: 33.8 years. Denial of HD: $53.8 \%$, MAO: 28.35 years, suicidal ideation: $23.1 \%$ with suicidal attempt: $66.7 \%$. $42 \%$ of the patient were in the middle stage of the disease. Patients in other nearby communities: 27 Significant differences $(p<0.0001)$ in socioeconomical levels between families with no chorea history, where dominat levels were III $(23.8 \%)$ and IV (55.7\%), and families with history of chorea where levels IV (56\%) and V (37.3\%) (relative/extreme poverty) were dominant.. 3. Barranquitas Community: 102 patients, average age: 38.9 years old with socio-economical features similar to SLC. Ratio of patient registered to another family member affected and family member at $50 \%$ risk were 1:1.6 and 1:7 respectively in the overall data, so our estimated population of patients is at least of 378 , with 2.646 individuals at $50 \%$ risk and a minimum prevalence estimated in Zulia State of 10.2/100.000.
Conclusion: HZP is a local plataform that is generating important data for scientific research and medical and social assistance to the communities affected with HD in Venezuela

\section{HUNTINGTON'S DISEASE AND SENSORINEURAL HEARING LOSS}

Paulo Victor Sgobbi de Souza, José Luiz Pedroso, Wladimir Bocca Vieira de Rezende Pinto, Maria Luiza Saraiva-Pereira, Marcus Vinicius Cristino Albuquerque, Laura Bannach Jardim, Orlando Graziani Povoas Barsottini

\section{Abstract:}

Objectives: To describe clinical characteristics of a Brazilian family with Huntington's disease (HD) and sensorineural hearing loss, with focus on phenotypic variation among subjects affected, highlighting sensorineural hearing loss as a major manifestation.

Methods: Three siblings were evaluated at our clinic and genetic testing for HD was performed.

Results: Sibling-I: a 46-year-old man, presented with a 5 -year-history of rest hand tremor, progressive psychomotor agitation, gait instability and dysarthria. He also had severe bilateral progressive hearing loss. Sibling-II: a 43-year-old man, presented with a 10-year-history of gait instability, frequent falls, moderate bilateral hearing loss, and a 5-yearhistory of insomnia, psychomotor agitation, chorea and dysmetria. Sibling-III: a 55-year-old man, presented with a 5-year-history of hand tremor, gait instability, slurred speech, slowed ocular sacades, mild chorea and non-progressive hearing loss. Genetic testing was positive for HD in all three patients, and audiometry disclosed sensorineural hearing loss. Discussion: It is well established that there is an anomalous pattern of activation in auditory stimulation processing in HD patients, mainly in late-stage clinical disease. However, deafness has rarely been described in HD patients. It is hypothesized that mutant huntingtine supresses brain-type creatine kinase expression and elevates reactive oxygen species in cochlea hair cells in HD. Although we did not exclude the possibility of another cause for hearing loss in these three sibs, this pedigree might be one of the first ones where deafness seems to be linked to HD phenotype. We describe a large family with HD in which sensorineural hearing loss is a major and first manifestation of the disease.

\section{INSULIN SENSITIVITY AND EARLY-PHASE INSULIN SECRETION IN NORMOGLYCEMIC PATIENTS WITH HUNTINGTON'S DISEASE}

Giuseppe De Michele, Cinzia Valeria Russo, Elena Salvatore, Francesco Saccà, Tecla Tucci, Carlo Rinaldi, Pierpaolo Sorrentino, Marco Massarelli, Luigi Di Maio

Abstract.

Background: Huntington's Disease (HD) is an autosomal dominant neurodegenerative disorder caused by an expanded CAG repeat in exon 1 of the HTT gene. Apart from 
neurological impairment, there is increasing evidence pointing towards an early involvement of the endocrine system in HD. Recent studies, conducted to investigate the association with increased risk of diabetes mellitus and with impairment in insulin sensitivity and secretion in HD patients, led to contradictory results.

Objective: To investigate glucose homeostasis in HD, we evaluated insulin release and sensitivity by performing an oral glucose-tolerance test (oGTT).

Patients and Methods: Twenty-eight consecutive patients with HD and 28 healthy controls were matched for age, sex, and BMI. Diagnosis of HD was confirmed in all patients by analysis for the CAG repeat expansion. Clinical assessment of patients was performed using the Unified Huntington's Disease Rating Scale (UHDRS) motor section and the Total Function Capacity (TFC). Basal metabolic and endocrine investigations and a 2-hour 75-g oGTT were performed. We used the homeostasis model assessment of insulin resistance (HOMA-IR) as index of insulin sensitivity. Insulin secretion was determined by the insulinogenic index.

Results: HD patients did not differ from the control group with respect to fasting plasma glucose level, insulin sensitivity and secretion. CAG expansion size, disease stage and duration, or BMI did not influence HOMA-IR and insulinogenic index. HD patients showed lower serum glucose $(-19 \%)$ and insulin levels $(-48 \%) 30$ min after oral glucose load and higher serum insulin levels at $90(+132 \%)$ and 120 $\min (+380 \%)$. Conclusions: Our data do not favors the hypothesis of an increased risk of diabetes among HD patients although they show peculiar abnormalities of glucose regulation with a flat glucose curve and delayed insulin peak after oral glucose load.

\section{INTRANASAL OXYTOCIN INCREASES AMYGDALA RESPONSE TO EMOTIONAL CUES IN PATIENTS WITH HUNTINGTON'S DISEASE}

Izelle Labuschagne, Govinda Poudel, Catarina Kordaschia, Nellie Georgiou-Karistianis, Qi-zhu Wu, Andrew Churchyard, Julie C. Stout

\footnotetext{
Abstract.

Introduction: Huntington's disease (HD) is consistently associated with deficits in facial expressions of emotion which have been linked to amygdala dysfunction. It is now known that the neuropeptide, oxytocin, plays an important role in modulating brain-related responses to the perception of emotional stimuli, such as facial expressions, in healthy and clinical groups. However, the effects of oxytocin administration have not been examined in a neurodegenerative population. Evidence from a single study suggests that HD patients have a $45 \%$ reduction in oxytocin-expressing neurons in the brain.

Objectives: In a randomised double-blind placebo-controlled study, we examined whether oxytocin, when administered intranasally to HD patients, has beneficial effects on amygdala response to emotional face stimuli using functional magnetic resonance (MR) imaging. In a region specific approach, we examined overall amygdala effects as well as effects within the medial, dorsal and ventral subregions of the amygdala.
}

Methods: Our participant sample so far consisted of 4 presymptomatic HD patients and 6 controls, all right-handed males, studied at two visits. At each visit, participants received either an acute dose of oxytocin or placebo nasal spray. Participants then performed an emotional face matching task involving trios of Ekman faces and trios of shapes in the MR scanner.

Results: Compared to controls, patients with HD hyperactivated the amygdala to negative, but not positive, emotional faces in bilateral dorsal, right medial and right ventral amygdala under placebo treatment. Within the HD group, an acute dose of intranasal oxytocin increased the amygdala activity in response to negative emotions in bilateral dorsal and ventral amygdala, and to positive emotions in right medial and bilateral ventral amygdala. In controls, we did not observe oxytocin effects, compared to placebo, in the amygdala.

Conclusions: Our results so far indicate a hyperactive amygdala response to negative emotional faces in HD. Acute doses of oxytocin modulated subregional amygdala-related emotional responses in HD but not in controls. The findings suggest that it may be worthwhile to explore a therapeutic approach related to this oxytocin effect in HD.

\section{INVESTIGATION OF THE METABOTROPIC RECEPTOR 5 ROLE IN HYPERKINESIS USING A MICE MODEL OF HUNTINGTON'S DISEASE}

Isabella Monteiro Guimarães, Jéssica Mabelle Souza, Rita G. W. Pires, Stephen S. G. Ferguson, Fabíola Mara Ribeiro

\begin{abstract}
.
Objective: Investigate whether mGluR5 could play a role in HD related hyperkinetic symptoms. Introduction: Huntington's disease (HD) is an autosomal-dominant neurodegenerative disorder caused by a progressive neuronal cell loss in the cortex and striatum, which leads to hyperkinesias, dementia and death. We have recently demonstrated that the metabotropic glutamate receptor 5 (mGluR5), which is coupled to Gaq, might have a role in HD pathology. As a number of reports have demonstrated that mGluR5 is involved in hyperkinetic movements, we decided to investigate whether mGluR5 has a role in HD related hyperkinesias.
\end{abstract}

Methods: HdhQ20/Q20/mGluR5+/+, HdhQ20/Q20/ mGluR5-/-, HdhQ111/Q111/mGluR5+/+, HdhQ111/Q111/ mGluR5-/- mice were inserted into the open field apparatus and locomotor activity was measured as distance. A microarray assay was performed to determine protein expression levels, which were confirmed by qPCR.

Results: We observed that mGluR5-/-, as well as HdhQ20/ Q20/mGluR5-/- mice, exhibited much higher levels of locomotor activity than mGluR5+/+ mice. However, HdhQ111/Q111/mGluR5-/- mice did not exhibit increased locomotor activity, indicating that there is an interaction between mGluR5-mediated locomotion alterations and HDrelated dyskinesia. To investigate the mechanism underlying these locomotion alterations, we have performed a microarray assay. The expression of a number of proteins involved in movement regulation was altered in HdhQ111/Q111/ 
mGluR5-/- mice, as compared to HdhQ20/Q20/mGluR5-/mice. Such expression alterations were confirmed by qPCR The qPCR experiments confirmed that the mRNA transcript levels of dynein heavy chain, dynactin 3 and dynein light chain-6 are altered in HdhQ111/Q111/mGluR5-/- mice, as compared to HdhQ20/Q20/mGluR5-/- mice.

Conclusion: The results indicate that mGluR5 might play a role in HD diskynesia. Moreover, our microarray/qPCR data suggest that the mGluR5-related movement alterations observed in the HD mouse model might be due to modifications in protein expression levels.

\section{IRON CONTENT IN THE CORTICAL SPINAL TRACT IN HUNTINGTON'S DISEASE}

Owen Phillips, Cristina Sanchez-Castaneda, Francesca Elifani, Vittorio Maglione, Alba Di Pardo,

Carlo Caltagirone, Ferdinando Squitieri,

Umberto Sabatini, Margherita Di Paola

\begin{abstract}
.
Introduction: The progressive motor impairment is critical in Huntington's disease (HD) and subtly develops since before clearly manifest symptoms appear. For instance, the integrity of white matter connections between primary motor cortex and spinal cord, the cortical spinal tract (CST), which is involved in motor function, merits particular attention Objectives Higher iron concentration in subcortical gray matter structures is documented in HD. Here we wanted to investigate the iron content in the cortical spinal tract in HD, using Transverse Relations Rate (R2*), which is an iron sensitive imaging.
\end{abstract}

Methods: Subjects groups included $25 \mathrm{HD}$ patients, 25 presymptomatic HD (PreHD) subjects, 40 healthy controls. Six consecutive $\mathrm{T} 2 *$-weighted gradient echo-planar wholebrain volumes were acquired at different time of echo (TE) (TEs: 6, 12, 20, 30, 45 and $60 \mathrm{~ms}$; TR $=5000$; bandwidth $=1116$ $\mathrm{Hz} / \mathrm{vx}$; matrix size $128 \times 128 \times 80$; flip angle $90^{\circ}$; voxel size of $\left.1.5 \times 1.5 \times 2 \mathrm{~mm}^{3}\right)$. A mono-exponential signal decay curve was obtained and we calculated i.e. relaxation rates $\mathrm{R} 2 *=1 /$ T2*. A general linear model was used to test for differences between groups with sex and age included as covariates.

Results: An examination of $\mathrm{R} 2 *$ differences reveled a significant increase in the left CST in preHD subjects when compared to HD patients, $(\mathrm{F}(2,86)=3.46, p=013)$. HD patients did not have higher levels of iron in both CST, compared to healthy controls.

Conclusion: Our results indicate a change in WM microstructure between the presymptomatic phase and disease onset revealed by an increase of R2* (iron) in CST of PreHD subjects. An increase of iron content is associated with remyelination and with a higher density of oligodendrocytes, the brain cells with the highest iron, attempting to repair myelin damage. Our results suggest a higher number of oligodendrocytes, than normal in PreHD subjects that is in line with previous reports. Our results support the hypothesis that in HD, as in healthy older individuals, remyelination processes may successfully compensate during younger years (in PreHD stage), but eventually begin failing in older years (HD stage). Furthermore our result is consistent with the iron level asymmetry described in normal aging and movement disorders diseases; and confirming also the leftward-biased gray matter loss present in the striatum of HD patients.

\section{IS ALCOHOL \& TOBACCO ABUSE ASSOCIATED WITH EARLIER AGE OF ONSET \& FASTER DISEASE PROGRESSION, IN HUNTINGTON DISEASE?}

Remika Mito, Andrew Hayen, Jillian McMillan, Elizabeth McCusker, Clement Loy, The Huntington Disease Study Group COHORT Investigators

\begin{abstract}
.
Introduction and objectives: CAG repeat length is considered the most important determinant of age of onset for Huntington Disease (HD). However environmental factors may account for some of the remaining variability in age of onset, and rate of disease progression. We aimed to explore the effects of alcohol and tobacco abuse as possible environmental risk factors for HD.

Methods: We examined data collected in the COoperative Huntington Observational Research Trial (COHORT, 20062011). The relationship between alcohol or tobacco abuse, and age of HD onset, was examined using multiple linear regression. We used the log-transformed age of onset as our outcome variable, given the known non-linear relationship between CAG repeat length and age of onset. CAG repeat length and gender were included as covariates on an a priori basis, and alcohol or tobacco abuse as test variables in model building. For disease progression, we carried out a similar analysis, quantifying disease progression as the rate of change in Total Functional Capacity (TFC) score, between Baseline Visit and Visit One. Results: Alcohol abuse was found to be associated with an earlier age of HD onset $(N=954, p=0.0001)$, after adjusting for $\mathrm{CAG}$ repeat length and gender. In our model, a history of alcohol abuse brought forward the age of onset by 3.01 years, in a man with $42 \mathrm{CAG}$ repeats. No significant association was found between tobacco abuse and age of onset. Alcohol abuse was also found to be associated with a faster rate of decline in TFC $(N=792, p=0.046)$, after adjusting for $\mathrm{CAG}$ repeat length and gender. Conclusions: Amongst the individuals of this large observational study, alcohol abuse was associated with an earlier onset of HD, and faster disease progression. Avoidance of alcohol abuse may be beneficial for people carrying abnormal expansions in the HD gene.
\end{abstract}

\section{JUVENILE HUNTINGTON'S DISEASE IN A PERUVIAN COHORT}

Herbert Jose Capuñay Quiñones, Mario Reynaldo

Cornejo Olivas, Mario Renato Velit Salazar, Maria Victoria Marca Ysabel, Gerardo Olimpio Ortega Davila, Demetrio Saul Lindo Samanamud, Diego Martin Veliz Otani, Miguel Alfredo Martin Inca Martinez, Victor Land

Abstract.

Introduction: Infantile ( $\leq 10$ years at onset) and Juvenile (10 to 20 years at onset) forms of Huntington's Disease (HD), or 
simply juvenile HD (JHD), represent up to $8 \%$ of HD cases. JHD cases are mostly related to paternal transmission; typically presenting 60 or more CAG repeats in the HTT gene. Molecular diagnosis for HD is available in Peru since 2000, with a National Registry at Instituto Nacional de Ciencias Neurologicas (INCN).

Objective: To describe the clinical and molecular characteristics of juvenile and infantile forms of $\mathrm{HD}$ in this Peruvian cohort.

Methods: A retrospective, observational study was carried out by reviewing the HD registry at the Neurogenetics Research Center-INCN from 2000 to 2013; clinical records were also reviewed for each selected entry. CAG genotyping was performed by conventional PCR methods followed by of polyacrylamide gel electrophoresis using genomic DNA from white blood cells, CAG repeat counting was carried out by a lineal calibration curve.

Results: A total of sixteen juvenile HD cases from 16 families where identified out of 286 individuals with HD (5.6\%), 4 of them $(25 \%)$ corresponding to infantile forms. Mean age at onset for infantile and juvenile forms was $6 \pm 2.9$ [3,10 years] and $16.9 \pm 2.7$ [12, 20 years], respectively; CAG repeats mean was $66 \pm 15.1[44,97]$. Significative correlation between age at onset and mutant allele expansion was confirmed for this group, $r=-0.62, p=<0.01$. Ten JHD cases $(62.5 \%)$ were males. There was a $7 \pm 6.0$ years delay $[0,20]$ to JHD diagnosis. Paternal transmission was referred in $75 \%$ of cases. Language disorders (58\%), behaviour disturbances (50\%) and dysarthria (50\%) are the most frequent clinical manifestations in juvenile forms. Ataxia (100\%), bradykinesia (75\%) and seizures $(50 \%)$ are common in infantile forms.

Conclusions: Our review of 16 JHD affected individuals shows mostly severe and variable phenotype expression of the disease with dysarthria, ataxia, bradykinesia and behavioural disorders as the most frequent manifestations. JHD cases have larger CAG repeats and predominance of paternal transmission. There is a significant delay in diagnosis of JHD in these cases. This project was supported by Instituto Nacional de Ciencias Neurologicas, NIH Research Training Grant \#R25 TW009345 funded by the Fogarty International Center, the National Institute of Mental Health, the NIH Office of Director, the Office of Women's Health and the Office of AIDS Research.

\section{JUVENILE HUNTINGTON'S DISEASE, CLINICAL AND GENETICS CHARACTERISTICS OF PATIENTS FROM ARGENTINA}

Virginia Parisi, Jose Luis Etcheverry, Ana Sanguinetti, Emilia Gatto

\footnotetext{
Abstract.

Background and purposes: Huntington's disease (HD) is an autosomal dominant disorder characterized by motor, cognitive and psychiatric manifestations. The prevalence of Juvenile onset HD (JHD, onset $\leq 20$ years) ranges $1 \%$ to $15 \%$. JHD is classified as childhood ( $\leq 10$ years) and adolescent (11-20 years) onset; with parkinsonian-akinetic syndrome and dystonia as the dominant motor features. Additional neurological features include: cerebellar signs, epilepsy,
}

myoclonus, gait disorders and spasticity. Behavioural problems, cognitive decline are also common in JHD. The ethnicity background might play a role in modulating the phenotype.

Objective: to describe clinical and genetic characteristics of a series of JHD patients from a center in Argentina.

Patients and Methods: This observational study was approved by institutional review board. JHD patients were identified from our database. Inclusion criteria were: disease onset $\leq 20$ years and clinical diagnosis of HD with molecular confirmation or parent with clinical and molecular clinical diagnosis of HD. Demographic, epidemiological, clinical and genetic data were analyzed.

Results: 60 HD patients were identified. JHD was individualized in 6 women and 5 men (18\%), they had 40-80 nCAG repeats, and mean age at onset 12.9 years. Paternal inheritance was reported in 7/10 cases. Seizures occurred in 1 case, Westphal variant was identified in 3 patients and behavioral disorders were present in all cases. MRI showed caudate atrophy and putaminal hyperintensity on T2 in 4 patients. Childhood onset ( $\leq 10$ years) occurred in 3 patients (5\%), with a range of $43-80 \mathrm{nCAG}$ repeats.

Conclusion: Beside the small sample, the present study constitutes the first reported series of JHD in patients from Argentina. In these series JHD shows a higher prevalence and a less frequency of seizures than previous reported international series.

\section{LAQUINIMOD REVERSES THE ENHANCED IMMUNE RESPONSE IN MICROGLIA FROM HD MICE}

Blair R. Leavitt, Colúm Connolly, Pamela K Wagner, Liat Hayardeny, Michael R Hayden

\section{Abstract.}

Introduction: Huntington disease (HD) is an inherited neurodegenerative disease caused by a polyglutamine expansion in the huntingtin protein. The YAC128 mouse model of HD expresses a full-length human huntingtin transgene with $128 \mathrm{CAG}$ repeats and replicates key features of HD (including selective striatal neurodegeneration). Several previous imaging and neuropathological studies have implicated a role for microglia and neuroinflammation in HD pathogenesis. Mutant huntingtin expressing primary monocytes (human HD patients) and primary macrophages (YAC128) are overactive in response to lipopolysaccharide (LPS) stimulation. Laquinimod is an experimental immunomodulatory drug which has been shown to modify astrocytic and microglial activation via an NFkB-mediated mechanism of action.

Hypothesis: Mutant huntingtin expression in microglia and astrocytes leads to altered inflammatory function of these cells influencing the pathogenesis of HD; Laquinimod treatment will reverse these neuroinflammatory cellular changes.

Objective: Our overall goal for this study was to understand the effect of Laquinimod on microglia in an ex vivo cellular model of Huntington disease.

Methods: We have recently developed techniques to isolate and culture primary microglia from YAC128 mice, and now 
use this system as an ex vivo model of HD neuroinflammation to evaluate possible therapeutic approaches for this devastating neurodegenerative disorder. Primary microglia from YAC128 mice differentially express a wide number of cytokines compared to wildtype microglia cultures in response to inflammatory stimulation. Results: This study examined the effect of Laquinimod on the enhanced immune response of microglia expressing mutant huntingtin protein. Cultured primary microglia from YAC128 mice secrete elevated levels of inflammatory cytokines compared to wildtype microglia in response to LPS and matrix metalloproteinase 3 stimulation, and this hypersecretion phenotype can be ameliorated by treatment with Laquinimod. Laquinimod treatment decreased p65 nuclear localization, NFkB-mediated transcriptional activity, and activation in YAC128 microglia.

Conclusion: Our data demonstrate that Laquinimod can reverse the neuroinflammatory phenotype of YAC128 microglia, is a promising drug treatment for $\mathrm{HD}$, and suggest that primary microglia cultures can be used to screen for potential therapeutics that may modulate neuroinflammation in HD.

\section{LATE ONSET HUNTINGTON'S DISEASE IN A PERUVIAN COHORT}

Victor Landsteiner Gomez Calero, Mario Renato Velit Salazar, Mario Reynaldo Cornejo Olivas, Gerardo Olimpio Ortega Davila, Maria Victoria Marca Ysabel, Demetrio Saul Lindo Samanamud, Diego Martin Veliz Otani, Miguel Alfredo Martin Inca Martinez, Herbert J

\section{Abstract.}

Introduction: Late onset cases of Huntington's disease (HD) have an age at onset of 60 years or greater, accounting for $10-25 \%$ of HD cases worldwide. Important characteristics include a lower number of CAG repeats in the HTT gene, frequent absence of family history, mild motor dysfunction with a slow progression, and mild cognitive impairment. Molecular diagnosis for HD has been available in Peru since 2000 at Instituto Nacional de Ciencias Neurologicas (INCN), where the National HD Registry has been developed.

Objective: To describe the clinical and molecular characteristics of late onset HD in a Peruvian cohort.

Methods: A retrospective, observational study was carried out by reviewing the HD registry at the Neurogenetics Research Center-INCN from 2000 to 2013; clinical records were also reviewed for each selected entry. CAG genotyping was performed by conventional PCR methods followed by of polyacrylamide gel electrophoresis using genomic DNA from white blood cells, CAG repeat counting was carried out by a lineal calibration curve.

Results: Twenty-six late onset HD cases from 21 families were identified from 286 individuals with HD (9.1\%). Mean age at onset was $64.5 \pm 4.4$ [61, 76 years] and CAG repeats mean was $41.8 \pm 1.5[40,46], r=-0.38, p=0.054$. Twelve individuals (46.2\%) were male; eight cases (30.8\%) originated from Cañete valley (region with high prevalence of HD) and five (19.2\%) from Lima (capital city). Eighteen individuals $(69.2 \%)$ had a positive family history, 9 of them $(34.6 \%)$ with paternal transmission. Frequent clinical manifestations include mild chorea $(69.2 \%)$, dysarthria
(53.8\%) and dystonia (30.8\%). Mood disturbances were observed in 11 individuals $(40.7 \%)$. UHDRS III motor score ranged $22.7 \pm 8.6[4,43]$. MMSE and MoCA average scores were 21.9 and 14.6, respectively.

Conclusions: Our review of 26 late onset HD affected individuals shows a mild phenotype expression of the disease, associated with low range of CAG repeats and up to $30 \%$ of cases with absence of clear family history. Cañete valley remains the region with more cases in this late onset $\mathrm{HD}$ cohort. This project was supported by Instituto Nacional de Ciencias Neurologicas, NIH Research Training Grant \#R25 TW009345 funded by the Fogarty International Center, the National Institute of Mental Health, the NIH Office of Director, the Office of Women's Health and the Office of AIDS Research.

\section{MITOCHONDRIAL DNA COPY NUMBER IN PERIPHERAL LEUCOCYTES FROM HUNTINGTON'S DISEASE MUTATION CARRIERS DISPLAYS A BIPHASIC PATTERN OF PRE-ONSET INCREASE AND POST-ONSET DECLINE}

Maria Petersen, Esben Budtz-Jørgensen, Sven Asger Sørensen, Jørgen Erik Nielsen, Signe Marie Borch Nielsen, Anne Nørremølle

\section{Abstract.}

Introduction: Mitochondrial dysfunction has been proposed to play a central role in the pathogenesis of Huntington's disease (HD). The mitochondrial DNA (mtDNA) encodes key factors in mitochondrial processes, and reduced mtDNA copy number per cell has been found associated with neurodegeneration in mtDNA depletion syndromes. We hypothesize a reduction in mtDNA copy number in HD could based on observed increased frequency of mtDNA deletions and reduced activity of peroxisome proliferators-activated receptor gamma coactivator 1 alpha (PGC-1 $\alpha)$ and mitochondrial transcription factor A (TFAM), both known to stimulate mtDNA replication. In HD, previous investigations of mtDNA copy number in peripheral leucocytes have shown conflicting results and further studies are needed.

Objectives: To study in detail the copy number of mtDNA relative to nuclear DNA in peripheral leucocytes from HD mutation carriers, and to correlate this to age and duration of disease.

Methods: DNA was isolated from blood samples using standard methods. mtDNA and nuclear DNA copy numbers were assessed using Q-PCR. Estimation of age at onset was based on the appearance of motor symptoms.

Results: Comparison of mtDNA copy number per nuclear DNA in leucocytes from 41 HD mutation carriers and 28 agematched controls showed a significant lower mtDNA copy number in the HD carriers, and an inverse correlation between their age and mtDNA copy number. Analysis of a different cohort of HD patients $(N=53)$ confirmed a lower average mtDNA copy number but failed to show any correlation to disease duration. We noted a large variation between individuals and in order to follow the mtDNA copy number changes in individual patients we searched our DNA archive; 
for 26 of the patients we found preceding DNA samples, enabling us to analyze and compare two consecutive samples from the same patient. Analysis of these sample pairs revealed a biphasic pattern of mtDNA copy number with pre-onset increase and post-onset decline.

Conclusion: The mathematical model describing our results from $26 \mathrm{HD}$ patient sample pairs indicates that the mtDNA copy number is increasing in the years up to disease onset, which may be explained by compensatory mechanisms trying to maintain the necessary cellular level of mtDNA. However, the post-onset decline suggests that these compensatory mechanisms at some point fail to keep up. Whether this is a cause of HD pathogenesis or a consequence, remain to be investigated.

\section{MORPHOLOGICAL AND FUNCTIONAL CHANGES IN MOTOR ENDPLATES IN A MURINE MODEL OF HUNTINGTON'S DISEASE}

Bárbara Campos de Aragão, Hermann Alecssandro Rodrigues, Toniana Gonçalves de Carvalho, Fabíola Mara Ribeiro, Cristina Guatimosim

Abstract. The Huntington's disease (HD) is a neurodegenerative disorder characterized by a progressive decline of motor and cognitive functions including involuntary movements of the limbs and face, personality changes and dementia. It is caused by a CAG trinucleotide repetition that leads to a polyglutamine expansion in the huntingtin protein. It is known that huntingtin is highly expressed in the central and peripheral nervous systems. Within neurons, this protein binds to synaptic vesicles and interacts with several proteins associated with vesicular transport. Thus, it is suggested that the mutant huntingtin may interfere with the release of neurotransmitters causing synaptic dysfunction, which may worsen to motor impairment characteristic of the disease. Previous data indicated a reduction in cholinergic neurotransmission, as well as motor impairment in animal models of HD. Based on this evidences, this study aims to investigate alterations in the neuromuscular junction (NMJ) and in muscle strength in BACHD mice, a transgenic model for HD. This model represents a new and robust in vivo paradigm for studying the pathogenesis of HD. Briefly, for evaluation of muscle strength, BACHD and WT mice were subjected to wire hanging test. BACHD mice showed significant differences in muscle strength compared with WT. For optical analysis, neuromuscular preparations were isolated and stained with the dye FM1-43fx and $\alpha$-bungarotoxin-Alexa 594 to visualize presynaptic terminals and acetylcholine receptors clusters, respectively. Using confocal microscopy, we analyzed morphological parameters in BACHD and WT animals. We observed that pre and postsynaptic elements presented less fluorescence in BACHD which may reflect denervation. We looked at presynaptic nerve terminals previously labeled with the exoendocytic marker FM1-43 and we noticed that synaptic vesicle exocytosis was impaired in BACHD. Moreover, ultrastructural analysis revealed that even though the synaptic elements morphology in BACHD and WT are similar, the synaptic vesicles in BACHD synaptic terminals have a different shape compared to WT. Our results so far suggest a peripheral functional impairment in NMJs of BACHD mice that might contribute to the motor defects seen in this mouse model of Huntington's disease. Supported by CNPq, CAPES and FAPEMIG.

\section{MRI BASED FAT IMAGING STRATEGIES FOR QUANTIFICATION OF BROWN AND WHITE ADIPOSE TISSUE IN HD MOUSE MODELS USING A SMALL ANIMAL MRI SCANNER}

Axel Bornstedt, Patrick Weydt, Hans-Peter Müller, Jan Kassubek, Volker Rasche, Katrin S. Lindenberg

Abstract. Many Huntington's Disease (HD) patients show an unintended weight loss and skeletal muscle wasting with normal or even high food intake already at early stages of the disease. Metabolic abnormalities are described for peripheral tissues in HD patients and HD mouse models, especially for the highly metabolic tissues like white adipose tissue (WAT) and skeletal muscle. In addition alterations in brown adipose tissue (BAT) including failure in thermogenesis are found in HD mouse models. Multi-point chemical-shift magnetic resonance imaging (MRI) methods enable the direct separation of fat and water bound protons relying on the unique chemical shift of either tissue. Due to the metabolic activity of BAT, the amounts of water-bound protons differ between WAT and BAT. The aim of the study was to investigate the potential of fat-water fraction imaging for the identification and quantification of WAT and BAT in WT and HD mouse models. Five wildtype and 5 HdhQ111 knock in mice were included. All MRI data was acquired at $11.7 \mathrm{~T}$ (BioSpec 117/16, Bruker) using a one-element body resonator for signal reception. Fat/ water fraction imaging was performed after sacrifying the animals by a fast three-point imaging technique as proposed earlier by Reeder et al. [1]. Images were acquired with a FLASH sequence at - $/ 6, \quad / 2,7 \quad / 6$ phase difference at echo times of $1.118 \mathrm{~ms}, 1.313 \mathrm{~ms}$, and $1.507 \mathrm{~ms}$ respectively. Spatial resolution was as $120 \times 200 \mu \mathrm{m}^{2}$. Overall acquisition time resulted as $3 \min 50 \mathrm{~s}$ for the complete investigation. Complete coverage of the neck region was achieved by acquiring 9 slices of $500 \mu \mathrm{m}$ slice thickness and $1 \mathrm{~mm}$ spacing. Fat water fractions were calculated and BAT was identified as fat tissue showing $50-80 \%$ fat, whereas WAT was identified by fat water fractions showing more than $80 \%$ fat. The BAT and WAT could be clearly separated by eyeballing in the images showing almost opposed phase for water and fat protons (7 /6). Fat water fractions could be reconstructed and the BAT could be clearly delineated from the WAT in the fat water fraction data. Clear differences in the BAT volume between the two different cohorts were observed, with a clear reduction of BAT in the transgenic group. It can be concluded that fat water fraction imaging can be applied for identifica- 
tion and quantification of BAT in a HD knock in mouse model. Since all data was acquired applying a conventional FLASH imaging technique, the results should be translatable to human applications.

\section{MUTANT HUNTINGTIN FRAGMENTATION IN IMMUNE CELLS TRACKS HUNTINGTON'S DISEASE PROGRESSION}

\author{
Dr Edward Wild, Dr Ulrike Träger, Dr Davina \\ Hensman, Nicola Robertson, Dr Andreas Weiss, \\ Stephan Grueninger, Ruth Farmer, Dr Christian \\ Landles, Dr Rachael Scahill, Dr Nayana Lahiri, \\ Dr Salman Haider, Dr Douglas Macdonald, \\ Michela Tessari, Dr David F Fisch
}

\begin{abstract}
Quantification of mutant huntingtin in accessible human tissues is desirable but challenging. We used a timeresolved Förster resonance energy transfer (TR-FRET) immunoassay to quantify mutant and total HTT levels in HD patient leukocytes. We used a novel electrochemoluminescence (ECL) assay to quantify mHTT in unsorted leukocyte samples. Mutant HTT levels in peripheral immune cells differed significantly between HD patients and controls, and between pre-manifest and manifest disease. Monocyte and T-cell mutant HTT levels were significantly associated with disease burden scores and caudate atrophy rates in HD patients: the first instance where quantifying a pathogenic agent in blood cells predicts structural brain change in a neurodegenerative disease. Using immunoprecipitation, we show that full-length mutant huntingtin is cleaved to N-terminal fragments in immune cells. The novel electrochemoluminescnce assay is sufficiently sensitive and accurate to quantify and reproduce our findings in unsorted leukocyte samples fractionated from $8 \mathrm{ml}$ whole blood, a methodology more suited to multisite clinical trials. Ex-vivo HTT quantification in peripheral cells may be useful for clinical trial stratification. Quantifying the progressive increase of mutant HTT levels in peripheral leukocytes may provide one valuable means, of monitoring disease progression in Huntington's disease.
\end{abstract}

\section{NEUROPSYCHIATRIC SYMPTOMS DURING PRE-MOTOR STAGE IN HUNTINGTON'S DISEASE}

Saul Martinez-Horta, Jesus Perez Perez, Mar Carceller Sindreu, Ramon Fernandez de Bobadilla, Javier Pagonabarraga, Antonia Campolongo, Berta Pascual Sedano, Jaime Kulisevsky

\footnotetext{
Abstract.

Introduction: Huntington's disease (HD) is characterized by a progressive development of motor, cognitive and neuropsychiatric symptoms. While clinical diagnosis is based on the unequivocal presence of motor symptoms, neuropsychiatric manifestations can precede by years the motor disease onset. Objectives: To study the presence and characteristics of neuropsychiatric symptoms during the pre-motor stage in HD as compared to a matched sample of healthy controls.
}

Methods: Forty-seven participants with genetic confirmation $(\mathrm{CAG} \geq 36)$ for HD disease in the pre-motor stage (UHDRS $\leq$ 4; TFC $\geq 12$ ) and nineteen healthy controls. Neuropsychiatric symptoms (depression, irritability, apathy, psychosis and executive dysfunction) were assessed using the short form of the Problems Behavior Assessment Scale (PBA-S) and establishing a score $\geq 2$ as clinically meaningful.

Results: No differences were found between groups for clinical and demographic variables (age $=35.9 \pm 13.1$ vs 38.3 $\pm 13.1 ; t, p=0.436 ;$ UHDRS $=1.1 \pm 1.3$ vs $1.8 \pm 2.9 ; t$, $p=0.333 ; \quad \mathrm{TFC}=12.3 \pm 2.7 \quad$ vs $12.9 \pm 0.7 ; \quad t, \quad p=0.509)$. Significance differences were found for apathy $(2.13 \pm 4.3 \mathrm{vs}$ $0.5 \pm 1.3 ; t, p=0.03)$ and psychosis $(0.4 \pm 1.3$ vs $0.0 ; t$, $p=0.02$ ) scores. Up to $26 \%$ of the pre-motor participants scores $\geq 2$ on apathy vs $15 \%$ of healthy controls. Apathy $(r=-0.379 * *)$ and psychosis $\left(r=-0.319^{* *}\right)$ correlated significantly with the functional status of the patients (TFC). Conclusion: More sever apathetic symptoms constitutes a clinical characterized that should be recognized from the premotor stage of HD that is related to the functional status of the patient. Despite is not clinically significant some degree of psychotic symptoms also related to functional status, differentiates patients to healthy controls.

\section{NEUROPSYCHIATRIC SYMPTOMS IN HUNTINGTON'S DISEASE IN A EUROPEAN COHORT (REGISTRY)}

Erik van Duijn, David Craufurd, Anna A.M. Hubers, Erik J. Giltay, Raphael Bonelli, Hugh Rickards, Jenny Callaghan, on behalf of the EHDN Behavioural Phenotype Working Group

\section{Abstract.}

Introduction: The majority of Huntington's disease (HD) mutation carriers experience some psychopathology during their lifetime, varying from a bad temper to psychosis. Prevalences of neuropsychiatric symptoms vary widely due to diverse study populations in different stages of HD and the use of different assessment methods with varying definitions of psychiatric phenomena.

Methods: The study population consisted of 1,993 HD mutation carriers from 15 European countries, all participating in the observational REGISTRY study of the European Huntington's Disease Network. The behavioral section of the Unified Huntington's Disease Rating Scale was used to examine the prevalence and correlates of five neuropsychiatric features: depression, irritability/aggression, obsessive/ compulsive behaviors, apathy, and psychosis.

Results: Of all participants $12.7 \%$ were moderately to severely depressed, $13.9 \%$ had moderate to severe irritable/aggressive symptoms, $13.2 \%$ showed moderate to severe obsessive/ compulsive behaviors, $28.1 \%$ were moderately to severely apathetic, and $1.2 \%$ had moderate to severe psychotic symptoms. Only $54.9 \%$ of all participants with moderate to severe depression used antidepressants, suggesting undertreatment of depression. A history of depression was correlated with all neuropsychiatric symptoms. Apathy had a strong inverse correlation with TFC score. The low prevalence of psychosis may be explained by the rather young population (mean age 50.3 years) and the relatively high use of antipsychotics. 
Conclusion: In this large European observational study, the high prevalence of a variety of neuropsychiatric symptoms in Huntington's disease was confirmed. Our next step will be analysis of longitudinal data and we recommend studies on the treatment of these neuropsychiatric symptoms.

\section{NEW INDUCIBLE DROSOPHILA MODELS OF HUNTINGTON'S DISEASE}

\section{Raheleh Heidari, Hèrve Tricoire}

\begin{abstract}
.
Introduction: Huntington's disease (HD) is a devastating progressive neurodegenerative disease primarily caused by CAG repeat expansions in the exon 1 of huntingtin gene. The CAG repeat encodes polyglutamine tract in huntingtin protein. The length of CAG repeats mediates the age of onset and the severity of disease and HD patients suffer from a range of cognitive, psychiatric and motor impairments. Although HD is best known as a neurodegenerative disorder, cardiac failure is the second cause of death in HD patients. Despite vigorous investigations on pathology of $\mathrm{HD}$, the direct role of mutant huntingtin on pathology of cardiovascular disease associated with HD and consequently the therapeutics strategies remain unknown.

Objectives: We aim to establish Drosophila models, with adult specific expression of disease; our objective is to spatially control the expression of mutant huntingtin in different tissues and organs including neurons, glia, heart and eyes, by investigating the phenotypic expression of disease we evaluate physiological and behavioral outputs of the experimental models and consequently implementing them into screening the therapeutic compounds for HD.

Methods: By application of gene switch system (GS) we have been able to regulate the tissue specific expression of mutant huntingtin in neurons, glia, heart and eyes. Using behavioral assays including locomotor activity and feeding behavior we measured the circadian rhythm outputs of the models and in addition, using in vivo imaging, we recorded the cardiac cycle of HD flies.

Conclusions: We report that adult specific neuronal and glial expressions of mutant huntingtin drastically shorten the life span. Expression of mutant protein in PDF neurons results in disturbed circadian rhythmicity and perturbed locomotor activity. We also demonstrate that expression of mutant htt in myocytes of adult heart drastically alters the systolic and diastolic features of cardiac cycle.
\end{abstract}

\section{NEW PERSPECTIVE OF CARE IN HUNTINGTON'S DISEASE}

\section{Helena Soares}

Abstract. The Huntington's disease doesn't affect only de patient but it also affects considerably the life of the patient's family. Generally, these assume an informal care of the patient. The informal care takes to a physical and emotional effort described in the scientific literature as a caregiver overcharge, beyond the difficulties in several areas of their life like alterations in their family and social life, economic and work problems, (Brito 2000; Cook et al., 1996; Fadden et al., 1987; Goodman et al., 1997). This research resulted from the consideration of the caregiver's situation as a health problem in the way that it doesn't affect only themselves but also the patients target of care (Pinto et al., 2005).Therefore, the purpose of this research was finding the overcharge and the difficulties of the Huntington's patient caregiver and in what way can these difficulties be related with the social and demographic characteristics and with the strategies used to overcome them. For that, a quantitative study described as transversal has been made where have been included 40 Huntington's patient caregivers registered in the Portuguese Association of Huntington's Disease Patients. We observed in our study that are predominant the female caregivers aged over 50 years, married, mostly retired, providing care 24 hours a day, with family relationship and cohabiting with the patient. We noticed then that the caregivers show a overcharge mostly psychological $(57.5 \%)$ and social however, $55 \%$ of the caregivers don't show economic difficulties. We verified that the main difficulties shown by the caregivers are physical exhaustion, $67.5 \%$ show difficulties to sleep, decrease of the life quality and lack of support by the health and social services. Besides the difficulties, $52.4 \%$ still consider themselves capable of continue providing care of their familiar, and $77.5 \%$ feel closer by giving care. With this research it was possible to establish the relationship between the variables under study in order to be defined an intervention proposal that meets these difficulties.

\section{NEW PERSPECTIVES IN ENERGY METABOLISM IN NORMAL AND HD HUMAN BRAIN: LOCALISATION OF CREATINE TRANSPORTER AND CREATINE KINASE ISOFORMS IS DEPENDENT ON CELL TYPE AND FUNCTION}

Henry Waldvogel, Mathew Lowe, Eric Kim, Richard Faull, David Christie

\section{Abstract.}

Introduction: Creatine supports cellular energy metabolism in many high-energy tissues including skeletal muscle and the brain. For this reason, it is currently being tested in clinical trials as a neuroprotective agent in Huntington's disease (HD) and Parkinson's disease (PD). Surprisingly little is known, however, about the distribution of the proteins that control creatine functioning in the brain.

Objective: This study characterises the distribution of the proteins which control creatine function in the human brain and measures changes that occur in these proteins in the HD motor cortex. Methods. Immunohistochemical staining was adopted with both light and confocal laser scanning microscopy to determine the distribution of the two creatine kinase (CK) isozymes and the creatine transporter $(\mathrm{CrT})$. Additionally, qPCR and Western blotting was used to measure changes in the HD brain.

Results: In the normal brain, the cytosolic isoform of CK (BCK) was found predominantly expressed in astrocytes with only selective expression in neurons. The mitochondrial CK isoform (uMtCK) was selectively expressed in neurons- and 
demonstrated that BCK and uMtCK are rarely expressed strongly together in the same cell [the exceptions being parvalbumin containing neurons, medium spiny striatal neurons (MSNs) and variable localisation in cortical neurons], while CrT showed variable expression in different neuron types. In the human HD cortex we demonstrate that there are lower protein levels of BCK and also marked loss of both CrT-expressing and uMtCK-expressing neurons, in line with previous studies showing lower BCK levels in the neurons in the human striatum.

Conclusions: These data indicate that cells express the creatine system proteins in a heterogeneous manner that matches their energetic requirements, so that promoting mitochondrial ATP production is mainly through uMtCK in neurons and providing an energy buffer/shuttle is through BCK mainly in astrocytes. In HD motor cortex the loss of uMtCK and BCK from the HD brain suggests that there may be a disruption in the creatine circuit within brain cells, and this may contribute to the energetic deficits seen in HD. From these morphological studies the therapeutic supplementation of creatine in HD may be beneficial to cortical neurons which have the CrT and therefore indirectly benefit MSNs, but may not be directly beneficial to $\mathrm{CrT}$ deficient medium spiny neurons.

\section{OCULOMOTOR AND EXECUTIVE FUNCTION IN HUNTINGTON'S DISEASE: AN FMRI STUDY}

Gina Caetano, Filipa Júlio, Cristina Januário, Miguel Castelo-Branco

\footnotetext{
Abstract.

Background: Huntington Disease (HD) morphometric neuroimaging studies have shown that neural degeneration starts one to two decades prior to symptoms onset, with an emphasis on striatal neuronal loss. In contrast, functional magnetic resonance imaging (fMRI) underpins brain activity, and thus may detect changes related to dysfunction prior to neuronal death. FMRI studies, independently of the experimental paradigm, most often report cortical hyperactivation in gene carriers further from estimated symptom onset, whereas hypoactivations are usually reported for groups close to estimated onset. Here, we aim at studying oculomotor function and the effect of executive and/or memory load. We expect to unravel brain activation patterns related to dysfunction within the Basal ganglia-thalamocortical circuits.

Methods: Functional brain images were acquired during prosaccades (PS), antisaccades (AS), and n-back memory antisaccades (MAS), in separate runs, using an fMRI blockdesign. Eye movements were measured during scanning. The pool of participants include a premanifest HD (preHD) group $(N=10)$, a Stage1 HD group $(n=7)$, and a healthy age- and education-matched control group $(N=13)$. A comprehensive battery of neuropsychological tests was used to assess the overall cognitive functioning of the participants.

Results: The neuropsychological evaluation showed significant differences between Stage $1 \mathrm{HD}$ and preHD/control groups (Mann-Whitney, $p<0.05$ ). For each of the groups, the saccadic paradigms activated a network of regions commonly
}

reported in oculomotor studies. Contrast maps between Controls and preHD groups (RFX, cluster thresholding:20) unveiled decreased recruitment of dACC/pre-SMA, caudate, DLPC, and middle occipital gyrus in preHD for the AS task. Similarly, dACC/preSMA, ventral striatum, DLPC, middle occipital gyrus, PEF, FEF and IFG show decreased recruitment in preHD for the MAS task. Comparatively to preHD, Stage 1 HD participants show an increased recruitment of several areas (including visual cortex, precuneus, insula, between other) for the same tasks.

Remarks: Although controls and preHD groups showed no overt cognitive differences, these preliminary results have unveiled differential recruitment of cortical and basal-ganglia regions for saccadic tasks involving inhibitory processes with/without executive load. In overall, these results are in accordance with known disease neuropathology.

\section{OFFSPRING OF A PARENT WITH HUNTINGTON'S DISEASE: CHILDHOOD EXPERIENCES AND ADULT PSYCHOLOGICAL CHARACTERISTICS}

Lucienne van der Meer, Erik van Duijn, Aad Tibben

Abstract.

Introduction: Persons at risk for Huntington's Disease (HD) may have been exposed to the disease process of their affected parent during childhood. Growing up with a parent affected with HD may negatively affect offspring's development and well-being in later life.

Objective: To investigate childhood experiences and psychological characteristics in adult offspring of a parent with HD. Methods Self-report scales were used to assess adverse childhood experiences, adult attachment style (attachment anxiety and attachment avoidance), and mental health, in adult offspring of a parent with Huntington's Disease (HD). This group was compared to a reference group of persons who did not have a parent with HD. Associations between childhood experiences and adult psychological characteristics were investigated.

Results: Offspring of a parent with $\mathrm{HD}(n=74)$ reported more parental dysfunction and parental psychiatric problems in childhood, compared to the reference group $(n=127)$. Parental loss was also relatively common in HD-offspring. HDoffspring reported a significantly higher number of adverse childhood experiences than the reference group. Compared to the reference group, HD-offspring showed more adult attachment anxiety and poorer mental health. No significant associations were found between childhood experiences and adult attachment style or mental health.

Conclusions: This cross-sectional study indicates that, in childhood, offspring of a parent with HD may be exposed to dysfunction and psychiatric problems of their parent. Adult offspring of a parent with HD may have a higher level of attachment anxiety and poorer mental health than persons without an HD background. Longitudinal data and in-depth exploration of family dynamics, parent-child interactions and parenting styles are needed to enhance understanding of relationships between childhood experiences and adult attachment styles in offspring of a parent affected with HD. 


\section{PATIENT EXPERIENCE OF GENETIC DIAGNOSIS OF HUNTINGTON'S DISEASE - A RETROSPECTIVE SURVEY}

\author{
Richard Roxburgh, David Bourke, Jo Dysart, \\ Miriam Rodrigues
}

\begin{abstract}
.
Introduction: The delivery of a genetic diagnosis has the potential to be a very traumatic experience, especially when the disease is incurable and likely to cause significant morbidity such as for Huntington's disease (HD) and myotonic dystrophy (DM). Patients' experience of this process has been studied in small qualitative studies but systematic surveys have not been undertaken previously.

Objective: The purpose of the study was to survey the whole experience of genetic testing: the patients' preparation for having the genetic test; the time of receiving genetic test results and the follow up after testing.

Method: We constructed an anonymous postal questionnaire with 36 multichoice answers. It was sent to all patients in the neurogenetics clinic who were considered capable of answering the questionnaire. Responses to each of the questions were rated as being satisfactory or unsatisfactory. The results were analysed on a patient-by-patient basis and a question-by-question by question basis. Factors that might influence the outcome such as recency of diagnosis, clinician involved, diagnostic vs predictive testing were examined.

Results: 70 patients with HD and 69 with DM were mailed the questionnaire. In the HD group $21(30 \%)$ completed questionnaires were received. 17 of the 21 patients appeared almost entirely happy with their experience with 6 or fewer "unsatisfactory" answers compared with 12-19 "unsatisfactory" answers in the other 4 . Best results were seen when the HD specialist nurse was involved; the worst results were seen in a patient who received results from someone with no training in genetics. There were no differences between the last five years and previous years; or between predictive and diagnostic testing. Specific questions that gave unsatisfactory answers were that patients were not given the option of whom to receive their test results from, perceived inadequate support for family members at time of diagnosis and of patients after diagnosis. However, results for the HD group were considerably better than for the DM1 patients.

Conclusion: Most patients have a good experience of their diagnosis, attention to the outliers in this study reveals areas that we can work on. The better experience of patients going through genetic testing for HD compared with DM is probably due to our having an explicitly dedicated Huntington's disease service and to clinicians taking extra care with these patients.
\end{abstract}

\section{PHYSICAL ACTIVITY AS AN ENVIRONMENTAL MODULATOR OF GENETIC DETERMINISM IN HD: THE FIT-HD STUDY}

Anita M Goh, Anthony Hannan, Kay Cox, Nicola Lautenschlager

Abstract. HD has long been viewed as the quintessential example of genetic determinism. However, research has demonstrated that environmental factors play a significant role in modulating the disease process, including the age of onset. In transgenic mouse models of HD, co-author Hannan's laboratory has demonstrated that environmental enrichment (enhanced motor, cognitive and sensory stimulation) delays onset and progression of motor symptoms and neural degeneration. Introducing an exercise wheel to young HD mice delays onset and progression of motor symptoms, and reverses reduction of brain derived neurotrophic factor in the striatum and hippocampus. Such stimulation has also ameliorated deficits in learning and memory, as well as depressive behaviours in HD mice. Physical activity (PA) has been found to have beneficial neural effects in older adults, with increases in hippocampal volume and cognition, apparent even when exercise is limited to later life. We have found that PA improved cognitive function in those at risk of Alzheimer's disease (AD), and are conducting randomized controlled trials (RCT) investigating this PA program in AD patients, older adults at risk of $\mathrm{AD}$ with vascular risk factors or sedentary lifestyle, and in older care recipients and carers. The role of exercise as a potential modifier is receiving increasing attention in HD. Avoiding passivity was found to be a potential beneficial modulator in $\mathrm{HD}$, and a PA program improved in motor function in HD patients. However, evidence from RCTs is lacking. AIMS of this study are to: 1) examine the feasibility of our PA program in FIT-HD (by measuring adherence, acceptability, benefits, risks); 2) improve physical and mental health and quality of life in HD carriers. HYPOTHESES. Participants randomised to the PA program will: 1) increase PA and adhere to the protocol; have 2) improved quality of life and fitness, 3) improved sleep, 4) less motor and cognitive decline, 5) improved resting brain state, and 6) have less depressive symptoms, as compared to participants randomised to usual care.

Methods: RCT of a validated 24-week PA intervention in HD in Australia. Participants are randomly allocated to an education/usual care group or to a 24-week home-based PA program.

Results: PA should be more promoted in the HD community due to multiple health benefits. Should our RCT be completed successfully and our hypotheses supported then this could change clinical care of HD.

\section{PHYSICALTHERAPY - GAIT AND BALANCE IN HUNTINGTON DISEASE: A SYSTEMATIC REVIEW}

\author{
Tamine Capato, Monica Haddad, Maria Elisa \\ Piemonte, Egberto Reis Barbosa
}

\begin{abstract}
.
Background: Huntington's disease (HD) is a disorder characterized by involuntary movement characterized by chorea. However, what is the cause functional impairment that slows gait bradykinesia, postural adjustment and movement functional generating increased risk of fall and loss of functional independence. Cognitive deficits are associated with degeneration of the striatum and disruption to the frontal-subcortical neural circuits. Disorders of executive function and memory reflected by difficulty in maintaining attention to a task, planning, and initiating activity. There are littlies evidence that physiotherapy improve balance and gait.
\end{abstract}


Objective: Was indentify the most use trainings to improve balance and gait for improve the quality of life in HD.

Methods: The literature was retrieved via a systematic search using a combination of key words that included HD, physiotherapy, balance and gait. The electronic databases for Medline, Embase, CINAHL, Cochrane Controlled Trials Register, LILACs and SciELO, were searched up to May 2008. Articles meeting the review criteria were graded for study type and rated for quality using checklists to assess study validity and methodology. The classification ranks study evidence as systematic review; observational, randomized and nonrandomized intervention studies; no experimental studies; and expert opinion.

Results: A few studies proposed physiotherapy treatment for HD and these articles showed interventions varied as flexibility training, balance and gait. In 2008 there was still no consensus about the association of physiotherapy treatment with medication for DH. Since then, studies have emerged that suggest cognitive and attention strategies that can assist in the treatment of DH and improve gait and activities of daily living. Those demonstrate vehemently recommendations that physical therapy in conjunction with medication is effective for $\mathrm{HD}$, and suggested that is the key to treatment of HD to improve gait, balance and facilitation of functional mobility. Conclusion: The gait training, balance and functional mobility apparently can assist patients in early stages of DH maintain independence for activities of daily living and minimize secondary complications caused by the $\mathrm{DH}$. However, further clinical studies are needed that can support and improve care physiotherapy associated with medication in clinical practice, creating a guide of recommendations for care for DH as well as electing the best exercise protocols.

\section{POLISH CONTRIBUTION TO INTERNATIONAL STUDIES OF RARE DISEASES, AN EXAMPLE OF HUNTINGTON'S DISEASE}

\section{Daniel Zielonka, Jerzy T. Marcinkowski}

\begin{abstract}
.
Introduction: Huntington's disease (HD) is a genetic, progressive neurodegenerative disorder. Being rare it is difficult to collect a large enough cohort for significant interventional and environmental studies.

Objectives: The aim of this study was to assess rare diseases studies development in post-communistic country, never before involved in such studies, on an example of Huntington's disease.

Methods: International cohort HD research initiatives were reviewed in the U.S. National Library of Medicine National Institutes of Health (PubMed), Clinical Trials - a service of the U.S. National Institutes of Health (www.clinicaltrials. gov) and an archive of the HD research Coordination Centre for Poland located at the Chair of Social Medicine of Poznan University of Medical Sciences, Poland. Results Fifehundred-eighty-three participants were investigated in two observational and three clinical studies. The core study for such research initiatives is REGISTRY; a prospective, observational, longitudinal study on the rate of HD progression collecting large number of data on patients during annual
\end{abstract}

visits. HD studies participants number as well as number of cross-sectional and clinical studies increased dramatically in last years. Based on REGISTRY and other studies data base patients are easily and quickly collected for any other studies. Conclusions: Due to its large population Poland has become a target country for such research. REGISTRY, a novel approach, to overcome difficulties in patient collection for Huntington's disease interventional and environmental studies, effectively increase number of enrolled studies participants.

\section{POPULATION PHARMACOKINETIC ANALYSIS OF PRIDOPIDINE IN HEALTHY VOLUNTEERS AND PATIENTS WITH HUNTINGTON'S DISEASE}

Laura Rabinovich-Guilatt, Henri Merdjan, Rene Bruno, Esther Lukasiewicz-Hagai, Ofer Spiegelstein, Merav Bassan, Mathilde Marchand

\section{Abstract.}

Background: Pridopidine belongs to a new class of pharmaceutical agents, the dopidines, which have dopaminergic stabilizing properties and is being developed for the treatment of Huntington's disease (HD). Pridopidine is eliminated partly by urinary excretion and partly by hepatic metabolism, primarily via CYP2D6, to form one main inactive metabolite TV-45065. Time-dependent pharmacokinetics was observed in extensive metabolizers due time-dependent auto-inhibition of CYP2D6.

Objective: To perform a combined population pharmacokinetic (PPK) analysis of four Phase I, one Phase IIb and one Phase III trials, building a joint compartmental model for pridopidine and TV-45065 in plasma.

Methods: The PPK dataset was constructed by combining rich and sparse PK data from 118 healthy subjects, 24 renalimpaired patients, and 394 HD patients evaluable for PK. The daily doses ranged from 45 to $180 \mathrm{mg}$ and were administered either as a single dose or as multiple doses given once- or twice-a-day. The CYP2D6 genotype or phenotype was assessed in all subjects and the structural PPK model accounted semi-mechanistically for the CYP2D6- and timedependent formation clearance of TV-45065. Age, sex, measures of body size, markers of renal and hepatic function, unit dose of pridopidine and concomitant medications were recorded in the dataset and examined as potential covariates. The final PPK model was qualified by a combination of statistical and graphical diagnostic tools and was the object of a simulation-based internal validation by performing visual predictive checks (VPC).

Results and Conclusion: The PPK model involved linear two-compartment disposition for pridopidine and first-order absorption with a lag-time. The PPK model was able to capture the main characteristics of pridopidine PK: the impact of CYP2D6 polymorphism on the kinetics of pridopidine elimination, and the time-dependent auto-inhibition of CYP2D6. Sex and body weight were identified as additional covariates independently influencing pridopidine clearance. VPCs demonstrated good performance of the PPK model in simulating observed pridopidine concentration time profiles in sub-populations of interest. The model can therefore be 
used to simulate so far untested dosing scenarios and support the design of future pridopidine clinical studies.

\section{POTENTIAL VOLUME AND DIFFUSION BIOMARKERS OF LONGITUDINAL NEUROPATHOLOGICAL CHANGE IN PREMANIFEST AND EARLY SYMPTOMATIC HUNTINGTON'S DISEASE: 30 MONTH DATA FROM THE IMAGE-HD STUDY}

Juan F Domínguez D, Julie C Stout, Govinda R Poudel, Marcus A Gray, Louisa Salmon, Andrew Churchyard, Phyllis Chua, Gary F Egan, Nellie Georgiou-Karistianis Abstract.
Introduction: IMAGE-HD is a single-site longitudinal
biomarker study in premanifest and early Huntington's
disease (HD). Here we present potential biomarkers that
reflect micro- and macro-structural disease related changes over 30 months.

Objectives: We aimed to determine the efficacy with which longitudinal disease progression in HD could be quantified over three testing sessions spanning 30 months using macrostructural (volume) and microstructural (diffusivity) neuroimaging measures. A secondary objective was to evaluate the relationship between longitudinal change volume and diffusion changes and measures of cognitive and psychiatric integrity.

Methods: We analysed longitudinal data collected at baseline, 18 months, and 30 months. Participants included 36 premanifest (pre-HD), 32 symptomatic HD (symp-HD), and 30 healthy control individuals. Clinical, neurocognitive, and neuropsychiatric data were also gathered. Longitudinal group differences in volume and diffusion measures were investigated. The relationship between 30 month change in these measures and long-term neurocognitive and neuropsychiatric status (18 and 30 months average) was also investigated.

Results: Pre-HD and symp-HD groups exhibited greater rates of volume change than controls in grey matter, white matter, cerebrospinal fluid and subcortical structures (caudate, putamen, pallidum and thalamus). Longitudinal group differences were also observed in diffusivity measures, with caudate fractional anisotropy (FA) most consistently discriminating between symp-HD and controls over 18 and 30 months. Effect sizes for significant rates of change between symp-HD and controls were largest for the caudate volume and FA ( $8.4 \%$ and $8.0 \%$, respectively), followed by CSF and pallidum volume $(4.8 \%$ and $4.1 \%$, respectively). In addition, longitudinal caudate loss was most strongly and consistently associated with long-term neurocognitive and neuropsychiatric change in pre-HD close to diagnosis and symp- HD.

Conclusions: A range of imaging biomarker measures could potentially be recommended for clinical trials. We report, with a relatively small sample size, that caudate volume is potentially the most suitable target for treatment as it is the most sensitive to longitudinal change and was associated with a neurocognitive and neuropsychiatric profile.

\section{PREDICTIVE GENE TESTING FOR HUNTINGTON'S DISEASE AND OTHER NEURODEGENERATIVE DISORDERS}

Sarah Wedderburn, Peter K Panegyres, Steve Andrew, Jack Goldblatt, Toni Liebeck, Fran McGrath, Miranda Wiltshire, Carmela Pestell, Joseph Lee, John Beilby

Abstract. The following Abstract was reprinted in part by Miranda Wiltshire with permission from the Publisher IMJ.

Background: The Neurosciences Unit provides specialised services including Predictive Testing to people at risk and living with Huntington's Disease in Western Australia. In May 2013 we published a study (Wedderburn et al., in press*) which explored the controversies which exist around predictive testing (PT) programs in neurodegenerative disorders, including Huntington's Disease (HD).

Aims: This study set out to answer the following questions relating to $\mathrm{HD}$ and other neurodegenerative disorders: differences between these patients in their PT journeys; why and when individuals withdraw from PT; and decision making processes regarding reproductive genetic testing.

Methods: A case series analysis of patients having PT from the multidisciplinary Western Australian Centre for PT over the past 20 years was performed, using Internationally recognized guidelines for predictive gene testing in neurodegenerative disorders.

Results: Of 740 at-risk patients, 518 applied for PT: 466 at risk of HD; 52 at risk of other neurodegenerative disorders: spinocerebellar ataxias, hereditary prion disease and familial Alzheimer's disease. 13\% withdrew from PT $-80.32 \%$ of withdrawals occurred during counselling stages. Major withdrawal reasons related to timing in the patients' lives or unknown as the patient did not disclose the reason. 38 HD individuals had reproductive genetic testing: 34 initiated prenatal testing (of which 8 withdrew from the process) and 4 initiated pre-implantation genetic diagnosis. There was no recorded or other evidence of major psychological reactions or suicides during PT.

Conclusions: People withdrew from PT in relation to life stages and reasons that are unknown. Our findings emphasise the importance of: (i) adherence to internationally recommended guidelines for PT; (ii) the role of the multidisciplinary team in risk minimization; and (iii) patient selection. Acknowledgement: to The Royal College of Physicians and Blackwell Publishing. *International Medical Journal 2013 May 9. doi: 10.1111/imj.12176. [Epub ahead of print] Predictive Gene Testing for Huntington's Disease and Other Neurodegenerative Disorders1 Wedderburn S, Panegyres PK, Andrew S, Goldblatt J, Liebeck T, McGrath F, Wiltshire M, Pestell C, Lee J, Beilby J. 


\section{PREVALENCE OF THE INTERMEDIATE CAG REPEATS IN HUNTINGTON DISEASE. A SPANISH MULTICENTER STUDY OF THE EUROPEAN HUNTINGTON DISEASE REGISTRY}

Esther Cubo, Saul Martinez Indra, Asunción Martin Descallas, Maria Antonia Ramos Arroyo, Spanish EHDN Registry

\begin{abstract}
.
Background: The presence of 36 or more CAG trinucleotide repeats in the huntingtin gene essentially ensures the development of Huntington's disease (HD). However, there is emerging evidence that clinical and neuro-pathological manifestations of HD may occur in individuals with intermediate length CAG repeats (27-35 CAG repeats).

Objetive: To define and compare the clinical characteristics of individuals who possess intermediate CAG repeats with healthy controls.

Methods: Data from the EHDN Registry from Spain were analyzed. We included individuals with intermediate repeats (27-35 CAG ) and controls ( $<27$ CAG repeats). The motor, cognitive and behavioral scores of the Unified Huntington's Disease Rating Scale (UHDRS) were analyzed.

Results: Of 736 participants in the Spanish EHDN Registry, 14 participants, 9 womens [64\%], with a mean age of $50.3+$ 18.2 years with intermediate CAG repeats were identified (prevalence of 1.90, IC 95\% 0.84-2.95), and 77 controls (50 women $[64.9 \%]$ ) with a mean age of $37.3+10.9$ years, were included. Compared to the group of participants with intermédiate $\mathrm{CAG}$ repeats, the control group was younger $(p=0.006)$ with similar scores in the motor, cognitive and behavior UHDRS domains. When the group of participants older than 50 years was selected, only in the group of participants with intermediate CAG repeats, the motor UHDRS score was highly correlated with the age $(r>0.92$, $p<0.008$ ).

Conclusions: In the Spanish HD population, individuals with intermediate CAG repeats in the HTT gen do not show significant clinical manifestations. However, for patients with intermediate CAG repeats, aging might influence the genetic instability and be a risk factor for motor manifestations. These results have important implications for the pathogenesis of the disease and genetic counseling.
\end{abstract}

\section{PRIDOPIDINE IMPROVES HUNTINGTON'S DISEASE IN TRANSGENIC R6/2 MICE}

Alba Di Pardo, Vittorio Maglione, Mariagrazia

Favellato, Enrico Amico, Ferdinando Squitieri

\begin{abstract}
.
Introduction: Huntington's disease (HD) is a neurodegenerative disorder for which new treatments are urgently needed. The disease-causing mutation is an expanded trinucleotide stretch in the huntigtin gene, which translates in an abnormal polyglutamine repeat in the protein product. The disease is characterized by progressive degeneration of brain cells which results in a gradual worsening of behavioral,
\end{abstract}

cognitive and motor function. While there is currently no effective cure for HD, promising research on potential new pharmacological approaches offers new hope. Recent evidence suggests dopaminergic circuitry as a potential therapeutic target to ameliorate the symptoms of the disease. A new dopaminergic stabilizer, pridopidine, has been recently developed for the treatment of motor symptoms associated with HD. Results has indicated improvement of total motor function after pridopidine treatment in two double-blind, randomized phase II (HART study) and phase III (MermaiHD study) clinical trials in patients with HD. A 6 months openlabel extension to the MermaiHD study showed long-term tolerability and safety profile of the drug.

Objective: To validate the symptomatic benefit of pridopidine in HD models in order to explore additionally the potential of neuroprotection and eventually disease-modifying properties of the drug.

Methods: HD transgenic R6/2 mice and wild type littermates were chronically administered with pridopidine and, the effect on motor function and longevity was assessed by behavioral testing and survival analysis, respectively. Potential neuropathological changes were analyzed by immunohistochemistry.

Results: In line with clinical studies, administration of pridopidine efficiently ameliorated the overall motor impairment and improved coordination in R6/2 mice. The drug additionally significantly prolonged lifespan and induced appreciable neuropathological changes in mice by increasing striatal expression of BDNF and DARPP32 and promoting neuronal connectivity.

Conclusions: The effect of pridopidine on motor function in HD mice recapitulated the symptomatic benefits observed in HD patients. The validation of drug efficacy in HD mice may be a usefull tool to better understand how pridopidine exert effects in human brains. Our findings suggest that the improved motor function may depend on neuroprotective effect of pridopidine, and propose it as a concrete possibility to treat HD.

\section{REPORTED EXPERIENCE DURING THE FIRST YEAR OF A MULTIDISCIPLINARY HUNTINGTON'S CLINIC AT SANT PAU HOSPITAL IN SPAIN}

Jesus Perez Perez, Saul Martinez-Horta,

Mar Carceller Sinderu, Ramon Fernandez de

Bobadilla, Javier Pagonabarraga,

Antonia Campolongo, Berta Pascual Sedano, Jaime Kulisevsky

\section{Abstract.}

Introduction: The clinical presentation of Huntington's disease (HD) includes motor, behavioral and cognitive symptoms that require the intervention of different specialists. Clinical management without reaching consensus between specialists supposes higher probability of discrepancies on the diagnosis and treatment, increasing the risk for secondary effects, worse prognosis and to neglect needs like physiotherapy, nutrition and/or psychological support.

Objectives: To analyze the clinical activity and viability of a multidisciplinary HD clinic during its first year. 
Methods: Prospective registry of the data obtained from the visits done by the team composed by a neurologist, psychiatrist, neuropsychologist, nurse, physiotherapist and nutritionist. Identification of type, reason for the visit and specialists involved. Comparison between total numbers of admissions did before the consolidation of the HD clinic and during the first year of it in a same time period.

Results: Analysis of visits from 65 patients (40 first visits; $65.5 \%, 25$ follow-up visits; $28.5 \%$ ). For the first visits, 31 cases $(50.8 \%)$ were genetic counseling and 7 cases $(11.5 \%)$ de-novo diagnosis ( $3 \%$ Huntington-like). $96.7 \%$ of the patients were seen by the neurologist, 95.1 by the neuropsychologist, $81.3 \%$ by the psychiatrist, $41 \%$ by the nurse, $22.3 \%$ by the physiotherapist and $11.4 \%$ by the nutritionist. The specialties requiring more visits were psychiatry (up to 5 visits/year) and neurology (up to 4 visits/year) followed by neuropsychology, nutrition and physiotherapy (up to 2 visits/year). Decision making on treatment and follow-up schedule were done after agreement between HD clinic members for all the patients. Number of admissions during 2011-2012 was six and diminished to 2 after the creation of the HD clinic.

Conclusion: Given the characteristics of HD, multidisciplinary HD clinics are extremely recommended for an optimum management of HD. This model improves the quality of the medical care for the patients/families and reduces the number of admissions.

\section{REVERSAL LEARNING IS IMPAIRED IN HUNTINGTON'S DISEASE}

Katharina Nickchen, Rebecca Böhme, Maria del Mar Amador, Katharina Gisske, Patricia Panneck, Lorenz Deserno, Florian Schlagenhauf, Josef Priller

Abstract. Huntington's disease (HD) is a rare autosoma dominant neurodegenerative condition that commonly presents in mid life with a combination of a movement disorder, psychiatric and cognitive problems. $\mathrm{HD}$ is characterized by neuronal dysfunction, shrinkage and ultimately loss in a wide range of brain structures. The caudate and putamen appear to be particularly vulnerable and show significant atrophy even in presymptomatic stages. Given that recent evidence has implicated ventral prefrontostriatal circuits in reward- and punishment-based learning, studies in HD may contribute to a better understanding of the role of the striatum in flexible learning. We tested the performance of 20 manifest HD patients in various disease stages and all without symptomatic medication in a reversal learning task using functional magnetic resonance imaging (fMRI). We also performed detailed motor, psychiatric and neuropsychological evaluations of the patients, and compared the results with ageand sex-matched healthy controls. Computational modelling of reinforcement learning was used to describe changes in learning dynamically and to provide quantitative fits and parameters for individual behaviour. In healthy controls, the behavioural performance correlated well with reward sensitivity. In contrast, the behaviour of HD patients was more influenced by their sensitivity to punishments. Despite of significant structural changes in the basal ganglia of HD patients, the behavioural deficits so far were not associated with decreased blood oxygen level-dependent (BOLD) activity in the ventral striatum. Our data suggest that HD patients are not able to maintain an advantageous behaviour in the reversal learning task because punishments, which occur as probabilistic events, seem to have a stronger influence on their choices than a generally reduced sensitivity to past rewards. We are currently analyzing the fMRI data with respect to switch-stay behaviour, and we are trying to disentangle the relationship of functional activation during learning with the degree of structural neuropathological changes. This will provide insight into correlations between basal ganglia abnormalities and reinforcement learning.

\section{SAFETY AND EFFECTIVENESS OF LITHIUM CARBONATE FOR THE TREATMENT OF MACHADO-JOSEPH DISEASE: A PHASE 2/3 RANDOMISED, DOUBLE-BLIND, PLACEBO- CONTROLLED TRIAL}

Jonas Alex Morales Saute, Raphael Machado de Castilhos, Thais Lampert Monte, Artur Francisco Schumacher Schuh, Karina Carvalho Donis, Rui D’Ávila, Gabriele Nunes Souza, Aline Dutra Russo, Gabriel Vasata Furtado, Tailise Conte Gheno, Diogo Onofre Gomes de

\section{Abstract.}

Background: MJD/SCA3 is caused by a CAG repeat expansion (CAGexp) at ATXN3 gene, leading to progressive loss of motor coordination that is current untreatable. Lithium was shown to exert neuroprotective effects in pre-clinical models of polyglutamine disorders. We aimed to assess safety and effectiveness of lithium carbonate $(0.5-0.8 \mathrm{mEq} / \mathrm{L})$ in patients with MJD/SCA3. Methods: A phase 2/3 single center, double-blind, parallel, placebo-controlled trial was conducted (ClinicalTrials.gov,NCT01096082). Patients, caregivers, and all study staff, but pharmacists and principal investigators, were masked to treatment assignment. Between May and September, 2011, 62 independently ambulatory MJD/SCA3 patients with $\leq 10$ years of disease duration were randomly assigned (1:1, stratified by CAGexp) to lithium $(0.5-0.8 \mathrm{mEq} / \mathrm{L})$ or placebo. Primary safety end-point $(24$ weeks) was the difference in the total number of adverse events (AE) and of effectiveness (48 weeks), the difference in the variation of NESSCA scale between groups. Analysis was by intention-to-treat (ITT).

Results: Sixty patients (31/29 placebo/lithium) were analysed according to ITT. After 24 weeks, 169 AE were reported, $50.3 \%$ in lithium group $(p=1.00)$. After 48 weeks, placebo group had a non-significant larger progression on NESSCA ( 0.35 points, $95 \%$ CI -1.0 to $1.7, p=0.222)$ and SARA scales ( 0.96 points, $95 \% \mathrm{CI}-0.46$ to $2.38, p=0.329$ ). Lithium group had significantly slower progression after 48 weeks in PATA rate $(p=0.002)$, Click Test ND $(p=0.023), \operatorname{SCAFI}(p=0.015)$ and CCFS $(p=0.029)$.

Conclusion: Lithium is safe and well tolerated, but had no significant effect on NESSCA progression of MJD/SCA3. In contrast, lithium significantly slowed the progression of several secondary outcomes all related to ataxic manifestations. These results open new perspectives for the treatment of MJD/SCA3 and other polyglutamine disorders that must be confirmed in larger multicenter clinical trials. 


\section{SEARCHING FOR GENETIC MODIFIERS OF AGE AT ONSET IN HUNTINGTON'S DISEASE}

\author{
Aline Francielle Damo Souza, Tailise Conte Gheno, \\ Gabriel Vasata Furtado, Raphael Machado de \\ Castilhos, Carlos Roberto de Mello Rieder, \\ Laura Bannach Jardim, Maria Luiza Saraiva-Pereira
}

\begin{abstract}
Huntington disease (HD) is a progressive disorder of motor, cognitive, and psychiatric functions caused by CAG repeats expansion in the HTT gene. Sixty to seventy percent of age at onset (AO) is explained by CAG repeats length of the mutant HTT allele; the remaining variation being probably due to genetic and environmental factors. Some genetic modifiers of AO have already been described, such as HAP1 gene. The aim of this study was to assess specific polymorphisms in GRM1, SGK1, and TGM2 genes, which are candidate genes to modifiers, and HAP1 gene and correlate with $\mathrm{AO}$ of patients with HD. Our sample population includes 91 unrelated HD patients and 100 unrelated healthy subjects as controls. DNA was isolated from peripheral blood by standard methods. The majority of SNPs were selected using SNP Tagger tool of HAPLOVIEW software. rs4523977 (HAP1) and rs6923492 (GRM1) were included based on previously published data. Allelic discrimination of SNPs was performed by qualitative real time PCR using the TaqMan ${ }^{\circledR}$ PCR Assay. Results were analyzed by the SDS Software version 1.2.1 in the ABI Prism 7500 through allelic discrimination plot. Statistical analyses were performed using SPSS Statistics v.18. Regression model was used to assess the influence of rs1331639 (GRM1), rs1763505 (SGK1), rs7270898 (TGM2), rs7275079 (TGM2), and rs4523977 (HAP1) in AO variation together with length of CAG tract. Each SNP genotype was assessed one by one. In our sample population mean $\mathrm{AO}$ was 37.3 years ( \pm 12.4 years) ranging from 6 to 72 years. CAG repeat length was responsible for $65.3 \%\left(R^{2}=0.653 ; p<0.0001\right)$ of variation in AO. A protective effect was observed in rs4523977 (HAP1), with a delay in AO of patients with AA genotype $(p=0.044)$ when compared to other possible genotypes. This finding confirms previously published data. We have not observed significant effect of the other SNPs included in this study. HAP1 is involved in axonal traffic that interacts with huntingtin (htt), being selectively expressed in neurons. Functional studies published previously show that HAP1 protein produced by the A allele of the gene interacts with mutant htt more tightly than does HAP1 produced by the $\mathrm{T}$ allele, reduces soluble htt degraded products and protects against htt-mediated toxicity. Therefore, the effect observed in this study is the result of all this functions in htt metabolism. (Financial support: CNPq, FAPERGS \& FIPE-HCPA).
\end{abstract}

\section{SELF-DUPLEXING CUG REPEATS \\ SELECTIVELY INHIBIT MUTANT HUNTINGTIN EXPRESSION}

Wlodzimierz J. Krzyzosiak, Agnieszka Fiszer, Marta Olejniczak, Paulina Galka-Marciniak, Agnieszka Mykowska

\section{Abstract.}

Introduction: Huntington's disease (HD) is a neurodegenerative genetic disorder caused by the expansion of the CAG repeat in the translated sequence of the HTT gene. This expansion generates a mutant huntingtin protein that contains an abnormally elongated polyglutamine tract, which together with mutant transcript causes cellular dysfunction.

Objective: Currently, there is no causative treatment available to patients suffering from $\mathrm{HD}$, and our study was aimed at the selective inhibition of the mutant allele expression which is a promising therapeutic option.

Method: We used unmodified oligoribonucleotides to target mutant HTT allele in patient-derived cell lines, and the assays performed included transcript analysis by RT-PCR and protein analysis by western blotting

Results: We developed a new class of CAG repeat-targeting silencing reagents that consist of self-duplexing CUG repeats. Self-duplex formation was induced through one or several U-base substitutions. A number of self-duplexing guidestrand-only siRNAs have been tested and the best reagents were highly discriminatory between the normal and mutant HTT alleles and the HTT transcript and other transcripts containing shorter CAG repeats. We also demonstrated that the self-duplexing CUG repeat siRNAs use the RNAi pathway to elicit silencing, and repeat targeting reagents showed similar activity and selectivity when expressed from shRNA vectors to achieve more durable silencing effects.

Conclusion: The self-duplexing CUG repeats are selective triggers of mutant HTT silencing, having potential for therapeutic applications.

\section{SEXUAL DIFFICULTIES IN HUNTINGTON'S DISEASE. WHAT ARE THE NEEDS FOR COUNSELING COUPLES?}

Birgitta Hulter

\section{Abstract.}

Background: Huntington's Disease creates various physical, mental, and social disabling impairments that will most likely affect sexuality and intimate relationships. Up to $85 \%$ men and $75 \%$ women experience high levels of sexual problems (Schmidt \& Bonelli, 2007). Most prevalent is low sexual desire, but increased sexual interest, and disturbing behaviors can also occur. Impaired self-awareness of cognitive, emotional, and functional deficits creates complications in communicating and interacting sexually. Specific brain lesions might be associated with these changes, but also psychosocial factors with a worsening disease might be causative. Depression and anxiety, common symptoms in early stages of HD, are often associated with sexual symptoms. Change in sexual interest and behavior, sexual dysfunction, 
and socio-psychiatric consequences of HD cause suffering for patients, spouses and children. Behavioral problems may be most distressing and disrupting for family members.

Aims: Because intimate issues and sexuality in most places are taboo topics, the responsibility, and the initiative for opening up the conversation, lies on the health professional/ physician, meeting the couple. To promote wellbeing to patient, partner, and children, sincere talks on relational and sexual issues may add great value in the couple.

Methods/Techniques: Bio-psycho-social views can be applied in support and rehabilitation in couples with chronic neurological impairments. Talks follow the basics of the PLISSIT model (Annon, 1976). Acceptance and Commitment Therapy is a treatment option.

Results/Outcome: A comprehensive management and treatment of sexual issues in HD might be needed. Training and experience in sexology should be mandatory for professionals in counseling and support.

Conclusions: HD brings suffering in connection to sexuality. Sexual problems often reduce life satisfaction in patients and partners. The whole family situation is affected. Professional attention should be given to the sexual symptoms in HD. Clinical sexology knowledge and skills should be improved. Sexological rehabilitation requires a broad focus on bodily changes, identity matters, as well as relationship challenges and adjustment.

\section{SIX-MONTH CHANGE IN NEUROIMAGING, CLINICAL AND COGNITIVE MEASURES IN STAGE 1 HUNTINGTON'S DISEASE: PREPARING FOR CLINICAL TRIALS}

Nicola Z Hobbs, Ruth E Farmer, James H Cole, Elin M Rees, Salman Haider, Reiner Sprengelmeyer, HansPeter Mueller, Sigurd D Sussmuth, Raymund AC Roos, Alexandra Durr, Chris Frost, Rachael I Scahill, Bernhard Landwehrmeyer, Sarah J Tabrizi

\footnotetext{
Abstract.

Introduction: Biomarkers capable of quantifying disease progression over short time intervals are urgently needed to facilitate drug development and increase the viability of clinical trials in neurodegenerative diseases such as Huntington's disease (HD).

Objectives: To evaluate a range of biomarker candidates for HD over a 6-month interval. To utilise a large well-defined cohort of stage $1 \mathrm{HD}$, with a multi-national, multi-site study design, representative of the population and set-up required for disease-modifying clinical trials.

Methods: 40 controls and 59 stage $1 \mathrm{HD}$ subjects, recruited from four sites across the European Union as part of the PADDINGTON Study, underwent 3T MRI (T1-, T2-, diffusion-weighted), plus a standard clinical and cognitive battery at baseline and 6 months. Neuroimaging measures included longitudinal change in macrostructure (volume) and microstructure (fractional anisotropy, mean-, radial-, and axial- diffusivity) over pre-defined regions-of-interest. Group differences in 6-month change were examined, adjusting for potential confounders. Six-month effect sizes (ES) were estimated.
}

Results: Over 6 months stage 1 HD showed significant decline in 3/11 cognitive measures (Hopkin's Verbal Learning Task, Symbol Digit Modality Task, Trails A), and the UHDRS Total Functional Capacity score (all $p<0.05$ ). There was no evidence of decline in motor performance according to the UHDRS Total Motor Score (TMS). Macrostructural neuroimaging measures showed significantly elevated brain atrophy rates in HD, both globally (whole brain, ventricles, white matter (WM), grey matter (GM)) and in the caudate (all $p<0.01$ ), but not the putamen or corpus callosum. Microstructural neuroimaging measures were more variable with 6/16 measures showing significant change over 6 months. The largest 6-month ES were for macrostructural metrics: volume change in the caudate $(0.795,95 \%$ CI: 0.393 , $1.138)$, ventricles $(0.702,95 \%$ CI: 0.2081 .110$)$, WM (0.721, $95 \%$ CI: 0.2931 .162$)$ and GM $(0.707,95 \%$ CI: 0.0331 .162$)$. These were significantly larger than ES for TMS and 3 cognitive measures $(p<0.05)$.

Conclusions: Macrostructural neuroimaging measures in stage $1 \mathrm{HD}$ appear particularly powerful over short time intervals, compared with standard clinical and cognitive scales. They may provide valuable secondary endpoints for short proof-of-concept clinical trials of putative diseasemodifying compounds and data to inform the design of Phase 3 trials.

\section{SOLUBLE N-TERMINAL FRAGMENT OF MUTANT HUNTINGTIN PROTEIN IMPAIRS MITOCHONDRIAL AXONAL TRANSPORT IN CULTURED HIPPOCAMPAL NEURONS}

Baorong Zhang, Jun Tian

\section{Abstract.}

Objective: To investigate the different impacts of soluble and aggregated N-terminal fragments of mutant Huntingtin protein $(\mathrm{mHtt})$ on mitochondrial axonal transport in cultured hippocampal neurons.

Methods: Primary cultured hippocampal neurons were cotransfected with pDsRed2 and GFP-Htt/GFP-mHtt/GFP. Mitochondrial axonal transport was monitored by live imaging.

Results: We found that the N-terminal fragment of $\mathrm{mHtt}$ formed aggregates in $44 \pm 2.87 \%$ of transfected hippocampal neurons. Overexpression of $\mathrm{N}$-terminal fragment of $\mathrm{mHtt}$ decreased mitochondrial axonal transport velocity and mitochondrial mobility in hippocampal neurons regardless of whether aggregates were formed. However, impairment of mitochondrial axonal transport between neurons expressing the soluble and aggregated $\mathrm{N}$-terminal fragments of $\mathrm{mHtt}$ did not differ.

Conclusions: Our findings indicate that both the soluble and aggregated $\mathrm{N}$-terminal fragments of $\mathrm{mHtt}$ impaired mitochondrial axonal transport in cultured hippocampal neurons. We predicted that mitochondrial axonal transport dysfunction could be an early-stage event in the progression of $\mathrm{HD}$, even before $\mathrm{mHtt}$ aggregates are formed. 


\section{STATEWIDE COLLABORATION BETWEEN A CORRECTIONAL SYSTEM AND A HUNTINGTON'S DISEASE CLINICAL PROGRAM}

\author{
Bonnie L. Hennig, Robert L. Trestman
}

\begin{abstract}
.
Introduction: One of the impairments associated with Huntington's Disease (HD) is disinhibition of behavior and risky decision making (Stout et al 2001). This can result in activity leading to incarceration (Jepsen et al., 1998). A major challenge is ongoing care coordination to optimize treatment, maintain community linkages during incarceration, and ensure successful community reintegration upon release. A model statewide program successfully addressing these challenges has been developed and implemented.

Objectives: Describe the components of a statewide collaboration between a correctional system and a HD clinical program, the process of its implementation, and share illustrative case studies.
\end{abstract}

Methods: The University of Connecticut Health Center (UCHC) hosts a statewide HD clinical program, responsible for providing care to people with $\mathrm{HD}$, those at-risk for developing HD, and their families. The UCHC HD program has worked for several years with Correctional Managed Health Care (CMHC), a division of $\mathrm{UCHC}$ responsible for all health care for those incarcerated in Connecticut's jails and prisons. The UCHC HD program has developed active linkages for CMHC staff education, information sharing (especially regarding medication management), transport of patients to the clinic from custody for ongoing care consultation, and close coordination with CMHC Transition Managers for community reentry planning.

Results: UCHC HD patients who have become incarcerated have their care maintained without disruption, are able to consult with their community-based care team, and the inevitable challenges of successful community reentry are optimized with the shared skill sets and knowledge of two teams (correctional and community).

Conclusion: Illegal, impulsive behavior of HD patients is not common. However, when it occurs it can seriously disrupt optimal care. The joint program developed in Connecticut may serve as a generalizable model to facilitate care continuity for HD patients who become incarcerated.

References: Jensen, P., Fenger, K., Bolwig, T.g., Sorensen, S.A. (1998). Crime in huntington's disease: a study of registered offences among patients, relative, and controls. Journal of Neurology Neurosurgery, \& Psychiatry: 65(4): 467-471.

Stout, J.C., Rodawalt, W.C., \& Siemers, E.R. (2001). Risky decision making in huntington's disease. Journal of the International Neuropsychological Society: JINS, 7(1), 92-101.

\section{TEST-RETEST RELIABILITY OF THE ASESSMENT OF MOTOR AND PROCESS SKILLS (AMPS) IN HD}

\author{
Rebecca Devlin, Monica Busse, Clare Cook, \\ Hugh Rickards
}

\begin{abstract}
.
Introduction: It is important that clinicians measure the functional ability of people with HD over a period of time due to $\mathrm{HD}$ resulting in a gradual deterioration in functional capacity. Functional outcomes are a vital part of clinical trials especially in relation to drug licencing. Literature has stated the importance of measuring functional levels within HD in the development of medications. Current measures of function used in HD are not based on observation of task performance and are more frequently self-reported measures which have poor validity in comparison to standardised observational assessments. The Assessment of Motor and Process Skills (AMPS) is an assessment tool used by occupational therapists worldwide to assess the functional skills of an individual by observing them perform two familiar and relevant activities of daily living (ADLs). The AMPS has been validated in various neurological conditions, however, to date its validity in HD has not been investigated. This research aims to establish: 1. test-retest reliability of the AMPS in people with HD. 2. concurrent validity of the AMPS in relation to other assessments used in REGISTRY.
\end{abstract}

Method: To date 14 participants have been recruited, 9 males and 5 females aged between 39 and 70 years old. Participants are recruited at time of their REGISTRY visits. AMPS evaluations take place in participants' homes (time point 1) and repeated within 14 days (time point 2). Scores from the UHDRS motor, cognitive and functional assessments are completed up to 14 days prior to the baseline AMPS (time point 1) and will be used for correlative analysis.

Results: Data collection is still on-going. Current analysis using t-tests indicates that there is no significant difference between the AMPS scores at time point 1 and time point 2 . Mean scores for AMPS Scores at Time point 1 and Time point 2 (95\% CI for difference and $\mathrm{p}$ value) Time point 1 Time point $2 p$ value AMPS Motor Score $1.33(0.89) 1.26$ (0.87) $p=0.33$ AMPS Process Score 1.09 (0.55) 1.03 (0.56) $p=0.17$ Initial results appear to support the hypothesis that the AMPS has good test-retest reliability. Further analysis is needed to assess concurrent validity.

Discussion: Based on the results so far, the findings support the need for further research to be conducted into the use of AMPS in the HD population. 


\section{THE HUMAN GLOBUS PALLIDUS IN HUNTINGTON'S DISEASE: CHARACTERIZATION OF VOLUMETRIC CHANGES, PALLIDAL NEURON LOSS AND CELL MORPHOLOGY IN ASSOCIATION WITH PATHOLOGICAL GRADE AND SYMPTOM-TYPE}

Malvindar Singh-Bains, Virginia Hogg, Beth Synek, Lynette Tippett, Henry Waldvogel, Richard Faull

\author{
Abstract. \\ Introduction: In Huntington's disease (HD) numerous \\ studies have documented the patterns of cell loss in the \\ striatum. However, there are no studies using design-based \\ stereology to assess the impact of HD on a principle target of \\ striatal outflow, the globus pallidus (GP).
}

Objectives: To characterize regional volume, pallidal neuron loss and morphological changes in both segments of the HD human globus pallidus using design-based stereology; and explore the relationships between these data and HD striatal neuropathological grade, and clinical symptomatology.

Methods: In the present double-blind study, $8 \mathrm{HD}$ and 8 normal cases were analysed using the optical fractionator to measure the volume of the globus pallidus, and quantify pallidal neurons. Pallidal neurons were identified on Nisslstained sections, with external (GPe) and internal (GPi) boundaries delineated with enkephalin and substance-P immunoreactivity. The cross sectional areas of parvalbuminpositive pallidal neurons were measured using the isotropic nucleator. Pathology and symptomatology data of HD cases were collected, with reference to the Quantitative Neurological Exam (QNE) and Unified Huntington's Disease Rating Scale (UHDRS).

Results: Stereological analysis reveals the HD GPe reduces to more than half the normal volume, with quantification showing $>60 \%$ pallidal neuron loss. The HD GPi volume is also reduced, to a smaller degree compared to the GPe, with minor cell loss (20\%). Morphometric analysis show that HD GPe pallidal neurons were $35 \%$ smaller compared to normal, with no changes in the GPi. Changes in regional volume and cell loss were shown to relate to striatal pathology grades, with greater volume reduction and cell loss corresponding with increased grades. Preliminary analysis shows that there is a strong correlation between HD GPe volumes and both QNE/UHDRS motor impairment score severity, but not with chorea.

Conclusion: This study highlights that the GPe is more vulnerable to $\mathrm{HD}$ than the GPi, consistent with neurochemical studies showing that enkephalinergic striatal-GPe fibres degenerate in advance of substance-P striatal-GPi fibres. In particular, our studies show that GPe atrophy is related to HD clinical symptom-type, with regional atrophy showing a strong correlation with the severity of motor impairment, but not with chorea. Support: Health Research Council of New Zealand; Neurological Foundation of New Zealand; The University of Auckland.

\section{THE HUNTINGTON'S DISEASE COGNITIVE ASSESSMENT BATTERY (HD-CAB), A STANDARDISED COGNITIVE BATTERY FOR CLINICAL TRIALS}

\author{
Julie C. Stout, Sarah Queller, Kalyca Baker, \\ Sean Cowlishaw, Beth Borowsky
}

\begin{abstract}
.
Aim: To describe the development, characterisation, and standardisation of HD-CAB, a cognitive battery for clinical trials and clinical research in Huntington's disease.

Methods: We evaluated a battery of 15 tests selected from published literature and based on expert advice. The battery was administered three times, twice on consecutive days, and then once six weeks later, in order to simulate practice effects, reliability and feasibility relevant to clinical trial conditions. The 263 participants included 105 controls, 103 late premanifest, 55 early HD, all English speaking, at 20 sites in Australia, the US, Canada, and the UK. Effect sizes (Cohen's d) comparing clinical (premanifest or HD) to control group were used to characterise sensitivity to group differences. Reliability was determined using Pearson correlations across adjacent visits. Feasibility and tolerability were assessed using examiner ratings and test administration times. Practice effects were computed as paired standardised mean differences between adjacent visits. We then selected 7 tests for HD-CAB, based on broad coverage of relevant cognitive domains, sensitivity in late premanifest and early stage HD, and strong psychometric properties.

Results: The HD-CAB includes 7 tests with demonstrated sensitivity to disease status (dEarlyHD $=1.59-2.16$; $\mathrm{dPreHD}=0.60-0.83$ ) and good reliability (r's 0.73-0.93), and acceptable tolerability and feasibility. Practice effects were evident at the second time point but were generally less evident at the third time point. The HD-CAB composite had large effect sizes $(\mathrm{dEarlyHD}=2.78$ and $\mathrm{dPreHD}=1.17)$ and high reliability $(r=0.93)$. The median HD-CAB administration time was estimated at 33 minutes in Early HD.

Conclusions: The HD-CAB assesses a range of cognitive functions, is sensitive to HD status, reliable, tolerable, and pragmatically feasible in the context of HD clinical trials. Preexposure to the HD-CAB tests prior to the baseline visit is
\end{abstract} recommended to reduce practice effects.

\section{THE HUNTINGTON'S DISEASE DYSPHAGIA SCALE (HDDS) FOR MEASURING DYSPHAGIA IN HUNTINGTON'S DISEASE PATIENTS}

Anne-Wil Heemskerk, Berit Verbist, Han Marinus, Bas Heijnen, Elizabeth Sjogren, Raymund Roos

\footnotetext{
Abstract.

Background: Many patients with Huntington's disease (HD) have swallowing problems. These problems may lead to aspiration pneumonia, which is the most frequently encountered primary cause of death in HD. Little is known about the occurrence, severity, and progression of swallowing disturbances in HD. Better insights into the prevalence and course of dysphagia, may lead to an increased awareness and
} 
a more appropriate treatment that is tailored to the patients individual needs. The goal of our study was to develop and validate the Huntington's Disease Dysphagia Scale (HDDS), an instrument that can be used to determine the incidence of dysphagia in HD, and allows monitoring of the severity of this symptom over time.

Methods: The scale was developed in four stages: 1) item generation, based on a literature search and on input by a team of experts; 2) item comprehensibility testing; 3) evaluating reliability in HD patients and their relatives; 4) item reduction and scale construction, followed by examining its construct validity by comparison with the Swallowing Disturbance Questionnaire (SDQ).

Results: For the HDDS, the expert team identified 14 questions of clinical relevance. Next, $55 \mathrm{HD}$ patients ( 24 men, mean age $53 \pm 12$ years) in the three clinical stages of HD participated in the study. The intra-rater reliability of one question ('wet voice after swallowing') was very low $(\mathrm{ICC}=0.189)$ and was therefore removed. Cronbach's alpha for the 13 remaining questions was 0.670 . Cronbach's alpha increased further to 0.728 after removal of the items on 'residue in the mouth, cheek or teeth' and 'producing sounds during eating or drinking'. Finally, 11 items were included in the HDDS. The correlation with SDQ (Pearson's correlation coefficient) was 0.734 .

Conclusions: We developed an 11-item scale to measure dysphagia in HD. The instrument can be used to screen and monitor swallowing difficulties from early manifest to endstage disease. The final questionnaire, which will be shown on the poster, demonstrated good reliability and construct validity.

\section{THE IMPACT OF CAG REPEAT LENGTH IN THE HUNTINGTIN GENE ON CHILDHOOD NORMAL BRAIN STRUCTURE AND FUNCTION}

Jessica K. Lee, Elizabeth D. Mills, Amy L. Conrad, Jeffrey D. Dawson, Eric A. Epping, Kathy Mathews, Peg C. Nopoulos

\footnotetext{
Abstract.

Introduction: Simple sequence repeats such as CAG trinucleotide repeats are regarded as a source which provides normal variation in the genetic trait they are associated with. The huntingtin gene (HTT) causes a degenerative brain disease, Huntington's Disease (HD), when the CAG repeats are expanded beyond 36. Conversely, HTT has been shown to be vital for neuronal development, and therefore variance in $\mathrm{CAG}$ repeat length within this gene, below disease threshold, may be linked to the variability of the structure and function of the normal brain.

Objective: The current study examines the influence of CAG repeat length in HTT on structural and functional modification of the normal brain.

Methods: Standardized measures of behavior, motor and cognitive function, as well as MRI scans were obtained from 43 children (ages 6-18 years). All of the participants came from HD families, however, were tested (for research purposes only) to have $\mathrm{CAG}$ repeats within the normal range
}

$(\mathrm{CAG}$ repeats $<36$ ). The contribution of $\mathrm{CAG}$ repeat length on functional measures and brain structure were estimated. Results: Children with longer CAG repeats, yet still below disease threshold, showed 1) fewer aggression/opposition, hyperactivity/inattention and depression/anxiety problems as measured by Pediatric Behavior Scale (PBS) and 2) better behavior regulation abilities as measured by Behavior Rating Inventory of Executive Function (BRIEF). The results of Physical and Neurological Examination for Subtle Signs (PANESS) revealed longer CAG repeats to be associated with better performance in timed repetitive and sequential tasks. We observed an increase in cerebellum and decrease in putamen volume with increasing CAG repeat length, which were again associated with better behavior regulation and faster speeded performance, respectively. Conclusions: The results suggest that increase in CAG repeat length within the normal range mediates advantageous changes of structure and function of the brain.

\section{THE POTENTIAL ROLE OF NEUROPSYCHOLOGICAL AND SACCADIC MEASURES IN DEFINING DISEASE PROGRESSION AND SEVERITY IN HUNTINGTON'S DISEASE}

\section{Filipa Júlio, Gina Caetano, Cristina Januário, Miguel Castelo-Branco}

Abstract. Huntington's disease (HD) is a genetic neurodegenerative disorder that primarily affects the basal ganglia and the fronto-striatal circuitry. Oculomotor abnormalities have been described as one of the earliest symptoms presented by HD patients and have also been reported in premanifest gene carriers. This study aims to understand the role of saccades as a potential biomarker of disease progression and severity. A comprehensive battery of neuropsychological tests was used to assess the overall cognitive functioning of the participants: 12 early HD patients, 11 premanifest HD subjects (preHD) and 15 healthy controls. An experimental paradigm was designed to assess oculomotor function. Participants had to complete four different horizontal saccadic tasks: prosaccade, antisaccade, 1-or-2 back memory prosaccade and 1-or-2 back memory antisaccade. Data were recorded using an iViewX high-speed eye tracker. Success rate (percentage of error free trials), directional and timing errors were calculated for each task. Early manifest non-demented HD patients exhibited deficits in several of the neuropsychological tests applied, namely in visual and verbal memory, executive function, attention, visual perception and verbal and non-verbal IQ domains. No significant differences were found between preHD and control subjects in any of these measures, suggesting a similar cognitive baseline. Statistically significant differences were found between the manifest HD group and the preHD and control groups in several of the computed saccadic parameters. HD patients showed a significant decrease in the success rate and a significant increase in the percentage of timing and directional errors. A marginal difference was found between preHD and control groups in the percentage of long latency errors, possibly indicating a different strategy for successful task completion during executive/memory load in the latter. 
These preliminary results suggest that the performance of early manifest and preHD participants deteriorate when an executive or/and memory load is added to the task. Moreover, the clinical groups appear to have deficits in goal oriented oculomotor behavior - with a trend of more automatic responses at the cost of timely decision-making. These data suggest that temporal saccade properties under distinct working memory loads can potentially mark the presence of early neurodegeneration in $\mathrm{HD}$ and could serve as quantitative measures of disease progression and severity.

\section{THE RELATIONSHIP BETWEEN DIETARY INTAKE AND HUNTINGTON'S DISEASE SEVERITY}

Esther Cubo, Jessica Rivadeneyra, Diana Armesto, Ana Mateos, Rafael Camara, Asuncion Martinez Descalls, Cecilia Gil, Spanish European HD Registry

\section{Abstract.}

Background: Weight loss is common among patients with Huntington's disease (HD), although the mechanisms contributing to this phenomenon are not well known.

Objective: to analyze the relationship between dietary intake and HD severity.

Methods: National, multicenter, cross-sectional study (Registry EHDN). The dietary intake in terms of caloric and nutrients intake at an individual and population level in presymptomatic and symptomatic patients with HD were assessed using validated questionnaires for the Spanish population and the Spanish Dietary Recommended Intakes (SDRI). In addition, nutritional state was estimated by the body mass index (BMI), disease severity using the Unified HD Rating Scale (UHDRS) and quality of life using the SF36 questionnaire.

Results: One hundred and twenty eight patients with HD were included ( $54 \%$ women) with a mean age of 48.8 years + 14.1, mean CAG repeats of $44.0+5.6$, median Total Functional Capacity (TFC) of 6.5 (IQR 10.7), and median UHDRS motor score of 42 (IQR 53). In this population, 63\% had lower caloric intake compared to the SDRI for specific age-and gender Spanish healthy groups. This sample had a higher intake of proteins, total fat, vitamins C, A, and B12, selenium, thiamine, riboflavin, niacin, pantothenic acid, pyridoxine, biotin, phosphorus, and potassium, and normal monounsaturated/polyunsaturated fat ratio, according to the SDRI. According to the World Health Organization classification, only $7 \%$ were underweight, $58.6 \%$ had normal weight; $24.2 \%$ were overweight, and $10.2 \%$ were obese. CAG repeat lengths were not significantly correlated with BMI or caloric intake. Older patients ( $>55$ years old) and high CAG repeats $(>40)$ had similar BMI and caloric intake compared to the rest of the patients. Compared to patients with normal or higher caloric intake, patients with lower caloric intake were more depressed $(p=0.008)$, but had similar age, motor, cognitive, TFC, caregiver burden, and quality of life. Patients with abnormal BMI had higher motor-UHDRS scores compared to those with normal BMI $(p=0.03)$, and were similar in other clinical aspects.

Conclusions: In this ongoing study conducted in the Spanish HD population, our diet was mostly associated with normal weight. However, patients with depression and severe motor impairment are at higher risk for nutritional imbalance.

\section{THE ROLE OF THE HUNTINGTON'S DISEASE NURSE SPECIALIST IN IDENTIFYING THE NEEDS AND CO-ORDINATING INDIVIDUALISED CARE PACKAGES FOR HD FAMILIES; INCLUDING THOSE AFFECTED, GENE POSITIVE, AT RISK AND CARERS}

Jo Dysart, Greg Finucane, Jane Devine, Richard Roxburgh, Richard Faull, Melanie Cheung

Abstract. The needs of different individuals in families affected by HD differ depending on whether they are affected, gene-positive, at risk and/or carers. Those diagnosed with the disease may require a neurologist, psychiatrist, Huntington's disease specialist nurse (HD nurse), speech and language therapist, occupational therapist, dietician, physiotherapist, general practitioner (GP), Huntington's Disease Association (HDA) social worker, researchers and community support services. Those that are gene-positive may require genetic services, psychiatrist, neurologist, HD nurse, HDA and GP. Those at risk may require genetic services, psychiatrist, HD Nurse, neurologist, HDA, and GP. Carers may require HD Nurse, HDA, social worker, respite care facilities and GP. Caring for a family is therefore complex and the needs of these individuals need to be identified and interactions between professionals and family co-ordinated. We have established a model in which the HD nurse provides much of the assessment and co-ordination of care for these individuals.

\section{THE YKL-40 PROTEIN IS NOT ELEVATED IN CEREBROSPINAL FLUID FROM HUNTINGTON'S DISEASE PATIENTS}

Tua Vinther-Jensen, Esben Budtz-Jørgensen, Anja H. Simonsen, Kristian Winge, Lena E. Hjermind, Jørgen E. Nielsen

\section{Abstract.}

Introduction: Neuroinflammation is a well-known phenomenon in Huntington's disease (HD). The inflammation is considered to be specific, mediated by the immune cells of the brain, as there are no indications of influx of peripheral immune cells to the brain. The inflammation is probably an early event in the pathological processes given that immune activation is shown to be present up till 15 years before expected disease onset. But how and why the inflammation process is initiated and to which extent the different factors are involved is poorly understood. The YKL-40 protein is a member of the glycosyl hydrolase family 18. It is expressed and released during cellular differentiation in different cells, and up-regulated in inflamed tissues in a variety of inflammatory diseases. YKL-40 is significantly elevated in different acute and chronic neuroinflammatory diseases; however, the exact function is not known but involvement in migration of astrocytes and microglia cells have been reported. Objectives and Methods: We quantified the YKL-40 protein levels in Cerebrospinal fluid (CSF) and plasma from 30 
manifest HD patients, 30 premanifest HD gene-expansion carriers, and 15 healthy controls by ELISA technique to explore its potential as a biomarker of inflammation in HD and in the longer term to investigate whether YKL-40 is part of, or affected by the HD pathogenesis.

Results and Conclusion: An age dependent elevation of the YKL-40 protein, as reported in studies of other neurodegenerative disorders was measured in all three groups. There was no significant difference in the concentration of the YKL-40 protein in CSF between the healthy controls, the premanifest HD gene-expansion carriers and HD patients. However, there was a trend towards a higher YKL-40 CSF concentration in manifest $\mathrm{HD}$ patients compared to premanifest HD gene-expansion carriers and healthy controls. We conclude that the YKL-40 protein does not seem to be a useful marker of neuroinflammation in HD and the elevation of YKL-40 concentration appears to be a late event in HD pathogenesis, presumably reflecting an unspecific reaction to neurodegeneration.

\section{TRANSCRANIAL BRAIN SONOGRAPHY FINDINGS IN HUNTINGTON'S DISEASE PATIENTS AND HEALTHY INDIVIDUALS}

Rita de Cássia Leite Fernandes, Ana Lucia Zuma de Rosso, Maurice Borges Vincent, Nordeval Cavalcante Araújo

\footnotetext{
Abstract.

Introduction: Transcranial sonography (TCS) of the brain parenchyma is a promising neuroimaging technique for investigating movement disorders. Hyperechogenicity of the substantia nigra (SN) was found to enable the diagnosis of Parkinson's disease with high predictive values. Nevertheless, few studies evaluated the diagnostic role of TCS in Huntington's disease (HD). The aim of this study was to compare TCS findings in HD patients and healthy controls.

Method: TCS was performed at the temporal acoustic window with a $1.3-2.5 \mathrm{MHz}$ phased array probe (Acuson $\mathrm{X} 300$, Siemens) following an international investigation protocol. ${ }^{1}$ The HD patients $(n=4)$ were recruited in an outpatient movement disorders clinic and controls were recruited from among caregivers $(n=60)$. The SN echogenic area was encircled at the mesencephalic axial plane. At the basal ganglia plane, echogenicity of the lentiform nucleus (LN) was evaluated and the transversal diameters of the 3rd ventricle and of the frontal horns of the lateral ventricles were measured. SPSS 17.0 was used for data analysis computed as the mean \pm SD. The Mann-Whitney test was employed for group comparisons.

Results: The study groups were age-matched (controls: $57.3 \pm 15.8$ years old and HD: $50.7 \pm 18.2$ years old). HD patients had a mean disease duration of $111.0 \pm 47.4$ months (range: $72-180$ months). The mean diameter of the 3rd ventricle was larger in the HD group (controls: $4.3 \pm 1.8 \mathrm{~mm}$, HD: $6.7 \pm 2.9 \mathrm{~mm}, p=0.032$ ) likewise that of the frontal poles (controls: $15.1 \pm 2.7 \mathrm{~mm}$, HD: $21.0 \pm 1.4 \mathrm{~mm}, p=0.004$ ). A polygonal shape of the frontal poles could be depicted in all HD patients in contrast to the comma shape of normal individuals (Figure). SN hyperechogenic area and LN echogenicity were similar between the groups.
}

Conclusions: TCS performed on HD patients in a specialized ambulatory setting found out significant differences between patients and controls, albeit the small number of patients in the sample. The higher ventricular diameters measurements may reflect the cerebral atrophy along with the disease, and the different shape contour observed in the frontal poles might point to its distinctive caudate nucleus atrophy. This study suggests that TCS, a harmless and non-expensive technique, might be useful in improving diagnosis and follow-up of HD patients and deserves further investigation. ${ }^{1}$ Walter et al. Ultrasound in Med\&Biol 2007;33:15-25

\section{TWENTY FOUR CYCLES OF PREIMPLANTATION GENETIC DIAGNOSIS EXCLUSION TESTING FOR HUNTINGTON DISEASE. SHOULD WE PAUSE FOR THOUGHT?}

\section{Alison Lashwood, Caroline Ogilvie}

Abstract. Preimplantation genetic diagnosis (PGD) offers an alternative reproductive option to prenatal diagnosis. Huntington disease (HD) exclusion testing of embryos is available at our PGD Centre for couples where one partner has a parent affected by HD, but who decides against knowing their own HD status. Fifteen couples underwent 24 fresh cycles of PGD and 4 frozen embryo replacement cycles at our centre using haplotype analysis to exclude embryos carrying a chromosome 4 from the affected grandparent. Of the "at risk" partners, 7 were male and 8 female. These cycles resulted in a $53 \%$ clinical pregnancy rate per couple followed by the birth of eight singletons and 2 sets of twins. Most couples had normal fertility. In total, 168 embryos were biopsied (mean 7 embryos /cycle), and 143 were clearly diagnosed as high or low risk. Sixty five $(45 \%)$ were discarded as they carried the high risk grandparental chromosome 4 . In 4 cycles no embryos were available for transfer. These discarded embryos may have been free of the HD mutation, and may have represented a couple's only chance of pregnancy. There are well documented complex reasons why individuals at risk of HD decline testing. PGD is an expensive procedure, the cost of which is born either by the state or by patients directly, and is associated with female health risks. In addition, babies born following PGD have an increased risk of neonatal complications, congenital malformations and lower birth-weight compared with spontaneously conceived infants. These considerations, in conjunction with our data, indicate that the benefits of PGD for HD by exclusion testing require careful evaluation.

\section{USE GOAL ATTAINMENT SCALING (GAS) AS A TOOL TO EVALUATE THE PROCESSES OF REHABILITATION FOR PEOPLE WITH HUNTINGTON'S DISEASE}

Valentina Aviles, Francisco Fuentealba, Natalia Rojas, Carolina Silva, Sara Tapia, Daniela Alburquerque, Pedro Chana-Cuevas

Abstract. It evaluated the rehabilitation program by Goal attainment scaling (GAS) which allows the standardization of 
results in the rehabilitation process for patients who received a transdisciplinary approach in rehabilitation.

Method: GAS is a measurement method that accommodates multiple individual patient goals, and has a scoring system that allows comparisons between patients. The formulation of objectives is under the concepts of the acronym SMART (specific, measurable, achievable, realistic / relevant and timed) were evaluated initially set targets centered rehabilitation and functional treatment goals, set individually and participatory. The project was developed over a period of 180 days. In an open study 22 patients were evaluated ( 9 men and 13 women) with Huntington's disease 6 years of evolution. With an average of $4.4 \pm 1.2$ per patient goals.

Results: are expressed with average and ranges. $\times$ plus or minus standard deviation. Results: Compliance is achieved the objectives of $90.7 \pm 14.6 \%$. the score obtained by GAS scale at baseline was $35.1 \pm 1.3$ and the score at the end of the intervention was $65.68100108 \pm 8.7$. With a $p<0.001$.

Conclusion: In an open experience describes the objectives (SMART), the results assessed by GAS scale, which appears as an excellent alternative to evaluate interventions with varied objectives.

\section{VOLTAGE-GATED CALCIUM CHANNEL BLOCKERS AND REVERSIBLE NMDA RECEPTOR ANTAGONISTS AS NEUROPROTECTIVE TOOLS IN HUNTINGTON'S DISEASE}

Flavia Rodrigues Silva, Andressa Pereira Mouro, Luciene Bruno Vieira, Fabiola Mara Ribeiro

\footnotetext{
Abstract.

Objective: Investigating the pharmacological and biochemical mechanisms that involve voltage-gated $\mathrm{Ca} 2+$ channels (VGCC) blockers and reversible antagonist of NMDA receptor in Huntington's Disease (HD).

Introduction: Huntington's Disease (HD) is an autosomal dominant neurodegenerative disorder characterized by symptoms of involuntary body movement, loss of cognitive function, psychiatric disorder, inevitably leading to death. The huntingtin $(\mathrm{Htt})$ mutated protein exhibiting a polyglutamine expansion in the amino terminal region, is considered to be the cause of $\mathrm{DH}$ and patients with the mutated form of this protein develop the disease. $\mathrm{Ca} 2+$ signaling alterations are closely linked to neuronal death processes, which can be associated with an excitotoxic increase in cytosolic $\mathrm{Ca} 2+$ levels in $\mathrm{HD}$. voltage-gated $\mathrm{Ca} 2+$ channels L-type (L-VGCC) and the N type (N-VGCC) may disturb $\mathrm{Ca} 2+$ homeostasis by increasing the release of glutamate from the presynaptic terminals. Several studies indicate that venom toxins from Phoneutria nigriventer presents neuroprotective effects and demonstrate that these toxins exhibit good tolerance.

Methods: Through experiments Neuronal primary, Cell Death Assay, Immunoblotting and Co-immunoprecipitation, We have selected four VGCC blockers, PhTx3-1, PhTx3-4, Isradipine and Nifedipine, and a toxin that is a reversible antagonist of NMDA receptor, PhTx4-4-5, and tested at lower and right concentrations whether these drugs were able to protect against neuronal death induced by glutamate. In
}

addition to that, expression of these channels and it's interaction with $\mathrm{Htt}$ in the cortex, striatum and hipocampus of a HD mouse model, BACHD, was also observed.

Results: Our results demonstrate that Nifedipine $(0.1 \mathrm{nM})$, PhTx4-4-5 (0.001 nM to $4 \mathrm{nM}$ ), PhTx3-1 (1 nM) and Isradipin $(1 \mathrm{nM})$ promote neuroprotection against death induced by glutamate as compared to control group. Furthermore, we observed that L-VGCC is more expressive in cortex and hippocampus at seven old mice. In other hand, N-VGCC has reduced it's expressiion as the age increases in HD.

Conclusion: These results demonstrate that VGCCs blockers might have the potential to protect against neuronal death that occours in HD and a possible involvement of L-Type and $\mathrm{N}$-Type VGCC with mHtt protein on symptomatic phase of the disease. Thus, these VGCCs as reversible antagonist of NMDA receptor may be potential therapeutics for the treatment of HD and other neurodegenerative diseases.

\section{WHAT DO WE KNOW ABOUT HUNTINGTIN FUNCTION IN HD?}

\section{Elena}

Abstract. Huntingtin (htt) is the $\sim 800$ million-year old protein product of the Huntington's disease (HD) gene. The gene contains a polymorphic tri-nucleotide $\mathrm{CAG}$ repeat that is translated into polyglutamine amino acid (polyQ) residues in the protein. When this polyQ stretch at the 18 aminoacid (aa) position of the protein expands to over 39 residues, HD occurs, a fatal, genetically dominant, neurodegenerative disease. The CAG repeats are well conserved in deuterostomes, which suggests that they are an ancestral feature retained during the evolution of the protein. Htt carries a number of specific activities in the adult brain; for instance, it promotes transcription of neuronal genes among which is the BDNF, a neurotrophin critical for the survival and activity of cortical and striatal neurons that degenerate in HD. This presentation will highlight the power of combining evolutionary and developmental approaches to the study of the biology of disease-genes and will review the more recent discoveries of the function of htt in the developing and mature brain.

\section{WISHES FOR THE END OF LIFE IN HUNTINGTON'S DISEASE}

Suzanne J Booij, Aad Tibben, Raymund AC Roos, Dick P Engberts

\section{Abstract.}

Introduction: In the Netherlands euthanasia or physician assisted suicide (PAS) is legal under strict conditions after a direct request from a patient or based on an advance directive. Each year approximately 7 requests for euthanasia from a HD patient are granted. There seemed to be an increase in conversations about wishes for the end of life between physicians and patients, but this was never investigated. This is the first study to investigate the end-of-life wishes of HD patients.

Objective: To investigate presence and content of end-of-life wishes amongst patients with HD and to assess relationship 
with demographic or disease specific characteristics.

Methods: Based on preceding qualitative research a custommade questionnaire was developed. 297 Dutch patients registered in the Leiden HD Registry Database were screened, 55 excluded because of declined informed consent, severe depression, suicidal ideation or the lack of accurate and up to date medical information. 242 questionnaires were sent out in 2011. Information on demographic variables was collected together with clinical characteristics such as TFC, MMSE, UHDRS-M. Presence of wishes was correlated with demographic and clinical characteristics.

Results: A total of 134 patients $(55.4 \%)$ returned the questionnaire. One-hundred-and-one respondents (75\%) reported to have some kind of thoughts for the end of life. For 15 respondents $(11 \%)$ these thoughts concerned care; 86 respondents $(64 \%)$ reported to also have thoughts about euthanasia or physician assisted suicide (PAS). The presence of thoughts about the end of life was related to being familiar with HD in the family but not to any other socio-demographic or clinical variable.

Conclusion: Thoughts about and wishes for the end of life are widely present amongst patients with HD and known gene carriers and these thoughts or wishes concern euthanasia or PAS in a majority of the respondents. Knowing if end-of-life wishes are indeed increasingly present and knowing the content of these wishes could be helpful for physicians. It could help treatment and guidance of HD patients, especially in light of the absence of any effective treatment. Based on this study it seems impossible to determine which patients will have thoughts or wishes for the end of life and which patients do not. Therefore these questions should be addressed in every patient with HD.

\section{"IT'S BETTER TO KNOW THAN NOT KNOW"- FIRST HAND ACCOUNTS OF PREDICTIVE GENETIC TESTING IN ADOLESCENTS FOR ADULT-ONSET CONDITIONS}

\author{
Cara Mand, Rony Duncan, Lynn Gillam, \\ Martin Delatycki
}

\begin{abstract}
.
Background: Asymptomatic individuals at risk of genetic conditions can undergo predictive testing (PT) to determine if they will develop the condition later in life. PT is routinely offered to adults at risk of late-onset conditions (e.g. Huntington disease). More controversial is PT in minors for adult-onset conditions where no effective treatment or prevention exists. The ethical discourse concerning PT in minors is lively, yet it remains one of speculation, with a relative scarcity of empirical evidence.
\end{abstract}

Aim: To explore young people's experience of being tested for an adult-onset condition for which no medical intervention exists.

Methods: Semi-structured interviews with nine young people who underwent PT for adult-onset conditions as minors (6 gene-positive, 3 gene-negative). Interviews were transcribed and thematically analysed.

Results: Interviews revealed a range of harms and benefits associated with the PT experience. Harms were not limited to individuals who received a gene-positive result. Participants identified potential harms with being refused a test as well as the testing process; however these were outweighed by the benefits that arose. Benefits were described by all participants, regardless of result. Participants experienced difficultly living with uncertainty prior to testing, and all described feeling relieved when receiving their test result. Irrespective of the result, no participant regretted being tested.

Conclusions: These data add to the breadth and complexity of the discourse about PT in minors, highlighting factors which have been previously under-represented in the literature. Current approaches towards counselling and other processes prior to PT may need to be re-considered in light of the negative experiences of the testing process reported by some participants. For the first time this study gives voice to the adolescents who are the centre of this debate, inviting reconsideration of whether the current guidelines have reached the correct balance in terms of pros and cons of PT in minors.

\section{"PREQUEL:" TRIAL OF COENZYME Q10 IN PREMANIFEST HD: FEASIBILITY OF FUTURE TRIALS}

Kevin M. Biglan, Annie Killoran, M. Flint Beal, Wayne Matson, Elaine Julian-Baros, Shan Gao, Michael P. McDermott, HSG PREQUEL Investigators

\section{Abstract.}

Background: PREQUEL is the first multi-center interventional trial in pre-manifest Huntington disease (HD). Objective: To assess the safety and tolerability of coenzyme Q10 (CoQ) in subjects with pre-manifest HD, and the feasibility of conducting therapeutic trials in this population.

Methods: PREQUEL is a phase II randomized, double blind, multi-center trial of $600 \mathrm{mg}, 1200 \mathrm{mg}$ and $2400 \mathrm{mg}$ per day of CoQ. The 90 study participants were expansion positive but pre-manifest (diagnostic confidence score of $<3$ on the UHDRS). Tolerability was defined as completion of the 20week study on the originally randomized treatment assignment, with a pre-specified threshold of $75 \%$.

Results: The mean subject age was 39.5 , with $47 \%$ male, and $85 \%$ employed. The mean total UHDRS motor score was 3.5 and the mean estimated time to HD onset (Langbehn formula) was $11.5+/-11.1$ years. PREQUEL had $93 \%$ retention and all groups exceeded the pre-defined $75 \%$ tolerability threshold. The incidence of adverse events $(45 \%, 50 \%$ and $63 \%$, respectively) was not significantly different among the CoQ dosages. CoQ levels increased in all dosage groups, and there was no significant difference among the three groups: Mean +/- SD baseline levels were (in order of CoQ dosage) 0.77 $+/-0.30,0.74+/-0.34$, and $0.98+/-1.22 \mathrm{mcg} / \mathrm{ml}$, respectively, and 20 -week levels were $2.55+/-1.82,2.72+/$ 1.76 , and $3.22+/-1.72$. Recruitment at the 10 initial sites started rapidly but plateaued, so the number of in-person visits was reduced, and three sites were added. There was no significant change in $8 \mathrm{OHdG}$ levels at 20 weeks for any dosage. There was no relationship between $80 \mathrm{HdG}$ level at baseline and the predicted time to onset.

Conclusion: CoQ was well tolerated at dosages up to 2400 $\mathrm{mg} /$ day for 20 weeks in subjects with pre-manifest HD. The study shows the feasibility of conducting clinical trials in this population. $8 \mathrm{OHdG}$ did not perform well as biomarker. 
Finally, power analysis indicated that if PREQUEL is Designs involving preselection of subjects based on CAP representative of premanifest HD subjects who would enroll score or predicted years to onset should be considered for in a therapeutic trial, such trials, would require a large future therapeutic trials. numbers of subjects to detect differences in motor onset. 


\section{Author Index}

Abler, B., see Weydt, P., 36

Albuquerque, M.V.C., see de Souza, P.V.S., 39

Alburquerque, D., see Aviles, V., 59

Alburquerque, D., see Rey, V., 30

Allain, P., 31

Amico, E., see Pardo, A.D., 51

Andrew, S., see Wedderburn, S., 50

Añez, E.S., 39

Antonopoulos, S., see Goh, A., 27

Araújo, N.C., see de Cássia Leite Fernandes, R., 59

Armeso, D., see Mariscal, N., 33

Armesto, D., see Cubo, E., 58

Arroyo, M.A.R., see Cubo, E., 51

Aviles, V., 59

Aylward, E.H., 21

Azevedo, L.R., see Spedo, C.T., 24

Baker, K., see Stout, J.C., 56

Barbosa, E.R., see Capato, T., 34, 48

Barker, R., see Pakpoor, J., 19

Barreira, A.A., see Spedo, C.T., 24

Barsottini, O., see de Castilhos, R.M., 38

Barsottini, O., see Jardim, L.B., 38

Barsottini, O.G.P., see de Souza, P.V.S., 39

Barth, K., see Handley, O., 35

Bassan, M., see Darpo, B., 26

Bassan, M., see Landwehrmeyer, B., 30

Bassan, M., see Rabinovich-Guilatt, L., 15, 49

Bates, G., see McCourt, A.C., 36

Bates, G., see Rattray, I., 20

Bates, G.P., see Sathasivam, K., 17

Beal, M.F., see Biglan, K.M., 61

Begeti, F., see Pakpoor, J., 19

Beilby, J., see Wedderburn, S., 50

Bek, J., 21

Bel, E.A.D., see dos Santos, B.L., 37

Belz, H., see Süssmuth, S.D., 26

Benaich, S., see Gargiulo, M., 32

Benavides, O., see Rey, V., 30

Benjamin, A.C., see Sathasivam, K., 17

Berenguer, J., see Hennig, B.L., 25

Bezerra, J.M.F., see Spitz, M., 28

Biglan, K.M., 61
Björkqvist, M., see McCourt, A.C., 36

Böhme, R., see Nickchen, K., 52

Bondulich, M.K., see Sathasivam, K., 17

Bonelli, R., see van Duijn, E., 45

Booij, S.J., 60

Bopsin, P.S., see de Castilhos, R.M., 38

Bornstedt, A., 44

Borowsky, B., see Domínguez, D.J.F., 28

Borowsky, B., see Poudel, G.R., 35

Borowsky, B., see Stout, J.C., 56

Bourke, D., see Roxburgh, R., 48

Bouwens, J.A., see Hubers, A.A.M., 19

Bradaia, A., see Mielcarek, M., 36

Bruno, R., see Rabinovich-Guilatt, L., 49

Budtz-Jørgensen, E., see Petersen, M., 43

Budtz-Jørgensen, E., see Vinther-Jensen, T., 58

Bullock, J., see Schut, M., 21

Burgunder, J.-M., see Handley, O., 35

Busse, M., see Devlin, R., 55

Butler, R., see Mielcarek, M., 36

Cachorro, A.M., see Posadas, J.J.R., 24, 31

Caetano, G., 29, 47

Caetano, G., see Júlio, F., 57

Caillaud, M., see Allain, P., 31

Calero, V.L.G., 43

Callaghan, J., see van Duijn, E., 45

Caltagirone, C., see Paola, M.D., 32

Caltagirone, C., see Phillips, O., 41

Camara, R., see Cubo, E., 58

Cámara, R.J.A., see Posadas, J.J.R., 24, 31

Campolongo, A., see Martinez-Horta, S., 45

Campolongo, A., see Perez, J.P., 51

Capato, T., 34, 48

Castelo-Branco, M., see Caetano, G., 47

Castelo-Branco, M., see Júlio, F., 57

Castilhos, R.M., see Jardim, L.B., 38

Cattaneo, E., 37

Chana-Cuevas, P., see Aviles, V., 59

Chana-Cuevas, P., see Rey, V., 30

Chavez, C., see Añez, E.S., 39

Chehere, P., 37

Chern, L.H., see Mestre, T., 23 
Chestkov, I.V., see Lagarkova, M.A., 16 Cheung, M., see Dysart, J., 58

Chiu, E., see Goh, A., 27

Christie, D., see Waldvogel, H., 46

Chua, P., see Domínguez, D.J.F., 28, 50

Chua, P., see Poudel, G.R., 22, 35

Churchyard, A., see Domínguez, D.J.F., 28, 50

Churchyard, A., see Labuschagne, I., 40

Churchyard, A., see Poudel, G.R., 22, 35

Cobbaert, C.M., see Hubers, A.A.M., 19

Cole, J.H., see Hobbs, N.Z., 54

Conde, R.M., see Spedo, C.T., 24

Connolly, C., see Leavitt, B.R., 42

Conrad, A.L., see Lee, J.K., 57

Cook, C., see Devlin, R., 55

Coppi, A.A., see Ladd, F.V.L., 31

Cowlishaw, S., see Stout, J.C., 56

Cox, K., see Goh, A.M., 48

Craufurd, D., see Bek, J., 21

Craufurd, D., see van Duijn, E., 45

Crum, W.R., see Rattray, I., 20

Cubo, E., 51, 58

Cunha, G., see Caetano, G., 29

D’Ávila, R., see Saute, J.A.M., 52

da Costa Valadares, M.V., see Spedo, C.T., 24

da Silva, A.L., see de Castilhos, R.M., 38

da Silva, A.L., see Jardim, L.B., 38

Dac, V., see Spedo, C.T., 24

Darpo, B., 26

Davila, G.O.O., see Calero, V.L.G., 43

Davila, G.O.O., see Quiñones, H.J.C., 41

Dawson, J. see Nopoulos, P., 17

Dawson, J.D., see Lee, J.K., 57

Dayan, M., 18

de Aragão, B.C., 44

de Aragão, B.C., see Valadão, P.A.C., 19

de Bobadilla, R.F., see Martinez-Horta, S., 45

de Bobadilla, R.F., see Perez, J.P., 51

de Carvalho, T.G., see de Aragão, B.C., 44

de Cássia Leite Fernandes, R., 59

de Castilhos, R.M., 38

de Castilhos, R.M., see Saute, J.A.M., 52

de Castilhos, R.M., see Souza, A.F.D., 53

de Freitas, I.C., see Spedo, C.T., 24

de Jesus, M.S., see Spedo, C.T., 24

de Mello Rieder, C.R., see Souza, A.F.D., 53

de Rezende Pinto, W.B.V., see de Souza, P.V.S., 39

de Rosso, A.L.Z., see de Cássia Leite Fernandes, R., 59

de Souza Alencar, M.A.S., 30 de Souza, P.V.S., 39

De, D.O.G., see Saute, J.A.M., 52

del Mar Amador, M., see Nickchen, K., 52

Delatycki, M., see Mand, C., 61

Delgado, E.C., see Mariscal, N., 33

Delgado, E.C., see Posadas, J.J.R., 24

Delgado, E.C., see Posadas, J.J.R., 31

Delgado, W., see Añez, E.S., 39

den Dunnen, J., see Schut, M., 21

Descallas, A.M., see Cubo, E., 51

Descalls, A.M., see Cubo, E., 58

Descalls, A.M., see Mariscal, N., 33

Descalls, A.M., see Posadas, J.J.R., 24, 31

Deserno, L., see Nickchen, K., 52

Detloff, P.J., see Sathasivam, K., 17

Devine, J., see Dysart, J., 58

Devlin, R., 55

Domínguez, D.J.F., 28, 50

Domínguez, D.J.F., see Poudel, G.R., 22, 35

Donis, K.C., see de Castilhos, R.M., 38

Donis, K.C., see Jardim, L.B., 38

Donis, K.C., see Saute, J.A.M., 52

dos Santos, B.L., 37

du Montcel, S.T., see Gargiulo, M., 32

Dumas, E., see Hart, E., 28

Duncan, R., see Mand, C., 61

Durr, A., see Gargiulo, M., 32

Durr, A., see Hobbs, N.Z., 54

Dysart, J., 58

Dysart, J., see Roxburgh, R., 48

Egan, G.F., see Domínguez, D.J.F., 28, 50

Egan, G.F., see Poudel, G.R., 22, 35

Eimeren, T.V., see Mestre, T., 23

el Haj, M., see Allain, P., 31

Elben, S., see Vesper, J., 17

Elena, 60

Elian see Jardim, L.B., 38

Elifani, F., see Paola, M.D., 32

Elifani, F., see Phillips, O., 41

Engberts, D.P., see Booij, S.J., 60

Epping, E., see Nopoulos, P., 17, 29

Epping, E.A., see Lee, J.K., 57

Etcheverry, J.L., see Parisi, V., 42

Eyal, E., see Landwehrmeyer, B., 30

Farmer, R., see Wild, E., 45

Farmer, R.E., see Hobbs, N.Z., 54

Faull, R., see Dysart, J., 58

Faull, R., see Mehrabi, N., 27

Faull, R., see Schut, M., 21 
Faull, R., see Singh-Bains, M., 56

Faull, R., see Waldvogel, H., 46

Faull, R.L., see Sathasivam, K., 17

Favellato, M., see Pardo, A.D., 51

Fedotova, E.Y., see Lagarkova, M.A., 16

Feingold, J., see Gargiulo, M., 32

Ferguson, S.S.G., see Guimarães, I.M., 40

Ferrea, S., see Vesper, J., 17

Ferrer, O., see Añez, E.S., 39

Finol, Z., see Añez, E.S., 39

Finucane, G., see Dysart, J., 58

Fisch, D.F., see Wild, E., 45

Fischer, W., see Gelderblom, H., 18

Fiszer, A., see Krzyzosiak, W.J., 53

Folmann, L.B., see Nielsen, S.M.B., 34

Formoso, D.A., see Posadas, J.J.R., 24, 31

Foss, M.P., see Spedo, C.T., 346

Franklin, S.A., see Mielcarek, M., 36

Frich, J.C. see van Walsem, M., 15

Frich, J.C., see Piira, A., 16

Frost, C., see Hobbs, N.Z., 54

Fuentealba, F., see Aviles, V., 59

Fullam, R., see Handley, O., 35

Furtado, G.V., see de Castilhos, R.M., 38

Furtado, G.V., see Jardim, L.B., 38

Furtado, G.V., see Saute, J.A.M., 52

Furtado, G.V., see Souza, A.F.D., 53

Gaino, S.B., see Spedo, C.T., 24

Gale, R., see Rattray, I., 20

Galka-Marciniak, P., see Krzyzosiak, W.J., 53

Gao, S., see Biglan, K.M., 61

Garcias, R., see Valadão, P.A.C., 19

Gargiulo, M., 32

Gatto, E., see Parisi, V., 42

Gelderblom, H., 18

Georgiou-Karistianis, N., see Domínguez, D.J.F., 28, 50

Georgiou-Karistianis, N., see Labuschagne, I., 40

Georgiou-Karistianis, N., see Poudel, G.R., 22, 35

Gheno, T.C., see de Castilhos, R.M., 38

Gheno, T.C., see Jardim, L.B., 38

Gheno, T.C., see Saute, J.A.M., 52

Gheno, T.C., see Souza, A.F.D., 53

Gil, C., see Cubo, E., 58

Gillam, L., see Mand, C., 61

Giltay, E., see Hart, E., 28

Giltay, E.J., see Hubers, A.A.M., 19

Giltay, E.J., see van Duijn, E., 45

Gipson, T.A., see Sathasivam, K., 17

Gisske, K., see Nickchen, K., 52

Giuliano, J., see Mestre, T.A., 34
Godeiro-Junior, C., see de Castilhos, R.M., 38

Godeiro-Junior, C., see Jardim, L.B., 38

Goh, A., 27

Goh, A.M., 48

Goldblatt, J., see Wedderburn, S., 50

Gomes, M.P.S.M., see Valadão, P.A.C., 19

González, L., see Añez, E.S., 39

Gordon, R., see Mantovani, S., 23

Gray, M.A., see Domínguez D.J.F., 28

Gray, M.A., see Domínguez D.J.F., 50

Gray, M.A., see Poudel, G.R., 35

Grivennikov, I.A., see Lagarkova, M.A., 16

Groiss, S.J., see Vesper, J., 17

Grön, G., see Weydt, P., 36

Grubb, E., 24

Grueninger, S., see Wild, E., 45

Guatimosim, C., see de Aragão, B.C., 44

Guatimosim, C., see Valadão, P.A.C., 19

Guimarães, I.M., 40

Gunn, D., see McMillan, J., 25

Haddad, M., see Capato, T., 34, 48

Haider, S., see Hobbs, N.Z., 54

Haider, S., see Wild, E., 45

Handley, O., 35

Handley, O.J., see Mestre, T.A., 34

Hannan, A., see Goh, A.M., 48

Hart, E., 28

Hasholt, L., see Nielsen, S.M.B., 34

Hayardeny, L., see Leavitt, B.R., 42

Hayden, M.R., see Leavitt, B.R., 42

Hayen, A., see McMillan, J., 25

Hayen, A., see Mito, R., 41

Heemskerk, A.-W., 33, 56

Heidari, R., 46

Heijnen, B., see Heemskerk, A.-W., 56

Hennig, B.L., 25, 55

Hensman, D., see Wild, E., 45

Herbert J., see Calero, V.L.G., 43

Hermant, E., see Pihet, V., 23

Herson, A., see Gargiulo, M., 32

Hjermind, L.E., see Vinther-Jensen, T., 58

Hobbs, N.Z., 54

Hobbs, N.Z., see Aylward, E.H., 21

Hogg, V., see Mehrabi, N., 27

Hogg, V., see Singh-Bains, M., 56

Housman, D.E., see Sathasivam, K., 17

Howland, D., see Sathasivam, K., 17

Hubers, A.A.M., 19

Hubers, A.A.M., see van Duijn, E., 45

Hulter, B., 53 
Illarioshkin, S.N., see Lagarkova, M.A., 16 Indra, S.M., see Cubo, E., 51

Inuabasi, L., see Mielcarek, M., 36

Januário, C., see Caetano, G., 29, 47

Januário, C., see Júlio, F., 57

Jardim, L.B., 38

Jardim, L.B., see de Souza, P.V.S., 39

Jardim, L.B., see Souza, A.F.D., 53

Jauffret, C., see Gargiulo, M., 32

Josefsen, K., see Nielsen, S.M.B., 34

Julian-Baros, E., see Biglan, K.M., 61

Júlio, F., 57

Júlio, F., see Caetano, G., 29, 47

Jutras, M.-F., see Gargiulo, M., 32

Kampinga, H.H., 32

Kaplan, R.F., see Hennig, B.L., 25

Kassubek, J., see Bornstedt, A., 44

Keenan, K.F., 37

Kieburtz, K., see Landwehrmeyer, B., 30

Killoran, A., see Biglan, K.M., 61

Kim, E., see Schut, M., 21

Kim, E., see Waldvogel, H., 46

Kiselev, S.L., see Lagarkova, M.A., 16

Klyushnikov, S.A., see Lagarkova, M.A., 16

Knutsen, S.F., see van Walsem, M., 15

Knutsen, S.F., see Piira, A., 16

Kordaschia, C., see Labuschagne, I., 40

Krzyzosiak, W.J., 53

Kulisevsky, J., see Martinez-Horta, S., 45

Kulisevsky, J., see Perez, J.P., 51

Kumar, V., see Mantovani, S., 23

Labuschagne, I., 40

Ladd, A.A.B.L., see Ladd, F.V.L., 31

Ladd, F.V.L., 31

Lagarkova, M.A., 16

Lage, M., see Grubb, E., 24

Lahiri, N., see Wild, E., 45

Land, V., see Quiñones, H.J.C., 41

Landles, C., see Mielcarek, M., 36

Landles, C., see Sathasivam, K., 17

Landles, C., see Wild, E., 45

Landwehrmeyer, B., 30

Landwehrmeyer, B., see Gelderblom, H., 18

Landwehrmeyer, B., see Handley, O., 35

Landwehrmeyer, B., see Hobbs, N.Z., 54

Landwehrmeyer, B., see Mestre, T.A., 34

Landwehrmeyer, G.B., see Süssmuth, S.D., 26

Landwehrmeyer, G.B., see Weydt, P., 36
Langdon, R., see McMillan, J., 25

Lashwood, A., 59

Lautenschlager, N., see Goh, A.M., 48

Leavitt, B.R., 42

Lebedeva, O.S., see Lagarkova, M.A., 16

Lee, J., see Nopoulos, P., 17, 29

Lee, J., see Wedderburn, S., 50

Lee, J.K., 57

Lehmensiek, V., see Süssmuth, S.D., 26

Li, R., see Mantovani, S., 23

Liebeck, T., see Wedderburn, S., 50

Lima, M.-A.F.D., see de Castilhos, R.M., 38

Lima, M.-A.F.D, see Jardim, L.B., 38

Lindenberg, K.S., see Bornstedt, A., 44

Loi, S., see Goh, A., 27

Lorca, P., see Rey, V., 30

Lowe, M., see Waldvogel, H., 46

Loy, C., see McMillan, J., 25

Loy, C., see Mito, R., 41

Lukasiewicz-Hagai, E., see Darpo, B., 26

Lukasiewicz-Hagai, E., see Landwehrmeyer, B., 30

Lukasiewicz-Hagai, E., see Rabinovich-Guilatt, L., 49

Macdonald, D., see Wild, E., 45

Maglione, V., see Paola, M.D., 32

Maglione, V., see Pardo, A.D., 51

Maglione, V., see Phillips, O., 41

Magnotta, V., see Nopoulos, P., 17

Maio, L.D., see Michele, G.D., 39

Malejko, K., see Weydt, P., 36

Mand, C., 61

Mantovani, S., 23

Marchand, M. see Rabinovich-Guilatt, L., 49

Marcinkowski, J.T., see Zielonka, D., 49

Marinus, H., see Heemskerk, A.-W., 56

Mariscal, N., 33

Marks, P.A., see Mielcarek, M., 36

Marques, V.D., see Spedo, C.T., 346

Martinez, M.A.M.I., see Calero, V.L.G., 43

Martinez, M.A.M.I., see Quiñones, H.J.C., 41

Martinez-Horta, S., 45

Martinez-Horta, S., see Perez, J.P., 51

Massarelli, M., see Michele, G.D., 39

Mateos, A., see Cubo, E., 58

Mathews, K., see Lee, J.K., 57

Mathews, K., see Nopoulos, P., 17

Matson, W., see Biglan, K.M., 61

McCourt, A.C., 36

McCusker, E., see McMillan, J., 25

McCusker, E., see Mito, R., 41

McDermott, M.P., see Biglan, K.M., 61 
McGrath, F., see Wedderburn, S., 50

McHugh, M., see Nopoulos, P., 29

McKee, L., see Keenan, K.F., 37

McLean, T., see Gelderblom, H., 18

McLean, T., see Handley, O., 35

McMillan, J., 25

McMillan, J., see Mito, R., 41

Mehrabi, N., 27

Merdjan, H., see Rabinovich-Guilatt, L., 49

Mestre, T., 23

Mestre, T.A., 34

Michele, G.D., 39

Middelkoop, H., see Hart, E., 28

Miedzybrodzka, Z., see Keenan, K.F., 37

Mielcarek, M., 36

Mikalsen, G., see Piira, A., 16

Mikalsen, G., see van Walsem, M., 15

Mills, E.D., see Lee, J.K., 57

Mito, R., 41

Modo, M., see Rattray, I., 20

Monte, T.L., see Saute, J.A.M., 52

Moreira, A.L., see Ladd, F.V.L., 31

Mouro, A.P., see Silva, F.R., 60

Mueller, H.-P., see Hobbs, N.Z., 54

Müller, H.-P., see Bornstedt, A., 44

Mykowska, A., see Krzyzosiak, W.J., 53

Neto, G.S.S., see Spedo, C.T., 24

Neueder, A., see Sathasivam, K., 17

Nickchen, K., 52

Nielsen, J.E., see Petersen, M., 43

Nielsen, J.E., see Vinther-Jensen, T., 58

Nielsen, S.M.B., 34

Nielsen, S.M.B., see Petersen, M., 43

Nilsen, K.H., see van Walsem, M., 15

Nopoulos, P., 17, 29

Nopoulos, P.C., see Lee, J.K., 57

Nørremølle, A., see Petersen, M., 43

Nørremølle, A., see Nielsen, S.M.B., 34

Ogilvie, C., see Lashwood, A., 59

Øie, L., see Piira, A., 16

Olejniczak, M., see Krzyzosiak, W.J., 53

Olivas, M.R.C., see Calero, V.L.G., 43

Olivas, M.R.C., see Quiñones, H.J.C., 41

Osborne, G.F., see Mielcarek, M., 36

Otani, D.M.V., see Calero, V.L.G., 43

Otani, D.M.V., see Quiñones, H.J.C., 41

Pagonabarraga, J., see Martinez-Horta, S., 45

Pagonabarraga, J., see Perez, J.P., 51
Pakpoor, J., 19

Panegyres, P.K., see Wedderburn, S., 50

Panneck, P., see Nickchen, K., 52

Paola, M.D., 32

Paola, M.D., see Phillips, O., 41

Pardo, A.D., 51

Pardo, A.D., see Paola, M.D., 32

Pardo, A.D., see Phillips, O., 41

Parisi, V., 42

Park, L., see Mielcarek, M., 36

Patassini, S., see Schut, M., 21

Pedroso, J.L., see de Castilhos, R.M., 38

Pedroso, J.L., see de Souza, P.V.S., 39

Pedroso, J.L., see Jardim, L.B., 38

Pepers, B., see Schut, M., 21

Pereira, J.S., see Spitz, M., 28

Pérez, G., see Añez, E.S., 39

Perez, J.P., 51

Perez, J.P., see Martinez-Horta, S., 45

Pérez, N.M., see Posadas, J.J.R., 24, 31

Pestell, C., see Wedderburn, S., 50

Petersen, M., 43

Petersen, M., see Nielsen, S.M.B., 34

Phillips, O., 41

Phillips, O.R., see Paola, M.D., 32

Piemonte, M.E., see Capato, T., 34, 48

Pihet, V., 23

Piira, A., 16

Piira, A., see van Walsem, M., 15

Pineda, L., see Añez, E.S., 39

Pires, R.G.W., see Guimarães, I.M., 40

Polo, C.G., see Posadas, J.J.R., 24, 31

Ponten, H., see Waters, S., 15

Posadas, J.J.R., 24, 31

Poudel, G., see Labuschagne, I., 40

Poudel, G.R., 22, 35

Poudel, G.R., see Domínguez D.J.F., 50

Priller, J., see Gelderblom, H., 18

Priller, J., see Nickchen, K., 52

Prundean, A., see Allain, P., 31

Quarrell, O.W., see McCourt, A.C., 36

Queller, S., see Stout, J.C., 56

Quiñones, H.J.C., 41

Quintero, J.M., see Añez, E.S., 39

Quistorff, B., see Nielsen, S.M.B., 34

Rabinovich-Guilatt, L., 15

Rabinovich-Guilatt, L., 49

Rabinovich-Guilatt, L., see Darpo, B., 26

Rasche, V., see Bornstedt, A., 44 
Rattray, I., 20

Rees, E.M., see Hobbs, N.Z., 54

Reilmann, R., see Gelderblom, H., 18

Reilmann, R., see Landwehrmeyer, B., 30

Rey, V., 30

Ribeiro, F.M., see de Aragão, B.C., 44

Ribeiro, F.M., see Guimarães, I.M., 40

Ribeiro, F.M., see Silva, F.R., 60

Ribeiro, F.M., see Valadão, P.A.C., 19

Rickards, H., see Devlin, R., 55

Rickards, H., see van Duijn, E., 45

Rinaldi, C., see Michele, G.D., 39

Rivadeneyra, J., see Cubo, E., 58

Rivadeneyra, J., see Mariscal, N., 33

Robertson, J., see Mielcarek, M., 36

Robertson, N., see Wild, E., 45

Rodrigues, H.A., see de Aragão, B.C., 44

Rodrigues, M., see Roxburgh, R., 48

Rojas, N., see Aviles, V., 59

Roos, R., see Hart, E., 28

Roos, R., see Heemskerk, A.-W., 33, 56

Roos, R.A., see Hobbs, N.Z., 54

Roos, R.A., see Sathasivam, K., 17

Roos, R.A.C., see Booij, S.J., 60

Roos, R.A.C., see Hubers, A.A.M., 19

Roxburgh, R., 48

Roxburgh, R., see Dysart, J., 58

Russo, A.D., see Saute, J.A.M., 52

Russo, C.V., see Michele, G.D., 39

Sabatini, U., see Dayan, M., 18

Sabatini, U., see Paola, M.D., 32

Sabatini, U., see Phillips, O., 41

Saccà, F., see Michele, G.D., 39

Saft, C., see Gelderblom, H., 18

Salarini, D., see de Castilhos, R.M., 38

Salarini, D., see Jardim, L.B., 38

Salazar, M.R.V., see Calero, V.L.G., 43

Salazar, M.R.V., see Quiñones, H.J.C., 41

Salmon, L., see Domínguez, D.J.F., 28, 50

Salmon, L., see Poudel, G.R., 22, 35

Salvatore, E., see Michele, G.D., 39

Samanamud, D.S.L., see Calero, V.L.G., 43

Samanamud, D.S.L., see Quiñones, H.J.C., 41

Sampaio, C., see Mestre, T., 23

Sampaio, C., see Mestre, T.A., 34

Sanchez-Castaneda, C., see Paola, M.D., 32

Sanchez-Castaneda, C., see Phillips, O., 41

Sanguinetti, A., see Parisi, V., 42

Saraiva-Pereira, M.L., see de Souza, P.V.S., 39
Saraiva-Pereira, M.L., see Souza, A.F.D., 53

Sathasivam, K., 17

Saute, J.A.M., 52

Scahill, R., see Wild, E., 45

Scahill, R.I., see Aylward, E.H., 21

Scahill, R.I., see Hobbs, N.Z., 54

Scherer, C., see Allain, P., 31

Schlagenhauf, F., see Nickchen, K., 52

Schnitzler, A., see Vesper, J., 17

Schuh, A.F.S., see Saute, J.A.M., 52

Schut, M., 21

Sedano, B.P., see Martinez-Horta, S., 45

Sedano, B.P., see Perez, J.P., 51

Seredenina, T., see Mielcarek, M., 36

Silva, C., see Aviles, V., 59

Silva, F.R., 60

Simonsen, A.H., see Vinther-Jensen, T., 58

Sinderu, M.C., see Perez, J.P., 51

Sindreu, M.C., see Martinez-Horta, S., 45

Singh-Bains, M., 56

Sjogren, E., see Heemskerk, A.-W., 56

Smith, D.L., see Mielcarek, M., 36

Smith, D.L., see Sathasivam, K., 17

Smith, E., see Rattray, I., 20

Soares, H., 46

Sonesson, C., see Waters, S., 15

Sorensen, S.A., 22

Sørensen, S.A., see Petersen, M., 43

Sorrentino, P., see Michele, G.D., 39

Souza, A.F.D., 53

Souza, A.F.D., see de Castilhos, R.M., 38

Souza, A.F.D., see Jardim, L.B., 38

Souza, G.N., see Saute, J.A.M., 52

Souza, J.M., see Guimarães, I.M., 40

Spedo, C.T., 24

Spiegelstein, O., see Darpo, B., 26

Spiegelstein, O., see Rabinovich-Guilatt, L., 15, 49

Spitz, M., 28

Sprengelmeyer, R., see Hobbs, N.Z., 54

Spruth, E.J., see Gelderblom, H., 18

Squitieri, F., see Dayan, M., 18

Squitieri, F., see Paola, M.D., 32

Squitieri, F., see Pardo, A.D., 51

Squitieri, F., see Phillips, O., 41

Stout, J.C., 56

Stout, J.C., see Domínguez D.J.F., 28, 50

Stout, J.C., see Labuschagne, I., 40

Stout, J.C., see Poudel, G.R., 22, 35

Süssmuth, S., see Gelderblom, H., 18

Süssmuth, S., see Weydt, P., 36 
Süssmuth, S.D., 26

Sussmuth, S.D., see Hobbs, N.Z., 54

Synek, B., see Singh-Bains, M., 56

Tabrizi, S.J., see Aylward, E.H., 21

Tabrizi, S.J., see Hobbs, N.Z., 54

Tapia, S., see Aviles, V., 59

Taylor, S.M., see Mantovani, S., 23

Tessari, M., see Wild, E., 45

Tian, J., see Zhang, B., 54

Tibben, A., see Booij, S.J., 60

Tibben, A., see van der Meer, L., 47

Tippett, L., see Mehrabi, N., 27

Tippett, L., see Singh-Bains, M., 56

Touller, C., see Mielcarek, M., 36

Träger, U., see Wild, E., 45

Treglia, M., see Grubb, E., 24

Trestman, R.L., see Hennig, B.L., 55

Tricoire, H., see Heidari, R., 46

Tucci, T., see Michele, G.D., 39

Tumas, V., see dos Santos, B.L., 37

Tumas, V., see Spedo, C.T., 24

Valadão, P.A.C., 19

van der Mast, R.C., see Hubers, A.A.M., 19

van der Meer, L., 47

van Duijn, E., 45

van Duijn, E., see Hubers, A.A.M., 19

van Duijn, E., see van der Meer, L., 47

van Ommen, G.-J.B., see Schut, M., 21

van Roon-Mom, W. see Schut, M., 21

van Walsem, M., 15

van Walsem, M., see Piira, A., 16

Vargas, F.R., see de Castilhos, R.M., 38

Vargas, F.R., see Jardim, L.B., 38

Verbist, B., see Heemskerk, A.-W., 33

Verbist, B., see Heemskerk, A.-W., 56

Veriano, C.O., see Valadão, P.A.C., 19

Verny, C., see Allain, P., 31

Vesper, J., 17
Vieira, L.B., see Silva, F.R., 60

Vincent, M.B., see de Cássia Leite Fernandes, R. 59

Vinther-Jensen, T., 58

Wadel, K., see Mielcarek, M., 36

Wagner, P.K., see Leavitt, B.R., 42

Waldvogel, H., 46

Waldvogel, H., see Mehrabi, N., 27

Waldvogel, H., see Schut, M., 21

Waldvogel, H., see Singh-Bains, M., 56

Walker, T., see Rattray, I., 20

Walker, T.A., see Rattray, I., 20

Waters, N., see Waters, S., 15

Waters, S., 15

Wedderburn, S., 50

Weiss, A., see Mielcarek, M., 36

Weiss, A., see Wild, E., 45

Wexler, A., 3

Weydt, P., 36

Weydt, P., see Bornstedt, A., 44

Weydt, P., see Süssmuth, S.D., 26

Wickenberg, A., see Darpo, B., 26

Wickenberg, A., see Landwehrmeyer, B., 30

Wickenberg, A., see Rabinovich-Guilatt, L., 15

Wild, E., 45

Wiltshire, M., see Wedderburn, S., 50

Winge, K., see Vinther-Jensen, T., 58

Winter, L., see Mestre, T., 23

Wojtecki, L., see Vesper, J., 17

Woodruff, T.M., see Mantovani, S., 23

Wu, Q., see Labuschagne, I., 40

Ysabel, M.V.M., see Calero, V.L.G., 43

Ysabel, M.V.M., see Quiñones, H.J.C., 41

Zabala, W., see Añez, E.S., 39

Zakharova, M.N., see Lagarkova, M.A., 16

Zhang, B., 54

Zielonka, D., 49 